\title{
Cosmological structure formation in scalar field dark matter with repulsive self-interaction: The Incredible Shrinking Jeans Mass
}

\author{
Paul R. Shapiro, ${ }^{1 \star}$ Taha Dawoodbhoy, ${ }^{1} \dagger$ and Tanja Rindler-Daller ${ }^{2}+$ \\ ${ }^{1}$ Department of Astronomy and Texas Cosmology Center, The University of Texas at Austin, Austin, TX 78712-1083, USA \\ ${ }^{2}$ Institut für Astrophysik, Universitätssternwarte Wien, University of Vienna, Türkenschanzstr.17, A-1180 Vienna, Austria
}

Accepted XXX. Received YYY; in original form ZZZ

\begin{abstract}
Scalar Field Dark Matter (SFDM) comprised of ultralight $\left(\gtrsim 10^{-22} \mathrm{eV}\right)$ bosons is an alternative to standard, collisionless Cold Dark Matter (CDM) that is CDM-like on large scales but inhibits small-scale structure formation. As a Bose-Einstein condensate, its free-field ("fuzzy") limit (FDM) suppresses structure below the de Broglie wavelength, $\lambda_{\mathrm{deB}}$, creating virialized haloes with central cores of radius $\sim \lambda_{\mathrm{deB}}$, surrounded by CDM-like envelopes, and a halo mass function (HMF) with a sharp cut-off on small scales. With a strong enough repulsive self-interaction (SI), structure is inhibited, instead, below the Thomas-Fermi (TF) radius, $R_{\mathrm{TF}}$ (the size of an SI-pressure-supported ( $\left.n=1\right)$-polytrope), when $R_{\mathrm{TF}}>\lambda_{\mathrm{deB}}$. Previously, we developed tools to describe SFDM dynamics on scales above $\lambda_{\mathrm{deB}}$ and showed that SFDM-TF haloes formed by Jeans-unstable collapse from non-cosmological initial conditions have $R_{\mathrm{TF}}$-sized cores, surrounded by CDM-like envelopes. Revisiting SFDM-TF in the cosmological context, we simulate halo formation by cosmological infall and collapse, and derive its transfer function from linear perturbation theory to produce cosmological initial conditions and predict statistical measures of structure formation, such as the HMF. Since FDM and SFDM-TF transfer functions both have small-scale cut-offs, we can align them to let observational constraints on FDM proxy for SFDM-TF, finding FDM with particle masses $1 \lesssim m /\left(10^{-22} \mathrm{eV} / c^{2}\right) \lesssim 30$ corresponds to SFDM-TF with $10 \gtrsim R_{\mathrm{TF}} /(1 \mathrm{pc}) \gtrsim 1$, favoring sub-galactic (sub-kpc) core-size. The SFDM-TF HMF cuts off gradually, however, leaving more small-mass haloes: its Jeans mass shrinks so fast, scales filtered early can still recover and grow!
\end{abstract}

Key words: cosmology: theory - dark matter - astroparticle physics - large-scale structure of Universe - galaxies: haloes galaxies: formation

\section{INTRODUCTION}

The nature and origin of cosmic dark matter is still unknown. The standard Cold Dark Matter ("CDM") model is actually a place-holder with certain assumed properties - e.g. it behaves during the structure formation era as a nonrelativistic, collisionless gas of particles that couple only weakly to each other and to other components of the Universe, via gravity alone, contributing to the expansion rate of the Universe as a pressure-free component whose mass density, conserved over time, is measured astronomically. A further assumption is made that the CDM particles are born so "cold" - i.e. with such small peculiar motion - that their free-streaming could only have washed out primordial density fluctuations on the tiniest of scales, many orders of magnitude below the scale that affects the formation of galaxies or even their internal structure. With these assumptions, it is possible to work out, within the context of the standard big bang model and inflationary paradigm, how structure formation proceeds from linear-perturbation initial conditions through nonlinear formation of galaxies, clusters and the cosmic web. This model has

\footnotetext{
^ E-mail: shapiro@astro.as.utexas.edu

$\dagger$ E-mail: tahad@astro.as.utexas.edu

† E-mail: tanja.rindler-daller@univie.ac.at
}

successfully accounted for many observational properties of the Universe on large scales, from galaxy formation scales on up. It has, however, been challenged by over-predicting the level of small-scale structure inside galaxies compared to that observed in a variety of systems (see, for instance, Bullock \& Boylan-Kolchin 2017 and references, therein). This may reflect our incomplete knowledge of the impact on galaxy formation of the baryonic component, coupled via gravity to CDM. However, it has also led to a consideration of microscopic properties of CDM that go beyond the standard assumptions described above, in ways that can affect small-scale structure without spoiling its success on larger scales.

The best-studied origin story of CDM involves Weakly Interacting Massive Particles ("WIMPs") with masses in the range $\sim \mathrm{GeV}$ - TeV, predicted by theories of supersymmetry, which form as big bang thermal relics whose abundance is set when their annihilation at a weak-interaction-like rate becomes slower than the Hubble expansion rate. This so-called "standard CDM" - more properly referred to as "WIMP-CDM" - is more specific than the CDM whose generic properties we identified above, with additional observable consequences, and, as such, is more tightly constrained. In particular, it predicts that the same annihilations might lead to their indirect detection from astronomical sources (e.g. by gamma-rays), while their scattering interactions with nucleons might permit their direct 
detection. So far, however, despite considerable effort, there are no confirmed detections, and the allowed range of particle models and parameters that remain viable, consistent with that nondetection, has shrunk (see e.g. Schumann 2019). This has added some urgency to the consideration of alternative models for the origin and microscopic nature of CDM, even apart from the possible conflicts it has of over-predicting small-scale structure on galactic scales. We emphasize this last point at the outset, because it is often missed when evaluating alternatives to WIMP-CDM, by dismissing the alternatives as soon as it appears they are merely "CDM-like" at all scales of interest, i.e. failing to provide the "Deus ex machina" of reducing the apparent over-prediction of small-scale structure by CDM, while retaining all of its successes on larger scales. That is, as long as the origin of CDM is unknown, any microscopic model that is observationally indistinguishable from it is just as successful as any other, until it is disfavored either by further theoretical considerations, or by predictions of additional observables that can distinguish it from the standard model.

One such alternative model of great interest is Scalar Field Dark Matter ("SFDM") comprised of ultralight ( $m \gtrsim 10^{-22} \mathrm{eV} / \mathrm{c}^{2}$ ) bosons, originally suggested because of its novel structure-formation dynamics as a Bose-Einstein condensate and quantum superfluid, described by coupled Schrödinger-Poisson equations. ${ }^{1}$ In the freefield ("fuzzy") limit of SFDM, or fuzzy dark matter ("FDM"), the small-scale structure which we described above for "generic" CDM is suppressed below the de Broglie wavelength, $\lambda_{\mathrm{deB}}$, but resembles CDM on larger scales. Virialized FDM haloes have been shown to have solitonic cores of radius $\sim \lambda_{\mathrm{deB}}$, surrounded by CDM-like envelopes (Schive, Chiueh \& Broadhurst 2014; Schwabe, Niemeyer \& Engels 2016; Mocz et al. 2017). When a strong enough repulsive self-interaction (SI) is also present, structure can be inhibited below a second length scale, $\lambda_{\mathrm{SI}}$, with $\lambda_{\mathrm{SI}}>\lambda_{\mathrm{deB}}-$ referred to as the Thomas-Fermi ("TF") regime. This TF regime of SFDM (henceforth, "SFDM-TF"), assuming a quartic potential for the repulsive SI, has been referred to variously as "BEC-CDM" (Rindler-Daller \& Shapiro 2012, 2014), SFDM (Li, Rindler-Daller \& Shapiro 2014; Li, Shapiro \& Rindler-Daller 2017), "Repulsive Dark Matter" Goodman (2000), or more generally "Fluid Dark Matter" in Peebles (2000). ${ }^{2}$

Structure formation in the TF regime differs significantly from FDM. In Dawoodbhoy, Shapiro \& Rindler-Daller (2021) (henceforth Paper I), we discussed the nonlinear dynamics and internal structure of haloes that form from gravitational instability in the TF regime. Here we revisit this in the context of halo and large-scale structure

\footnotetext{
1 The field of SFDM study has an extensive literature, well-beyond our ability to cite it here. A few early references not otherwise cited in this paper include Sin (1994); Lee \& Koh (1996); Matos, Guzmán \& UreñaLópez (2000); Guzmán \& Ureña-López (2004); Matos, Vázquez-González \& Magaña (2009); Suárez \& Chavanis (2017); Desjacques, Kehagias \& Riotto (2018). Some reviews with additional references therein include Suárez, Robles \& Matos (2014); Rindler-Daller \& Shapiro (2014); Marsh (2016).

2 Note that SFDM-TF should not be confused with another CDM alternative known as self-interacting dark matter (SIDM). The latter behaves as a collection of point-particles undergoing elastic scatterings, which amounts to adding 2-body elastic collisions to the standard, collisionless gravitational dynamics of CDM, while the former is a BEC, best thought of as a classical field evolving under a continuous self-interaction potential (along with the gravitational potential). The two models are phenomenologically distinct, as is evident by the rôle SI plays in their respective non-relativistic fluid equations. In the SFDM-TF model (as discussed in \$2.4), SI enters as a potential or pressure term in the momentum equation (equation 27), whereas in the SIDM model, SI enters as a heat conduction term in the energy equation (Balberg, Shapiro \& Inagaki 2002; Ahn \& Shapiro 2005).
}

formation from cosmological perturbations, including observational constraints. As this is a companion paper ("Paper II"), we will sometimes refer the reader to Paper I for details, for the sake of brevity and to avoid redundancy.

In Paper I, we developed equations and tools to calculate the dynamical evolution of inhomogeneity in response to gravitational instability in this model, starting from the nonlinear Schrödinger equation ("NLSE"). A key development was to take full account of both quantum pressure (associated with inhomogeneity on the tiny scale of the de Broglie wavelength) and SI, based upon a reformulation of the NLSE, smoothed on scales larger than the de Broglie scale. In that case, the NLSE reduced to the collisionless Boltzmann equation that describes CDM, except with the SI potential added to the usual gravitational potential. From this, a fluid approximation for SFDM-TF was derived, which added a new pressure force for SI to equations we previously derived for CDM. This enabled us to describe the scalar field by the familiar hydrodynamical conservation equations for a $\gamma=5 / 3$ ideal gas, with an ideal gas pressure, $P_{\sigma}$, associated with particle velocity dispersion (which accounts for the large-scale effects of quantum pressure), and with a second pressure, $P_{\text {SI }}$, which accounts for the repulsive SI force. This fluid approximation allowed us to evolve SFDM-TF computationally in spherical-1D with the very high resolution and dynamic range which are required (but not currently possible) for full 3D simulations of SFDM-TF. With this tool, we solved the Newtonian problem of virialized halo formation by the nonlinear collapse of a Jeans-unstable perturbation, from non-cosmological (i.e. static) initial conditions, for the first time. These simulations in Paper I showed that haloes formed in this way have core-envelope density profiles, with flattened cores of size $R_{\mathrm{TF}}$ - the radius of the ( $\left.n=1\right)$-polytrope that forms for a self-gravitating fluid in hydrostatic equilibrium when supported by SI pressure alone, referred to as the Thomas-Fermi radius - surrounded by an envelope just like the one that formed when CDM dynamics was simulated, instead, from the same initial conditions. This explained why, in the case of FDM, when haloes form in 3D simulations (which are possible for FDM, where the de Broglie wavelength is large enough to be fully resolvable, unlike SFDM-TF, for which de Broglie wavelengths are unresolvably small), density profiles are also CDM-like outside their solitonic cores (of size close to the de Broglie wavelength).

In Paper I, we showed how SFDM-TF haloes with the right value of $R_{\mathrm{TF}}$ could resolve the cusp-core and "too-big-to-fail" (TBTF) problems of CDM, without suffering from the problem identified for FDM by Robles, Bullock \& Boylan-Kolchin (2019) in which the solitonic cores of larger-mass FDM haloes were too dense to be consistent with the rotation curves of larger-mass galaxies, if the FDM particle mass were tuned to $m \simeq 10^{-22} \mathrm{eV} / c^{2}$ to match observations of smaller-mass galaxies. We found that $R_{\mathrm{TF}} \gtrsim 1 \mathrm{kpc}$ is required. It was left to Paper II, here, to determine if such a value of $R_{\mathrm{TF}}$ was consistent with the cosmological formation of those haloes.

In what follows, we study the cosmological context for structure formation in the SFDM-TF model, both by simulating individual halo formation cosmologically, and by deriving the initial conditions for Gaussian-random primordial fluctuations from linear perturbation theory. In $\S 2$, we present the model and basic equations we use to study SFDM-TF cosmologically. In $\S 3$, we apply these equations to simulate individual halo formation by spherical infall and collapse in a cosmologically expanding SFDM-TF universe. The overall scenario for cosmic structure in the SFDM-TF model is developed in $\S 4$, including linear perturbation theory to derive the transfer function and power spectrum, as well as their application to statistical measures of structure formation like the halo mass function. We apply 
the same approach to the FDM model, as well, for comparison. In $\S 4.7$, we present the results of this linear perturbation theory and use observational constraints on the FDM model, fully parameterized by its particle mass $m$, as a proxy to derive constraints on the SFDM-TF model, parameterized by $R_{\mathrm{TF}}$. Our summary and conclusions are presented in $\$ 5$.

\section{THE MODEL AND BASIC EQUATIONS}

\subsection{The fully relativistic description}

We model SFDM as a classical, massive, complex scalar field $\phi$ with a quartic, repulsive self-interaction (SI), which obeys the KleinGordon equation

$\square \phi+\frac{m^{2} c^{2}}{\hbar^{2}} \phi+\frac{2 g}{\hbar^{2}}|\phi|^{2} \phi=0$

where $\square \equiv \nabla_{\mu} \nabla^{\mu}$ is the d'Alembertian for a general 4D spacetime metric, $m$ is the boson mass, and $g$ is the SI coupling strength. This equation is coupled to the Einstein equations, with a metric tensor given by the Friedmann-Lemaitre-Robertson-Walker metric for a homogeneous and isotropic background universe, perturbed by primordial fluctuations. The scalar field contributes terms to the stress-energy tensor in the Einstein equations, which contain the field and its derivatives.

As we described in Li et al. (2014, 2017), the evolution of the homogeneous background scalar field corresponds to that of a perfect fluid. However, the scalar field oscillates with an angular frequency which eventually exceeds the Hubble expansion rate, when

$\omega \simeq \frac{m c^{2}}{\hbar} \sqrt{1+\frac{2 g}{m^{2} c^{2}}|\phi|^{2}} \gg H$

(see equation 21 in Li et al. 2014), where the square of the modulus of the field, $|\phi|^{2}$, is, at late times, related to the SFDM mass density by $\rho_{b}=\left\langle|\phi|^{2}\right\rangle=\rho_{b}(a=0) a^{-3}$ (the subscript " $b$ " refers to background), and the Hubble parameter $H(t)=(d a / d t) / a$ with scale factor $a=a(t)$ depending on cosmic time $t$. Prior to this rapid oscillation epoch, the field evolution was shown to start from a kinetic-energy-dominated, stiff, relativistic phase during the slowoscillation period when $\omega \ll H$, but eventually to transition over time to a radiation-like, still relativistic phase during the rapid oscillation period, when the SI term in equation (1) dominates the quadratic mass term. Finally, at later times, when the mass term dominates, there is a transition to a nonrelativistic, matter-like phase. More precisely, as a perfect fluid, the field can be characterized by an equation of state parameter $w$ defined as the ratio of pressure to energy density, which varies over time to reflect these phases of evolution. Initially, $w=1$ (stiff phase), which must end prior to the epoch of big bang nucleosynthesis $(\mathrm{BBN})$, in order not to spoil the agreement between predictions of the standard model involving primordial nucleosynthesis in a radiation-dominated (RD) universe with the observed relic abundances of the light elements. Eventually, when the field oscillates much more rapidly than the Hubble expansion rate, we can average the pressure $P$ and energy-density $\varepsilon$ of the field over the oscillation period and take the ratio of these average values to give the average value of the equation of state parameter $w$, according to the following (which is equation 26 in Li et al. 2014):

$$
\langle w\rangle \equiv \frac{\langle P\rangle}{\langle\varepsilon\rangle}=\frac{1}{3}\left(\frac{1}{1+\frac{2 m^{2} c^{2}}{3 g\left\langle|\phi|^{2}\right\rangle}}\right)
$$

When the SI term dominates during this phase, $\langle w\rangle \simeq 1 / 3$ (radiationlike), but at late times, before the universe made its transition from radiation- to matter-dominated (MD), the mass term must come to dominate and $\langle w\rangle \simeq 0$ thereafter, rendering SFDM matter-like, as it should be in order to account for the MD era. ${ }^{3}$

\subsection{Matter-like regime and the Newtonian approximation}

In this latter $(\langle w\rangle \simeq 0)$ regime, in the limit of weak-field (i.e. Newtonian) gravity and even in the presence of inhomogeneities, as long as they are on scales well-within the horizon (i.e. $\ll c / H$ ), we can adopt the Newtonian approximation in which gravity obeys the Poisson equation. The equation of motion of the scalar field in this nonrelativistic regime then simplifies to the nonlinear Schrödinger equation (NLSE) in an expanding background, by factorizing the field into a slowly-varying "mean" field $\psi$ and an oscillatory component, as follows: ${ }^{4}$

$\phi=\psi e^{-i m c^{2} t / \hbar} \quad\left|\partial_{t} \psi\right| \ll\left|\frac{m c^{2}}{\hbar} \psi\right|$

In terms of the comoving coordinate $x$, defined with respect to the proper coordinate $\boldsymbol{r}$ such that

$\boldsymbol{x}=\boldsymbol{r} / a$

$\nabla \equiv \nabla_{\boldsymbol{x}}=a \nabla_{\boldsymbol{r}}$

and

$\partial_{t} \equiv\left(\partial_{t}\right)_{\boldsymbol{x}}=\left(\partial_{t}\right)_{\boldsymbol{r}}+H \boldsymbol{r} \cdot \nabla_{\boldsymbol{r}}$

where $\left(\partial_{t}\right)_{\boldsymbol{x}}(\boldsymbol{r})$ represents the partial time derivative at fixed comoving (proper) coordinate $\boldsymbol{x}(\boldsymbol{r})$, the NLSE and Poisson equations are then

$i \hbar\left(\partial_{t}+\frac{3}{2} H\right) \psi=\left(-\frac{\hbar^{2}}{2 m a^{2}} \nabla^{2}+\frac{g}{m}|\psi|^{2}+m \Phi\right) \psi$

and

$\nabla^{2} \Phi=4 \pi G a^{2}\left(\rho-\rho_{b}\right)$

with

$\rho=|\psi|^{2}$

where $\Phi$ is the peculiar Newtonian gravitational potential, $\rho$ is the mass density of the scalar field, and $\rho_{b}=\rho_{b}(a=0) a^{-3}$ is the homogeneous background value. ${ }^{5}$

3 This description above from Li et al. (2014, 2017), of the evolution of the EOS of the homogeneous scalar field, from stiff, relativistic $(w=1)$ at early times, before the rapid-oscillation phase, to radiation-like $(\langle w\rangle \simeq 1 / 3)$ when rapid oscillation begins and SI dominates, and finally to nonrelativistic, matter-like $(\langle w\rangle \simeq 0)$, was derived for a complex scalar field. However, we are only interested here in the rapid-oscillation phase, during which the EOS is radiation-like when the SI term dominates over the mass term in the Lagrangian, then transitions to matter-like when the latter term dominates. As such, what happens before the rapid-oscillation phase begins does not affect our calculations here. In that case, we do not expect our calculations to depend upon whether the field is complex or real, since the description above of the EOS evolution during the rapid-oscillation phase applies equally well to the real and complex cases.

${ }^{4}$ If $\phi$ is a real field, then one should add to the right side of equation (4) the complex conjugate, i.e. $\phi=\psi e^{-i m c^{2} t / \hbar}+\psi^{*} e^{i m c^{2} t / \hbar}$, where $\psi$ is still a complex field.

5 We shall generally assume here, unless otherwise stated, that the nonrelativistic matter density is dominated by the scalar field dark matter density, so we do not need to keep separate track of other nonrelativistic mass components like the baryons. 
These equations were presented in Paper I in the Newtonian limit in the absence of cosmological expansion. The difference here is that we represent derivatives and spatial coordinates in the comoving frame, which introduces the homogeneous expansion term involving Hubble parameter $H(t)$, and the peculiar gravitational potential $\Phi$. If the scale factor were constant, $a(t)=1$, we would, of course, recover the equations of Paper I.

As we discussed in detail in Paper I, there is a fully equivalent formulation of this nonrelativistic NLSE as quantum hydrodynamics equations ("QHD"). In particular, the NLSE can be transformed into a pair of hydrodynamical mass and momentum conservation equations via the Madelung transformation, in which the field is expressed in polar form as

$\psi=|\psi| e^{i S}$

and the conservation of current density allows us to identify the peculiar bulk fluid velocity in the current flux density as

$\boldsymbol{v}=\frac{\hbar}{m a} \nabla S$

This leads to

$\partial_{t} \rho+3 H \rho+\frac{1}{a} \nabla \cdot(\rho \boldsymbol{v})=0$

and

$\partial_{t} \boldsymbol{v}+H \boldsymbol{v}+\frac{1}{a}(\boldsymbol{v} \cdot \nabla) \boldsymbol{v}+\frac{1}{a} \nabla\left(Q+V_{\mathrm{SI}}+\Phi\right)=0$

where the "quantum potential" $Q$ and self-interaction potential $V_{\mathrm{SI}}$ are given by

$Q=-\frac{\hbar^{2}}{2 m^{2} a^{2}} \frac{\nabla^{2} \sqrt{\rho}}{\sqrt{\rho}}$

and

$V_{\mathrm{SI}}=\frac{g \rho}{m^{2}}$

These may also be written as pressure terms:

$\nabla Q=\frac{1}{\rho} \nabla \cdot \boldsymbol{\Pi}$

where $\boldsymbol{\Pi}$ is the momentum flux density tensor, also known as the "quantum pressure" tensor, given by

$\Pi_{i j}=\left(\frac{\hbar}{2 m a}\right)^{2}\left(\frac{1}{\rho} \frac{\partial \rho}{\partial x_{i}} \frac{\partial \rho}{\partial x_{j}}-\frac{\partial^{2} \rho}{\partial x_{i} \partial x_{j}}\right)$

and

$\nabla V_{\mathrm{SI}}=\frac{1}{\rho} \nabla P_{\mathrm{SI}}$

where $P_{\mathrm{SI}}$ is the self-interaction (SI) pressure, given by

$P_{\text {SI }}=\frac{g \rho^{2}}{2 m^{2}}$

In the matter-like phase when $\langle w\rangle \simeq 0$, the parameter $g / \mathrm{m}^{2}$ is related to the radius $R_{\mathrm{TF}}$ of the ( $\left.n=1\right)$-polytrope which results when gravity balances SI pressure in hydrostatic equilibrium, sometimes referred to as the Thomas-Fermi (TF) radius, according to

$R_{\mathrm{TF}}=\pi \sqrt{\frac{g}{4 \pi G m^{2}}} \simeq 1.1 \mathrm{kpc}\left(\frac{g /\left(m c^{2}\right)^{2}}{2 \times 10^{-18} \mathrm{eV}^{-1} \mathrm{~cm}^{3}}\right)^{1 / 2}$

\subsection{The TF regime and the limit of small de Broglie wavelength}

As discussed in Paper I, the same QHD equations can also by derived by a reformulation of the NLSE in phase space, involving momentum moments of a quantum phase space distribution function. In one version of that approach, involving a smoothed phase space distribution, we showed that when the smoothing scale is much larger than the de Broglie wavelength $\lambda_{\mathrm{deB}}$, the NLSE is equivalent to the Collisionless Boltzmann Equation ("CBE"), except with the gravitational potential term replaced by the sum of the gravitational and SI potentials. This reduction of the NLSE to the CBE in the small- $\lambda_{\mathrm{deB}}$ limit, for the free-field case without the modification to account for SI introduced in Paper I, was previously discussed by Mocz et al. (2018), in regards to the FDM model, following earlier work by Widrow \& Kaiser (1993) and Skodje, Rohrs \& Vanbuskirk (1989). Since the description of standard CDM as a gas of point-particles which are nonrelativistic, noninteracting (except gravitationally), and collisionless (i.e. the 2-body gravitational relaxation time is much longer than the lifetime of the system) is consistent with the CBE, this explains why FDM behaves just like CDM, dynamically, on scales much larger than $\lambda_{\mathrm{deB}}$, i.e. when viewed in this coarse-grained way that does not resolve substructure on scales smaller than $\lambda_{\mathrm{deB}}$. For example, numerical simulations of the NLSE in the FDM limit (no SI) show that, when haloes form gravitationally and virialize, they have a core-envelope structure in which a solitonic core of size $\sim \lambda_{\mathrm{deB}}$ (with a centrally-flattened density profile like the ground-state, static equilibrium solution of the 1D, spherical NLSE) is surrounded by an envelope with a profile like that which would have occurred in standard CDM. With strong enough SI to be in the TF regime, however, we showed in Paper I that the solitonic core would be replaced by a core, much larger than $\lambda_{\mathrm{deB}}$ (the latter is arbitrarily small in this TF regime), supported instead by SI pressure, while outside this SI-supported core, a CDM-like envelope would still be expected. However, to solve the latter problem in this TF regime, by the same kind of 3D simulations as were done for FDM, is much more difficult, since it would require fully resolving length scales well-below $\lambda_{\mathrm{deB}}$ in order to produce the correct CDM-like behavior outside the core. In the TF regime, $\lambda_{\mathrm{deB}}$ is much smaller than the size of the SI-smoothed halo cores, which are, themselves, much smaller than the haloes, and even smaller relative to the large-scale structure from which they formed, so the required resolution and dynamic range becomes prohibitively expensive. We solved this problem by finding an approximate fluid description for SFDM-TF, as described below.

\subsection{The fluid approximation for SFDM-TF}

In Paper I, we were tasked with calculating the dynamical formation of virialized haloes in SFDM-TF by evolving the scalar field from a nearly-uniform, linearly-perturbed, initial condition through many orders of magnitude of gravitational collapse, all the while keeping track of the dominant dynamical effects involving quantum pressure, which result from inhomogeneities on the scale of the unresolvablysmall $\lambda_{\mathrm{deB}}$. The fact discussed there (noted above in $\S 2.3$ ), that, in the small- $\lambda_{\mathrm{deB}}$ limit, the NLSE for SFDM-TF can be shown to reduce to the same $\mathrm{CBE}$ as for CDM, except modified by adding the SI potential to that for gravity, suggests that, in principle, an SI-modified N-body method might be developed to solve this problem, if we were willing to forego knowledge of the detailed substructure on the scale of $\lambda_{\mathrm{deB}}$, while still accounting for the effects of quantum pressure on larger scales in a coarse-grained way. However, such an N-body method would still require an extremely large dynamic range to simulate this in $3 \mathrm{D}$, in order to resolve the small-scale suppression effects of SI 
pressure on scales below $R_{\mathrm{TF}}$, even in this limit in which the (much smaller) de Broglie scale is left unresolved - a high computational barrier. Instead, we avoided the complicated challenge of such a grand computational solution by closing the infinite hierarchy of the momentum moments of the CBE by following the approach developed for standard CDM dynamics by Ahn \& Shapiro (2005).

Ahn \& Shapiro (2005) reduced the CBE equation for CDM to a set of equations identical to the hydrodynamical equations of conservation of mass, momentum, and energy for an ideal gas with ratio of specific heats $\gamma=5 / 3$, in $1 \mathrm{D}$, spherical symmetry, by assuming the velocity distribution is skewless and isotropic in the frame of bulk motion. As discussed there, these are assumptions that approximate the results of CDM N-body simulations reasonably well, for the virialized regions identified as haloes. ${ }^{6}$ In the case of SFDM-TF, however, as we showed in Paper I, we must replace the gravitational potential in the CDM case of Ahn \& Shapiro (2005) by the sum of the gravitational and SI potentials. As a result, a new kind of pressure force appears in the momentum equation, in addition to the ideal gas pressure, namely the SI pressure force, involving the gradient of the SI pressure given above in equations (19) and (20).

The resulting spherically-symmetric fluid equations for SFDM-TF are presented below in proper coordinates, in Eulerian form:

$$
\begin{aligned}
\text { [continuity] } & \frac{\partial \rho}{\partial t}+\frac{1}{r^{2}} \frac{\partial\left(r^{2} \rho v\right)}{\partial r}=0 \\
\text { [momentum] } & \frac{\partial v}{\partial t}+v \frac{\partial v}{\partial r}+\frac{1}{\rho} \frac{\partial}{\partial r}\left(P_{\sigma}+P_{\mathrm{SI}}\right)+\frac{\partial \Phi}{\partial r}=0 \\
\text { [energy] } & \frac{\partial}{\partial t}\left(\frac{3 P_{\sigma}}{2 \rho}\right)+v \frac{\partial}{\partial r}\left(\frac{3 P_{\sigma}}{2 \rho}\right)+\frac{P_{\sigma}}{\rho r^{2}} \frac{\partial\left(r^{2} v\right)}{\partial r}=0
\end{aligned}
$$

where $P_{\sigma}=\rho \sigma^{2}$ is an isotropic pressure associated with the velocity dispersion $\sigma$ obtained from velocity moments of the phase space distribution function. This pressure, which behaves like the pressure of an ideal gas with ratio of specific heats $\gamma=5 / 3$ and, hence, contributes a pressure-gradient force to the momentum equation, while changing its specific internal energy $u \equiv\left(P_{\sigma} / \rho\right) /(\gamma-1)$ to account for $P_{\sigma} d V$-work, thereby accounts for the large-scale effects of the quantum pressure tensor ( $\boldsymbol{\Pi})$, as discussed in Paper I. If we define the mass interior to radius $r$ as $M_{r}$, according to

$M_{r} \equiv M(\leq r)=\int_{0}^{r} 4 \pi\left(r^{\prime}\right)^{2} \rho d r^{\prime}$

we can rewrite equations (22) - (24) in Lagrangian form, though still in proper coordinates, as follows:

$$
\begin{aligned}
\text { [continuity] } & \frac{D \rho}{D t}=-\frac{\rho}{r^{2}} \frac{\partial\left(r^{2} v\right)}{\partial r} \\
\text { [momentum] } & \frac{D v}{D t}=-4 \pi r^{2} \frac{\partial}{\partial M_{r}}\left(P_{\sigma}+P_{\mathrm{SI}}\right)-\frac{G M_{r}}{r^{2}} \\
\text { [energy] } & \frac{D}{D t}\left(\frac{3 P_{\sigma}}{2 \rho}\right)=-P_{\sigma} \frac{D(1 / \rho)}{D t}
\end{aligned}
$$

where the Lagrangian, or total, time derivative is given by

$\frac{D}{D t} \equiv \frac{\partial}{\partial t}+v \frac{\partial}{\partial r}$

In Paper I, we also stated the adiabatic shock jump conditions that accompany these differential conservation equations when there are discontinuities present. The reader is referred to Paper I for those.

\footnotetext{
${ }^{6}$ Outside of virialized regions, infall is highly supersonic; the velocity of bulk peculiar motions greatly exceeds that associated with the velocity dispersion due to random motions in that frame. Hence, these assumptions make no appreciable difference in the dynamics outside virialized regions.
}

\section{HALO FORMATION BY COSMOLOGICAL INFALL: SPHERICAL COLLAPSE AND VIRIALIZATION}

\subsection{The non-cosmological case: nonlinear Jeans instability from a static initial condition}

In Paper I, we solved the equations above to follow the spherical collapse of a Jeans-unstable linear perturbation in a static background. We simulated the nonlinear evolution of a region containing a mass much larger than the Jeans mass, initially at rest, from a uniform background density, perturbed by a spherically-symmetric perturbation that is slightly concentrated toward the center. As it was gravitationally unstable, the mass collapsed, first at the center, until the rising SI pressure inside the central region of radius $\simeq R_{\mathrm{TF}}$ halted the gravitational collapse of the innermost mass shells, and a strong accretion shock formed there to decelerate other incoming shells. As more mass collapsed and would have, if entirely pressure-free, reached the origin in a finite time, with shells originating at successively larger radii predicted to reach the origin at successively later times, each shell, instead, encountered the shock at a finite radius and was halted by it. Outside this accretion shock, the infall was highly supersonic and the ideal gas "temperature" (i.e. the velocity dispersion in momentum space, as measured in the frame of bulk radial motion) was very small, so the ideal gas pressure associated with it was too small to halt the collapse. The SI pressure was also low there, so it, too, was unable to decelerate the infall. When a mass shell crossed the accretion shock, however, its "temperature" and ideal gas pressure jumped by orders of magnitude, while its density jumped only by a factor of four (i.e. the value for a strong, adiabatic shock jump in an ideal gas with a ratio of specific heats $\gamma=5 / 3$ ). As a result, the post-shock gas was dominated by ideal gas pressure, high enough to bring the mass shell to an approximate rest in hydrostatic equilibrium ("HSE"). In this post-shock HSE, ideal gas pressure balanced gravity everywhere inside the shock, except inside $\sim R_{\mathrm{TF}}$, where it was SI pressure that provided the support, instead, and the ideal gas pressure was unimportant. Over time, more mass shells reached the accretion shock, which gradually moved outward, encompassing an ever-increasing mass. As new mass shells passed through the shock and steadily added their mass to that of the previously-shocked shells, they extended the radius of the post-shock region in hydrostatic equilibrium as the shock moved outward. The structure interior to the post-shock region corresponds to the "virialized halo" in cosmological simulations.

In Paper I, we compared the outcomes of two simulations from the same initial conditions, one that included the SI pressure for SFDMTF and one that did not, so the latter was entirely "CDM-like", therefore. Since the initial conditions for both of these simulations was that for a Jeans-unstable density perturbation on a static homogeneous background, the collapse that ensued left a post-shock density profile in the CDM-like case (i.e. with no SI) with a radial dependence $\rho \propto r^{-12 / 7}$. As we explained there, this slope results when cold, infalling gas with a density profile with this slope the same as reported in earlier work on infall from instability in a spherical, Jeans-unstable gas cloud (e.g. Penston 1969) - encounters the strong accretion shock. The shock jump converts the kinetic energy of this cold, supersonic infall into ideal-gas internal energy, with enough post-shock gas pressure to establish hydrostatic equilibrium. According to the strong, adiabatic shock jump conditions, the density must also increase, jumping by a constant factor of 4 (for $\gamma=5 / 3$ ). As a result, the static post-shock density profile inside the shock must have the same power-law profile as the pre-shock infall, outside the shock, namely, $\rho \propto r^{-12 / 7}$. The SFDM-TF solution was shown to be identical to this CDM solution everywhere outside of the 
SI-pressure-supported core of radius equal to $\sim R_{\mathrm{TF}}$. Inside this radius, however, the density profile was flattened, following that of the $(n=1)$-polytropic profile that would result if SI pressure alone supported the scalar field against its own self-gravity. Its central density was set by the condition that it conserved the mass that would have been there inside that radius if the CDM-like profile had continued onward to the origin, uninterrupted by SI pressure.

In short, the generic structure of the haloes that formed as the nonlinear outcome of Jeans instability in SFDM-TF, therefore, could be described simply as the core-envelope structure in which a flattened, SI-polytropic core of radius $\sim R_{\mathrm{TF}}$ was surrounded by the same CDM-like envelope it would have had if there were no SI pressure, and the mass inside the polytropic core radius was the same as it would have been in that CDM-like profile in the absence of SI. As such, this also served to compare the SFDM-TF profile with the corresponding FDM (i.e. "free-field" limit) profile that would have resulted from the same initial conditions if $\lambda_{\mathrm{deB}}$ is small, since the latter was shown in Paper I to be identical to the CDM profile, when smoothed on scales large compared with $\lambda_{\mathrm{deB}}$.

We note that, although the description above applies to the entire post-shock region at a given time, we can make our description apply, as well, to cosmological haloes, which are customarily identified as the "virialized regions" bounded by a "virial radius" inside of which the average mass density is equal to some fixed constant multiple of the unperturbed background density of the universe at that cosmic time. In the Jeans-unstable collapse calculations in Paper I from static initial conditions, there is not a cosmological background density to relate to the overdensity inside some radius within the post-shock region, but we are still free to adopt an overdensity like the conventional definition, relative to the unperturbed background density, in order to determine the radius (within the post-shock sphere) which we can call the "virial radius".

\subsection{The cosmological case: spherical infall in the expanding universe}

The description above is a guide to what we can expect below, when we re-consider the nonlinear Jeans instability in SFDM-TF here, to place it in the proper cosmological context of the expanding universe in the MD era, i.e. after matter-radiation equality, $a>a_{\text {eq }}$. To do this, we will solve the same equations, as written in proper coordinates, but now with cosmological initial and boundary conditions, ${ }^{7}$ to follow the spherical collapse of a linear perturbation in the expanding universe. This is fully cosmological, for structure formation on scales that are well within the horizon when we evolve them. As we shall see, the novel effect of cosmological expansion is to allow mass shells that were initially inside the characteristic radius of the effects of SI pressure to expand outward for a time, carrying them beyond the reach of this repulsive force, and then to fall back again, to re-encounter it after turn-around and collapse. An imprint of this initial encounter remains, however, and has important consequences for their final resting place.

7 Although our dynamical equations (22) - (24) do not appear to be cosmological, for a spherically-symmetric distribution of matter in the nonrelativistic and weak gravitational field limits, Birkhoff's Theorem implies that Newtonian gravity is sufficient for describing cosmological dynamics. Therefore, our cosmology is taken care of by the Newtonian potential and the inclusion of Hubble flow (and its perturbation) in the initial velocity.

\subsubsection{CDM-like initial conditions: the infall rate that makes an NFW profile}

Our goal in this paper ("Paper II") is to generalize the noncosmological results in Paper I to investigate the cosmological formation of SFDM-TF haloes. Our first approach in this direction follows the tradition of previous study of structure formation in the free-field limit of the SFDM model (FDM), in which the first cosmological simulations assumed scalar field dynamics but initial conditions like those for the CDM model. In particular, we will simulate the formation of individual haloes from the same initial conditions for both CDM and SFDM-TF, in order to learn what difference results from scalar field dynamics with repulsive SI, as opposed to the collisionless dynamics of CDM.

It is useful to begin by summarizing what is known about the CDM case and why it is that we have confidence in the $1 \mathrm{D}$, spherical collapse calculations we propose to use to study the contrast between CDM and SFDM-TF halo dynamics. In Alvarez, Ahn \& Shapiro (2003), Shapiro et al. (2004), Ahn \& Shapiro (2005) and Shapiro et al. (2006), we showed that the 1D, spherical infall model involving the same fluid approximation as used here and in Paper I, derived by Ahn \& Shapiro (2005) from the collisionless Boltzmann equation, can explain the universal structure of haloes in N-body simulations of nonlinear structure formation from Gaussian-random-noise density fluctuations in the CDM universe. As a first step, Ahn \& Shapiro (2005) applied this fluid approximation for CDM to derive an analytical similarity solution for halo formation from spherical infall. As described below, this produced a density profile inside the accretion shock in this solution which was a surprisingly good match to various empirical formulae developed to fit density profiles of CDM haloes in N-body simulations, e.g. the NFW profile Navarro, Frenk \& White (1997). However, while the agreement was impressive at a given snapshot of time, the N-body results showed that, if captured at different times in the mass assembly history ("MAH") of the same halo, the shape of its profile would evolve, while that of a self-similar profile does not. As Ahn \& Shapiro (2005) pointed out, this reflected the fact that infall rates of individual haloes derived by fitting the Lagrangian MAH of haloes in CDM N-body simulations vary over the lifetime of each halo - rapid early, then slowing down late - while the rate in their self-similar solution $(d \ln M / d \ln a=6)$ is fixed, corresponding to the early, rapid-infall phase of the N-body results. To better match the non-self-similar shape evolution of the $\mathrm{N}$-body haloes, in Alvarez et al. (2003) we replaced the self-similar initial perturbation in Ahn \& Shapiro (2005) by a non-self-similar spherically-symmetric perturbation profile calculated to make a timevarying infall rate that matched the MAH of CDM N-body haloes. Solving the 1D fluid approximation equations for CDM in this case required a numerical hydrodynamics code. The haloes that resulted were shown to be in excellent agreement with the universal average behavior of the haloes in N-body simulations. Since this earlier work, also summarized in Shapiro et al. (2004) and updated in Shapiro et al. (2006), is central to our adoption of CDM-like initial conditions here to demonstrate the difference between SFDM and CDM dynamics on cosmological halo formation, we describe it in more detail below.

\subsubsection{CDM haloes from self-similar spherical infall: $\epsilon=1 / 6$}

In an Einstein-de Sitter universe, a scale-free, spherically-symmetric linear perturbation $\delta M / M \propto M^{-\epsilon}$ leads to self-similar halo formation around the density peak at the center. According to the theory of halo formation from peaks in the density field which result from Gaussian-random-noise initial density fluctuations, the average den- 
sity profile around such peaks can be related to the shape of the fluctuation power spectrum (Hoffman \& Shaham 1985; Bardeen et al. 1986). These density peaks in the linear regime are the progenitors of nonlinear structures like haloes. It is useful to describe such peaks in terms of the RMS mass fluctuation, $\sigma_{\mathrm{M}}$, of the overdensity field smoothed on some mass scale $M$. The average linear overdensity profiles around peaks of overdensity $v \sigma_{\mathrm{M}}$ are triaxial, in general, but approach spherical symmetry as $v$ increases (e.g. for $v \geq 3$ ), with cumulative overdensity $\Delta_{0}$ inside radius $r$, given by $\Delta_{0} \propto r^{-(n+3)}$, where $n$ is the effective index of the (transferred) power spectrum $P(k)$ if approximated as a power-law $P(k) \propto k^{n}$ at the wavenumber $k$ that corresponds to that halo mass. In terms of fractional mass perturbation, $\Delta_{0}=\delta M / M \propto M^{-(n+3) / 3}$, so a power-law $P(k) \propto k^{n}$ leads naturally to a scale-free initial condition with $\epsilon=(n+3) / 3$. While the actual transferred $P(k)$ for CDM is not a power-law, it is possible to use the actual shape to derive an effective power-law index which varies with halo mass $M$ as a good approximation. Ahn \& Shapiro (2005) calculated this $n_{\text {eff }}$ for the current $\Lambda$ CDM universe, for the full range of halo masses from $10^{3}$ to $10^{15} \mathrm{M}_{\odot}$, showing that galactic haloes are well-approximated by $n=-2.5$, since $n \cong-2.5 \pm 0.2$ for $M$ in the range from $10^{3} \mathrm{M}_{\odot}$ to $10^{11} \mathrm{M}_{\odot}$. For haloes of galactic mass, formation by spherical infall from a self-similar initial perturbation with $\epsilon=1 / 6$ is therefore a reasonable first approximation (for the MD era, for which the universe is well-approximated as Einstein-de Sitter).

The resulting self-similar density profile agreed with that for the NFW profile to within $10 \%$, at radii $r \lesssim 0.6 R_{200}$, for a best-fitting concentration parameter of 3 , including a density cusp at small radii with a logarithmic slope $\approx-1.27$ (for $4 \times 10^{-3}<r / R_{200}<1.4 \times$ $10^{-2}$ ), where $R_{200}$ is the radius within which the average density is 200 times the cosmic mean density. Furthermore, as we showed in Shapiro et al. (2006), the analytical similarity solution yields a profile for the quantity $\rho / \sigma_{V}^{3}$, sometimes referred to as the "phasespace density", which is best-fit by a power-law $\rho / \sigma_{V}^{3} \propto r^{-1.91}$, in close agreement at all times with the universal profile reported in the literature for CDM N-body simulation results cited there. However, the radius of the accretion shock that bounds the virialized region in this solution is somewhat smaller than $R_{200}$, located instead at the radius which encloses an average overdensity of 564 times this mean density, at $R_{564} \simeq 0.6 R_{200}$. This suggests that the rapid early phase of MAH in the N-body results, which deposits the mass of the density profile at inner radii that remains there at later times, is well-represented by this self-similar infall solution, but the departure from self-similarity, especially as related to the late tapering-off of the mass accretion is not.

\subsubsection{CDM haloes from non-self-similar spherical infall}

If the infall rate was given, instead, by the spherically-averaged, Lagrangian MAH of individual haloes in CDM N-body simulations, however, then the resulting haloes shared most of the empirical CDM $\mathrm{N}$-body halo structural properties and their time-evolution. In particular, in Alvarez et al. (2003), we took as our initial condition, a spherical density perturbation whose radial dependence ensured that the rate at which infalling mass reached the virial radius of the emerging halo followed the empirical MAH derived by Wechsler et al. (2002) from N-body simulations of CDM. With this simple assumption, we found that the virialized haloes which result have density profiles that are well-fit by the NFW density profile (at all radii $r / R_{\mathrm{vir}} \geq 0.01$ ), and the radius of the accretion shock is safely outside $R_{200}$. These halo density profiles also evolve like CDM N- body haloes, with concentration parameter that grows with time just as it does for CDM N-body haloes. In addition, as shown in Shapiro et al. (2006), the resulting halo phase-space density profile, $\rho / \sigma_{V}^{3}$, best-fit by a power-law $\propto r^{-1.93}$, was found to be in remarkable agreement at all times with the universal profile reported for CDM $\mathrm{N}$-body haloes. We concluded, therefore, that the time-varying mass accretion rate, or equivalently, the initial perturbation profile shape around the density peak that led to the formation of the halo, is the dominant influence on the internal structure of CDM haloes, which can be well-understood from this simple spherical infall model involving smooth accretion and the fluid approximation derived by Ahn \& Shapiro (2005) from the CBE.

\subsubsection{SFDM vs. CDM haloes from non-self-similar spherical infall}

In Paper I, we showed that the fluid approximation used above to model CDM halo dynamics also models SFDM dynamics in the freefield limit of FDM, as long as we do not need to resolve substructure on scales as small as the de Broglie wavelength but still wish to account faithfully for the influence of quantum pressure on larger scales. This, then, is a useful tool for the study of halo formation in the FDM regime, as long as we do not need to resolve the solitonic core in the central region. The results described above, which explain how the NFW profile and its related universal properties in the CDM model can arise as a simple consequence of spherical infall of smoothlyaccreted matter, also explain why 3D simulations of FDM haloes report NFW-like density profiles outside their solitonic cores.

It remains for us to determine here, what happens to this result when a repulsive SI is added to SFDM. In Paper I, we generalized the fluid approximation of Ahn \& Shapiro (2005) to include the effects of SI pressure in the TF regime, which corresponds to the regime in which the de Broglie wavelength can be assumed to be arbitrarily small, just right for application of the fluid approximation derived from the CBE. Our first approach to putting halo formation in the SFDM-TF model in a cosmological context will be to adopt the spherical infall model described above for the CDM model and solve the 1D, fluid conservation equations for SFDM-TF of Paper I with cosmological initial conditions to determine what the dynamical effect of adding SI to the scalar field is.

We consider a spherically-symmetric, adiabatic density perturbation as our initial condition. We take our initial time to be when the scale factor $a_{i}=a_{\mathrm{eq}}$, and further assume that the universe remains matter-dominated, thereafter, at the critical density, corresponding to an Einstein-de Sitter (EdS) cosmology. The initial linear density perturbation profile can be described in terms of the mass perturbation $\Delta_{\mathrm{L}}\left(M, a_{i}\right)$, where $M$ is the mass inside a sphere of initial (perturbed) radius $r\left(M, a_{i}\right)$, related to the mass $M_{b}\left(M, a_{i}\right)$ that would have been inside a sphere of that radius in the unperturbed background universe, according to

$\Delta_{\mathrm{L}}\left(M, a_{i}\right) \equiv \frac{M-M_{b}\left(M, a_{i}\right)}{M_{b}\left(M, a_{i}\right)}$

where $M_{b}\left(M, a_{i}\right)=(4 \pi / 3) r^{3}\left(M, a_{i}\right) \rho_{b}\left(a_{i}\right)$, and $\rho_{b}\left(a_{i}\right)$ is the background matter density at the initial redshift, given by $\rho_{b}\left(a_{i}\right)=$ $\rho_{\text {crit }}\left(a_{i}\right) \equiv 3 H^{2}\left(a_{i}\right) / 8 \pi G$, where the Hubble parameter evolves according to $H^{2}(a)=H_{0}^{2} a^{-3}$. We adopt the value $H_{0}=67 \mathrm{~km} / \mathrm{s} / \mathrm{Mpc}$ for the present-day Hubble parameter. The initial radius of the Lagrangian mass shell which encloses that mass $M$ is then given by

$r\left(M, a_{i}\right)=\left(\frac{M}{\frac{4 \pi}{3}\left[1+\Delta_{\mathrm{L}}\left(M, a_{i}\right)\right] \rho_{b}\left(a_{i}\right)}\right)^{1 / 3}$ 
while its initial velocity corresponds to the perturbed Hubble flow for the growing mode of the perturbation with that linear overdensity, given by (see, e.g., Mo, van den Bosch \& White 2010, §5.1.1)

$v\left(M, a_{i}\right)=H\left(a_{i}\right) r\left(M, a_{i}\right)\left(1-\Delta_{\mathrm{L}}\left(M, a_{i}\right) / 3\right)$

The quantity $\Delta_{\mathrm{L}}\left(M, a_{i}\right)$ is also the average fractional overdensity inside the sphere which encloses the mass $M$ at $a_{i}$. This average overdensity is chosen to be a function of mass $M$ such that, for an initial linear perturbation with this profile in a CDM universe, the ensuing growth would lead to infall and nonlinear collapse that reproduces the average MAH of the virialized haloes that form in CDM N-body simulations, as described above. The density profiles of these N-body simulation haloes are well-fit by NFW profiles ${ }^{8}$ (Navarro et al. 1997):

$$
\begin{aligned}
& \rho=\frac{\rho_{\mathrm{crit}} A_{\mathrm{NFW}}}{\left(c_{\mathrm{NFW}} r / R_{200}\right)\left(1+c_{\mathrm{NFW}} r / R_{200}\right)^{2}} \\
& A_{\mathrm{NFW}}=\frac{200}{3} \frac{c_{\mathrm{NFW}}^{3}}{\ln \left(1+c_{\mathrm{NFW}}\right)-c_{\mathrm{NFW}} /\left(1+c_{\mathrm{NFW}}\right)}
\end{aligned}
$$

where $R_{200}$ is the radius within which the average density is $200 \rho_{\text {crit }}$, and $c_{\mathrm{NFW}}$ is the concentration parameter, defined by $c_{\mathrm{NFW}} \equiv R_{200} / R_{S}$, where $R_{S}$ is the "scale radius" at which the logarithmic slope of the density profile equals -2 . Wechsler et al. (2002) found that the MAH of CDM haloes in N-body simulations followed a simple rule - the logarithmic slope of the mass accretion is inversely proportional to the scale factor:

$$
\begin{aligned}
\frac{d \ln M_{200}}{d \ln a} & \propto \frac{1}{a} \\
\Rightarrow M_{200}(a) & =M_{\infty} \exp \left(-s a_{f} / a\right)
\end{aligned}
$$

where $M_{200}$ is the mass within $R_{200}, M_{\infty}$ is the asymptotic halo mass as $a \rightarrow \infty$, and $a_{f}$ is the "formation scale factor" at which the logarithmic slope equals $s$. By convention, $s=2$. In Alvarez et al. (2003) and Shapiro et al. (2004), we found an initial perturbation profile for an EdS universe that is consistent with this MAH, given by

$$
\Delta_{\mathrm{L}}\left(M, a_{i}\right)=\Delta_{\mathrm{L}, i} \ln \left(M_{\infty} / M\right)
$$

where $\Delta_{\mathrm{L}, i} \equiv \Delta_{\mathrm{L}}\left(M_{200}\left(s a_{f}\right), a_{i}\right)$ is the initial average linear overdensity within the shell whose ballistic motion brings it to a radius which corresponds to $R_{200}$ at $a=s a_{f}$. Since $a_{f}$ corresponds roughly to the "formation time" of the halo, we can linearly extrapolate this initial overdensity to $a_{f}$, when the (linearly-extrapolated) average overdensity should be roughly unity:

$\Delta_{\mathrm{L}, f}=\Delta_{\mathrm{L}, i} \frac{a_{f}}{a_{i}} \approx 1 \Longrightarrow \Delta_{\mathrm{L}, i} \approx \frac{a_{i}}{a_{f}}$

The exact value we use in our code is $\Delta_{\mathrm{L}, i}=0.8 a_{i} / a_{f}$, which we obtained by calculating the linearly-extrapolated overdensity of a spherical top-hat in an EdS universe that has collapsed to a nonlinear overdensity of 200, at $a=s a_{f}$. We show our calculation in Appendix A.

As described above in $\$ 3.2 .3$, the result of adopting this linear perturbation profile and simulating its growth and collapse in a CDM universe, using the fluid approximation of Ahn \& Shapiro (2005), is to produce a virialized region inside the accretion shock whose structure and evolution closely resembles that reported for CDM Nbody haloes. For example, not only are the halo profiles that result from these initial conditions well-fit by an NFW density profile at

\footnotetext{
8 Note that in Paper I, we denoted $A_{\mathrm{NFW}}$ as $\delta_{\mathrm{NFW}}$.
}

all times following the formation of that accretion shock, but the concentration parameter of that profile increases over time as the halo grows, according to

$c_{\mathrm{NFW}}=4.25 a / a_{f} \quad$ for $a \gtrsim a_{f}$

(Shapiro et al. 2004; Alvarez et al. 2003). This is close to the relationship reported by Wechsler et al. (2002) in their analysis of 3D N-body simulations: $c_{\mathrm{NFW}}=4.1 a / a_{f}$.

In practice, if we want to form an individual halo with a given mass and concentration parameter at a given scale factor, we combine equations (36) and (39) and solve them for the free parameters of this NFW-producing initial condition, $\left(M_{\infty}, a_{f}\right)$, that will yield the desired halo. For example, consider a $10^{9} \mathrm{M}_{\odot}$ halo with a concentration parameter of 18 at the present (the median value for this mass as per the mass-concentration relation found by Klypin et al. 2016). Equation (39) then gives $a_{f} \simeq 0.24$ for this halo, in which case equation (36) gives $M_{\infty} \simeq 1.6 \times 10^{9} \mathrm{M}_{\odot}$. The results of a simulation run using these values in the initial condition will be discussed below, in $\$ 3.3 .1$.

\subsubsection{Numerical Method}

All simulations of cosmological halo formation from spherical infall in this paper will use the same 1D, Lagrangian hydrodynamics method and set-up as described in Paper I. We adopt the same non-self-similar initial conditions described above in \$3.2.4 and Appendix A, for both CDM and SFDM-TF, starting from $a=a_{\mathrm{eq}}$. This is designed to allow us to compare the dynamical consequences of SFDM-TF and CDM directly, for evolution from the same initial perturbation. Unless otherwise noted, each simulation uses 2000 Lagrangian shells, logarithmically-spaced in mass.

\subsection{Simulation results for cosmological halo formation from spherical infall and collapse}

\subsubsection{CDM halo formation from NFW-producing infall rate}

In Fig. 1, we show results of our 1D numerical hydrodynamics simulation of the formation of a CDM halo, based upon the fluid approximation of Ahn \& Shapiro (2005) for CDM described above in $\$ 2.4$ and Paper I. We adopted the non-self-similar cosmological perturbation described in \$3.2.4 and Appendix A as our initial condition, starting at $a=a_{\mathrm{eq}}$. Our purpose here is to illustrate the fact that this fluid approximation for CDM (without the SI pressure term which must be added to represent SFDM-TF) faithfully reproduces the universal halo properties and their evolution over time of the NFW-profile haloes reported for CDM N-body simulations when the initial perturbation is tuned to produce a smooth accretion rate that matches the MAH from Wechsler et al. (2002). The results in Fig. 1 confirm what we previously reported in Shapiro et al. (2004), Alvarez et al. (2003), and Shapiro et al. (2006), as they should, since we are following the same procedure. Our purpose in the next section will be to show what difference is made if these same CDM-like initial conditions are used to simulate the formation of an SFDM-TF halo, instead, when the important dynamical effects of both quantum pressure and SI-pressure are properly taken into account.

\subsubsection{SFDM-TF halo formation from NFW-producing infall rate: (SFDM-TF dynamics $)+(C D M$-like initial conditions $)$}

In Figs. 2 and 3, we show illustrative results of our 1D numerical hydrodynamics simulations of SFDM-TF halo formation from 


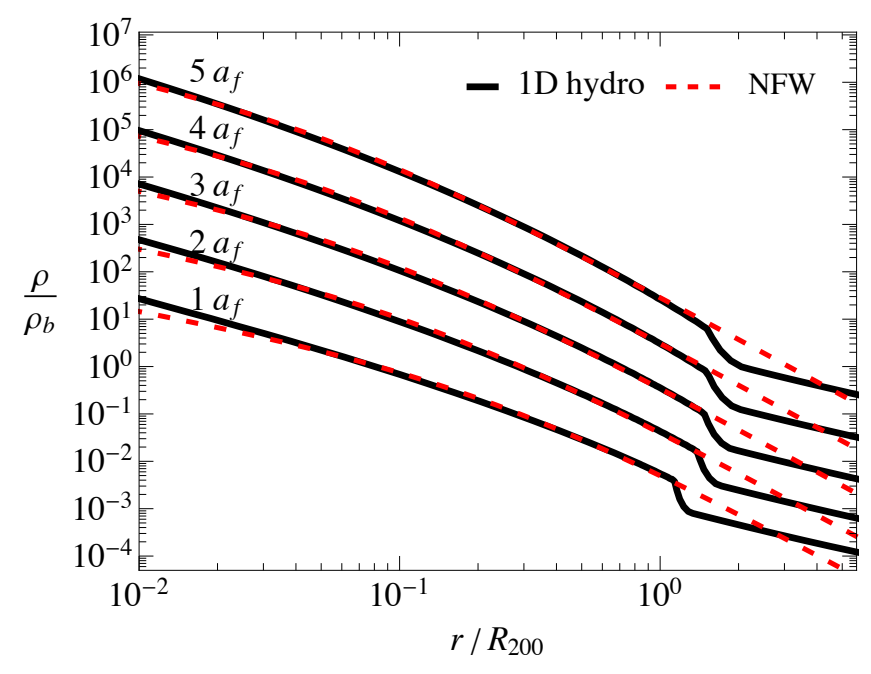

Figure 1. The fluid approximation results for halo formation from spherical infall and collapse in CDM vs. the NFW fit to $\mathrm{N}$-body simulations. Resulting density profile from our 1D hydrodynamics code without repulsive self-interaction $(g=0)$ starting from NFW-producing initial conditions. The red dashed line shows the corresponding NFW profile with the same value of $R_{200}$ as the numerical profile, but with $c_{\mathrm{NFW}}$ chosen to fit the numerical profile in the domain $10^{-2}<r / R_{200}<1$. The profiles are plotted at 5 scale factors, $a / a_{f}=\{1,2,3,4,5\}$, for which the best-fit NFW concentration parameters are $c_{\mathrm{NFW}}=\{4.6,8.5,12.7,17.0,21.2\}$, respectively. The curves for $a / a_{f}=1,2,3$, and 4 have been shifted downward by $4,3,2$, and 1 order(s) of magnitude, respectively, for visual clarity. The radius $R_{200}$ and background density $\rho_{b}$, used to normalize the radius on the x-axis and density on the y-axis, are evaluated at each time-slice, separately. As expected, these results reproduce those presented previously in Alvarez et al. (2003), Shapiro et al. (2004), and Shapiro et al. (2006).

the same initial conditions as used in the previous section for the CDM halo formation shown in Fig. 1. In this case, however, our fluid equations are now the modified ones for SFDM-TF, which include the dynamical effects of both SI pressure $P_{\mathrm{SI}}$ and quantum pressure, where the latter is accounted for by the ideal gas pressure $P_{\sigma}$ (i.e. CDM-like "velocity-dispersion" pressure), which represents the dynamical effect on large scales that results from inhomogeneity on the tiny, unresolved scale of the de Broglie wavelength. Two cases are shown in Fig. 2, involving different SI strengths, parameterized by their adopted $R_{\mathrm{TF}}$-values, with initial perturbations chosen to make haloes of different mass at $a=a_{f}$ in each case. Profiles of hydrodynamical variables vs. proper radius are plotted at selected times, including mass density, bulk velocity, ideal gas pressure (i.e. velocity-dispersion "pressure"), and "temperature" (i.e. velocity-dispersion), for two example SFDMTF haloes, formed from the same NFW-producing initial conditions at $a=a_{\mathrm{eq}}$ as for the CDM simulations in Fig. 1, with $\left\{M_{\infty}, a_{f}\left(z_{f}\right), R_{\mathrm{TF}}\right\}=\left\{5.7 \times 10^{14} \mathrm{M}_{\odot}, 0.87(0.15), 1 \mathrm{kpc}\right\}$ (left) and $\left\{1.6 \times 10^{9} \mathrm{M}_{\odot}, 0.24\right.$ (3.2), $\left.10 \mathrm{pc}\right\}$ (right). For comparison, we also plot the end results for CDM, from the same initial conditions.

The evolution is similar in both cases, with some elements common to the non-cosmological infall and collapse we simulated in Paper I, from static, Jeans-unstable initial conditions, while others are uniquely cosmological, as follows:

(1) Growth of the initial perturbation slows its expansion relative to the unperturbed Hubble flow far from the center, so the per- turbed density in units of the time-dependent mean background density increases with time, even as the latter drops.

(2) Close to the center, a sound wave driven by the SI pressure gradient quickly propagates outward until it reaches the physical radius $R_{\mathrm{TF}}$, one sound-crossing-time after the initial time at $a_{i}$, travelling at the SI sound speed given by

$c_{s, \mathrm{SI}}^{2}=\frac{\partial P_{\mathrm{SI}}}{\partial \rho}=\frac{g \rho}{m^{2}}$

evaluated at a density roughly equal to the initial background density. This sound wave smooths out the density as it goes, leaving it nearly uniform within this radius, and remaining uniform as it expands, i.e. it expands homologously. We will say more about this sound-wave-smoothed central region below.

(3) Thereafter, all mass shells continue expanding until - at later times for shells that started at larger radii - they reach maximum expansion, halt and collapse inward, supersonically.

(4) As the density of these infalling shells rises, adiabatic compression increases their ideal gas pressure more slowly (since $P_{\sigma} \propto \rho^{5 / 3}$ ) than it does their SI pressure (for which $P_{\mathrm{SI}} \propto \rho^{2}$ ). The latter eventually becomes large enough in the center to halt the infall of the innermost shells and provide their hydrostatic support within a radius $\simeq R_{\mathrm{TF}}$, with a flattened profile like that of an $(n=1)$-polytrope. This was also seen in the non-cosmological results of Paper I.

(5) At this point, by $a / a_{f} \gtrsim 0.4$, the incoming mass shell just outside this polytropic core - just about to slam into it - is halted by the formation of a strong accretion shock that converts its kinetic energy of infall into "thermal energy" (i.e. energy due to dispersion). This boosts its ideal gas pressure to be comparable to the SI pressure within the core and extends the hydrostatic inner profile of the latter to larger radii, bounded by the shock, supported outside the core by post-shock $P_{\sigma}$, instead of $P_{\mathrm{SI}}$. This, too, is similar to the non-cosmological case in Paper I.

(6) By $a / a_{f}=1$, the accretion shock has moved outward in mass, just beyond the radius $R_{200}$, to encompass a virialized halo, within which the "temperature" profile associated with velocity dispersion is approximately isothermal, except inside the polytrope, where it is cold, because it remained interior to the mass shell at which the shock formed. However, matter interior to that shock is everywhere hydrostatic, supported inside the polytropic core by SI pressure and outside it by post-shock ideal gas pressure.

(7) The post-shock density profile, thereafter, has three distinct regions in radius:

(a) a flattened, polytropic core inside $R_{\mathrm{TF}}$,

(b) an intermediate zone with power-law density profile, $\rho \propto$ $r^{-12 / 7}$, and

(c) an outer envelope which follows the same NFW-profile as for CDM from the same initial conditions, inside the advancing accretion shock, which remains just outside of the time-varying $R_{200}$.

Regions 7(a) and (b) were also present in the non-cosmological results in Paper I, while 7(c) is unique to the cosmological case.

(8) Outside the shock, the density follows the pre-shock, ballistic infall profile of a cold, pressure-free gas, just like the CDM case.

As described in $\$ 3.1$, Paper I found a generic core-envelope structure for the virialized objects formed by the nonlinear evolution of the Jeans instability in SFDM-TF from non-cosmological (i.e. static) initial conditions. As in the cosmological case described above, an accretion shock bounded the virialized region. However, unlike the cos- 

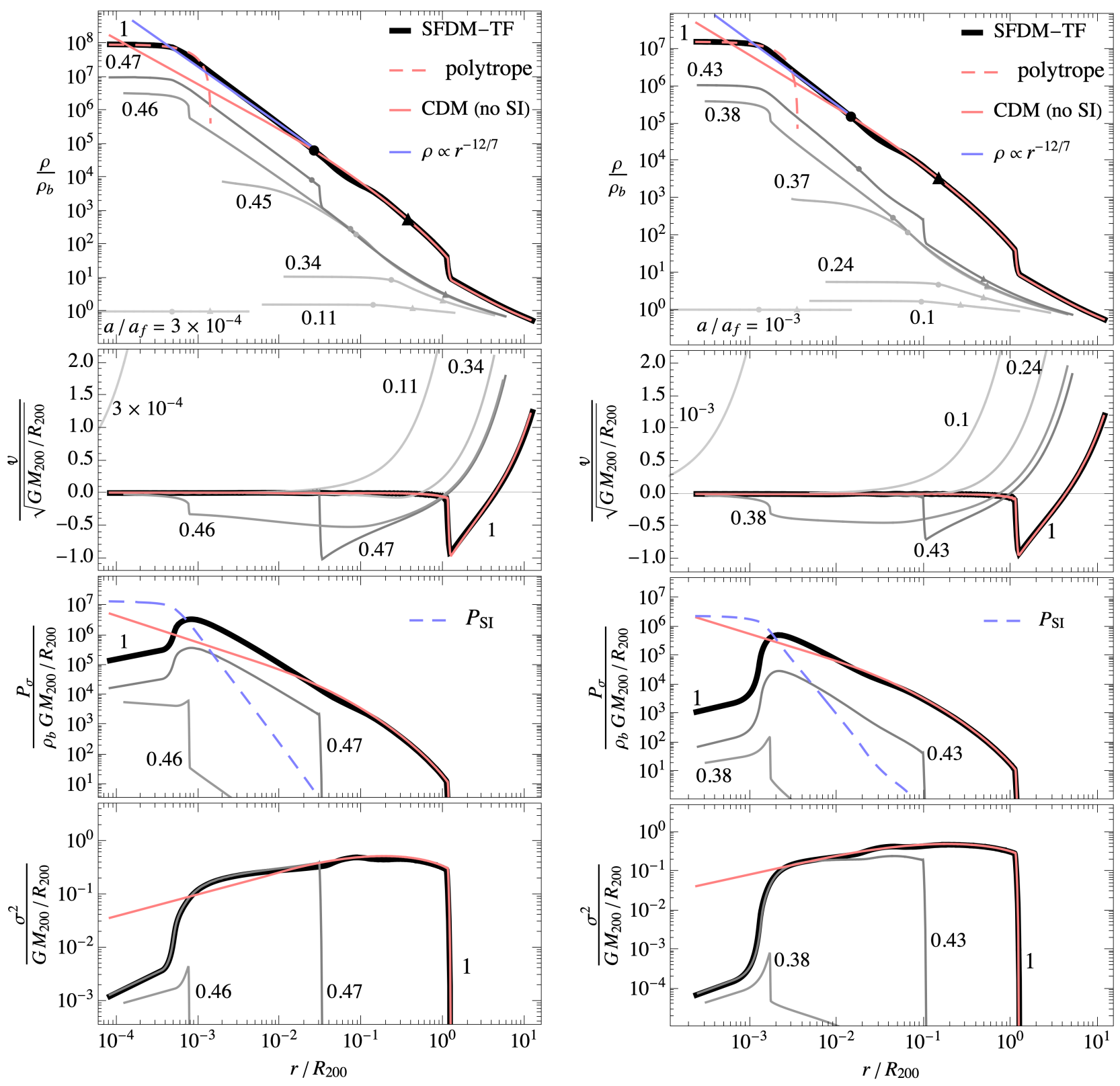

Figure 2. The fluid approximation simulation results for SFDM-TF halo formation from spherical infall and collapse of a cosmological perturbation. Each panel shows profiles of hydrodynamical variables at select times: from top to bottom, (non-dimensionalized) mass density, bulk velocity, ideal gas pressure (i.e. velocity-dispersion "pressure"), and "temperature" (i.e. velocity dispersion) vs. radius, for two example SFDM-TF haloes, from the same NFWproducing initial conditions at $a=a_{\mathrm{eq}}$ as for the CDM simulations in Fig. 1, with $\left\{M_{\infty}, a_{f}\left(z_{f}\right), R_{\mathrm{TF}}\right\}=\left\{5.7 \times 10^{14} \mathrm{M}_{\odot}, 0.87(0.15), 1 \mathrm{kpc}\right\}$ (left) and $\left\{1.6 \times 10^{9} \mathrm{M}_{\odot}, 0.24\right.$ (3.2), $\left.10 \mathrm{pc}\right\}$ (right). The thick black lines show the profiles at the "formation" scale factor $a=a_{f}$. The thin grey lines show the profiles at earlier times in the simulation, labeled by $a / a_{f}$. Here, $\rho_{b}$ is the time-dependent background density, while $\left\{M_{200}, R_{200}\right\} \simeq\left\{7 \times 10^{13} \mathrm{M}_{\odot}, 740 \mathrm{kpc}\right\}$ (left) and $\left\{2 \times 10^{8} \mathrm{M}_{\odot}, 3 \mathrm{kpc}\right\}$ (right) are the mass and radius of the halo at $a=a_{f}$. The discontinuity near $R_{200}$ is the accretion shock, which sets the virial radius of the halo. The thin red lines are profiles for a corresponding CDM run using the same initial conditions. The SFDM-TF density profile closely tracks the NFW-like $\mathrm{CDM}$ profile at large radii, but more closely resembles a power-law, $\rho \propto r^{-12 / 7}$, at smaller radii (blue lines), until, at still smaller radii, $r \lesssim R_{\mathrm{TF}}$, flattens to the shape of an SI-supported polytropic core (red long-dashed lines). For SFDM-TF at early times, the centrally-peaked initial density profile was flattened, out to $\sim R_{\mathrm{TF}}$, by an outward-propagating, SI-pressure-driven sound wave. Circles mark the shell that was located at $r=R_{\mathrm{TF}}$ at the time the sound wave reached that radius, while triangles mark the shell that was located at $r=R_{\mathrm{TF}}$ at the initial time $a_{i}$-these are indicators of where the profile deviates from CDM. The long-dashed blue line in the $P_{\sigma}$ panel shows the profile for $P_{\mathrm{SI}}=g \rho^{2} / 2 m^{2}$ at $a=a_{f}$ with the same normalization.

mological case above, the entire envelope between the polytropic core and the shock had the same power-law density profile $\rho \propto r^{-12 / 7}$ as reported above for the intermediate zone 7(b), and there was no NFWlike zone 7(c). This reflected the fact that, in this non-cosmological case, the Jeans-instability problem involved a nearly uniform initial density, which gravitational instability turned into an infall density profile (outside the accretion shock) with that power-law. The condition of hydrostatic equilibrium inside the post-shock region then combined with the constant (shock-velocity-independent) factor of density jump at the shock to make the profile of post-shock den- 

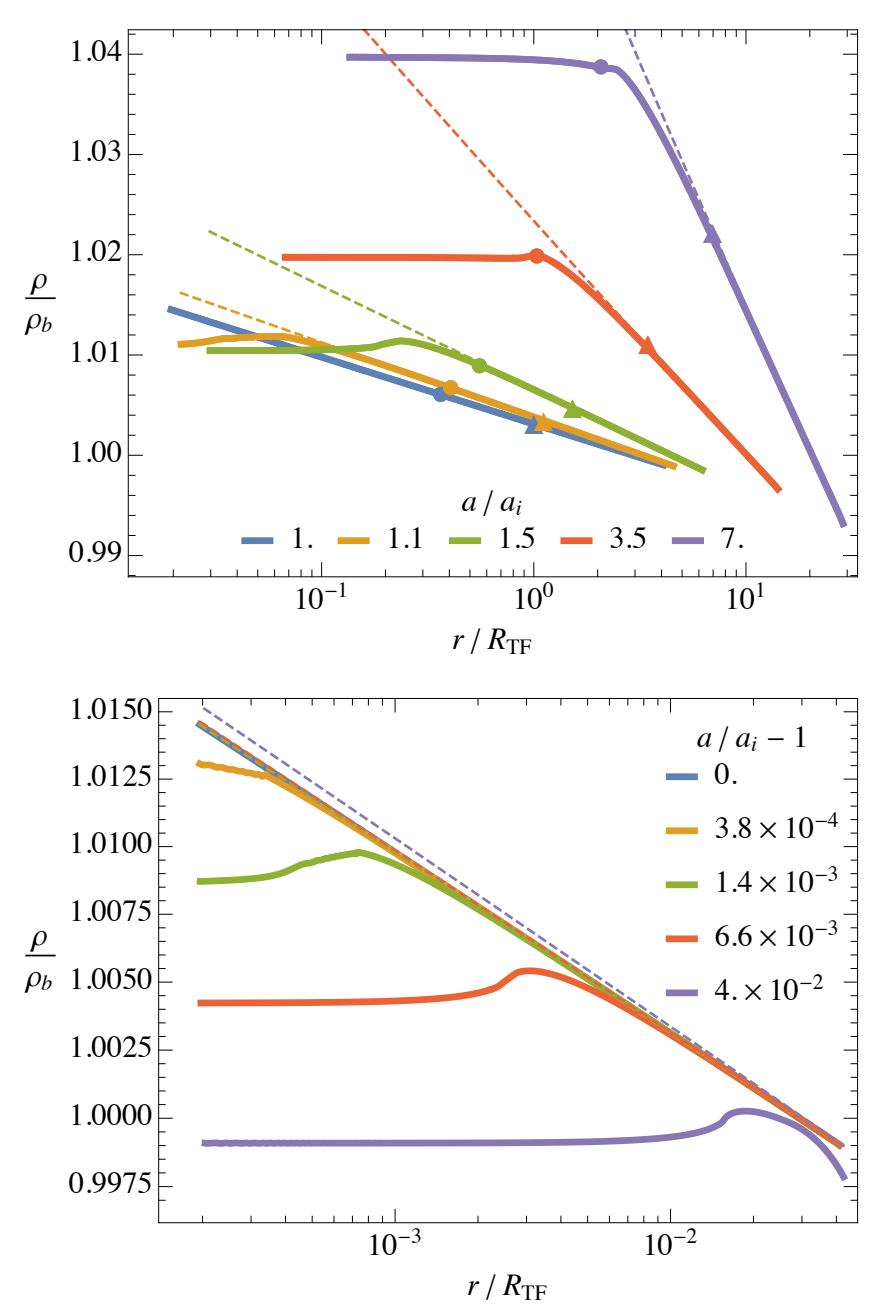

Figure 3. The beginning of spherical collapse from NFW-producing initial conditions: early Jeans-filtering by SI-pressure. Simulation results at several early time-slices, soon after the initial scale factor $a_{i}$, for two cases with the same initial perturbation, with $M_{\infty}=1.6 \times 10^{9} \mathrm{M}_{\odot}$ and $a_{f}=0.24$, but with different SI strengths for SFDM-TF: (a) (top panel) $R_{\mathrm{TF}}=10 \mathrm{pc}$, and (b) (bottom panel) $R_{\mathrm{TF}}=1 \mathrm{kpc}$. Corresponding CDM runs are shown for comparison (dashed lines). The density profiles are normalized by the time-dependent background density. The figures illustrate the initial SI sound wave that propagates outward and flattens the density profile out to $R_{\mathrm{TF}}$. In the case of $R_{\mathrm{TF}}=1 \mathrm{kpc}$, this sound wave passes over the entire profile (which encompasses the entire computational domain, extending to $M_{\infty}$ ), and the perturbation is prevented from growing (i.e. it is Jeans-filtered) within the age of the universe. Circles mark the shell that was located at $r=R_{\mathrm{TF}}$ at the time the sound wave reached that radius, while triangles mark the shell that was located at $r=R_{\mathrm{TF}}$ at the initial time $a_{i}$.

sity the same as that of the pre-shock infall. Indeed, for this reason, our simulation from the same non-cosmological initial conditions but with CDM dynamics, instead, produced the exact same powerlaw profile inside the shock, except with no SI-flattened polytropic core in the center. In fact, the mass inside the core in the SFDM-TF case, with polytropic radius $R_{\mathrm{TF}}$, was the same as that inside that radius in the CDM-like power-law profile. So, apparently, outside of $R_{\mathrm{TF}}$, where SFDM-TF and CDM dynamics was the same, it was the non-cosmological initial conditions of the SFDM-TF and CDM simulations that resulted in making their infall profiles $\rho \propto r^{-12 / 7}$, instead of the cosmological infall profile derived from the halo mass accretion rate in CDM N-body simulations, which makes the postshock profile NFW-like.

From that description, we might reasonably have expected halo formation from cosmological initial conditions to be similar, with the same polytropic core, surrounded again by a CDM-like envelope. In particular, we might have expected this CDM-like envelope to be $N F W$-like, as it is in our CDM simulation (without SI) for this same cosmological case. Then why did we find, as reported in item (7), that while this is true for regions (a) and (c), there is an intermediate zone (b) in which the density profile follows the same power-law profile $\left(\rho \propto r^{-12 / 7}\right)$ as in the non-cosmological simulations of Paper I, instead? The answer to this question encapsulates the fundamental distinction between static and cosmological Jeans instability in SFDM-TF and is at the heart of our cosmological story-line here in Paper II.

What distinguishes a cosmological from a non-cosmological density perturbation is their different initial velocity perturbations. Within the Newtonian approximation, our spherically-symmetric Jeans instability problem is transformed from a non-cosmological one into a cosmological one entirely by the presence of perturbed Hubble expansion in the latter that replaces the static initial condition of the former. This small change has profound consequences, however, because the SI Jeans length is fixed in physical coordinates by the constant value of $R_{\mathrm{TF}}$. For CDM in an Einstein-de Sitter universe, a spherical perturbation that is overdense will expand more slowly than unperturbed Hubble expansion, until it eventually reaches a maximum expansion radius and recollapses. This will be true in SFDM-TF, as well, if it is overdense across a region much larger than $R_{\mathrm{TF}}$. But in that case, matter close to the center of the perturbation, which is initially expanding, will be affected by the presence of SI pressure, too, which responds to density inhomogeneity by driving a sound wave outward from the center, as far as $R_{\mathrm{TF}}$, which smooths out the density perturbation as it goes. This is what we found in item (2) above. The effect of this early SI-smoothing imprints the evolution to come for the mass shells inside the sphere smoothed-out by the sound wave, even though they continue to expand homologously, thereafter, which carries them far outside the physical radius originally overtaken by the sound wave.

In Fig. 3, we illustrate this early SI-sound-wave smoothing by two examples from the same NFW-producing CDM-like initial perturbation, with $M_{\infty}=1.6 \times 10^{9} \mathrm{M}_{\odot}$ and $a_{f}=0.24$, but with different SI strengths, by plotting density profiles for a sequence of early time-slices of our SFDM-TF simulation, compared with their CDM counterparts. Let us focus first on the top panel, Fig. 3(a), the case for $R_{\mathrm{TF}}=10 \mathrm{pc}$. The central density profile is quickly made nearly uniform out to $R_{\mathrm{TF}}$, by a sound wave from the origin that reaches $R_{\mathrm{TF}}$ long before that shell reaches maximum expansion. Thereafter, this inner sphere evolves just like a top-hat perturbation. As such, it continues to expand until it reaches maximum expansion, turns around, and recollapses. At maximum expansion, the spherical region is momentarily at rest, so it is at that moment essentially the same as the initial perturbation of the non-cosmological problem in Paper I! Its evolution, thereafter, must, therefore, be similar to what we found there. It should yield the same core-envelope structure inside the advancing accretion shock as in Paper I, with polytropic core of proper radius $R_{\mathrm{TF}}$ and the same power-law density profile, $\rho \propto r^{-12 / 7}$, outside the core, but only out through the mass that was initially smoothed by the SI-pressure-driven sound wave. As we can see in Fig. 3, the sound wave smooths out the density as it travels to $\sim R_{\mathrm{TF}}$. Each time-slice in Fig. 3(a) is marked (by a filled circle) to identify the particular mass shell that was located at $R_{\mathrm{TF}}$ when 
the sound wave reached that radius. The mass within that radius was basically the SI-Jeans mass of the background universe at that time, since the density perturbation was only linear then. The time-varying radius so marked, which contains this fixed mass as it evolves, provides a rough estimate (i.e. lower-limit) for the outer radius of the intermediate zone 7(b) within which we expect the final density profile to follow the power-law $\rho \propto r^{-12 / 7}$, once this mass is inside the shock. We also mark this radius on the density profiles in the top panels of Fig. 2, for all the time-slices, to confirm this.

The transition from power-law to NFW-like post-shock density profiles inside the shock extends beyond the filled circles, encompassing a somewhat larger mass. (The filled circles in Fig. 2 enclose masses of $5 \times 10^{11} \mathrm{M}_{\odot}$ (left) and $5 \times 10^{5} \mathrm{M}_{\odot}$ (right), for $R_{\mathrm{TF}}=1$ $\mathrm{kpc}$ and $10 \mathrm{pc}$, respectively, as compared with their Jeans masses at the initial time of $2 \times 10^{13} \mathrm{M}_{\odot}$ and $2 \times 10^{7} \mathrm{M}_{\odot}$.) We can understand why and bound this transition from above by a second estimate, as follows, which we mark with filled triangles on the density profiles in both Figs. 2 and 3(a). As we shall see, in §4, the cosmological SI Jeans mass at any scale factor is the mass inside a sphere of fixed physical radius $R_{\mathrm{TF}}$, containing the mean density at that time, a mass which drops with scale factor as $a^{-3}$ (see equation 58). As a result, even the small, finite time for the sound wave to reach $R_{\mathrm{TF}}$ is enough time for the Jeans mass to drop significantly from its value at the initial scale factor $a_{i}$. Therefore, the effects of SI Jeans-filtering extend to encompass a mass which is between this (larger) initial Jeans mass (marked by filled triangles) and the (smaller) Jeans mass when the sound wave reached $R_{\mathrm{TF}}$ (marked by filled circles).

Now we are ready to focus on the second example of SI-smoothing, plotted in the bottom panel Fig. 3(b). This case differs from that in the top panel Fig. 3(a) only by having a much stronger SI, corresponding to $R_{\mathrm{TF}}=1 \mathrm{kpc}$, instead of $10 \mathrm{pc}$. As with the latter case, the initial SI sound wave propagates outward and flattens the density profile out to $R_{\mathrm{TF}}$. In the case of $R_{\mathrm{TF}}=1 \mathrm{kpc}$, however, this sound wave passes over the entire computational domain, which extends to $M_{\infty}$ (i.e. the radial position of the outermost shell containing $M_{\infty}$ is $\ll R_{\mathrm{TF}}$ ), and the perturbation is, thereby, prevented from growing to form the collapsed virialized object it would have formed in the CDM case.

This reveals the essential factor that distinguishes the outcomes of non-cosmological vs. cosmological initial conditions for the formation of an SFDM-TF halo with a given mass at a given epoch. The former yields a virialized halo in the post-shock region, with a polytropic core of radius $R_{\mathrm{TF}}$ surrounded by a CDM-like density profile that extends to the accretion shock radius at that epoch. The mass encompassed by that shock grows with time just by adding new mass shells to the outside, at the gravitational free-fall times defined by the mean mass densities initially interior to each mass shell. The mass in the core is fixed by extrapolating the CDM-like profile inward from $R_{\mathrm{TF}}$ and integrating out to that radius. SI-smoothing in this case is confined to the region within a fixed physical radius $R_{\mathrm{TF}}$, and to the mass which ends up inside this radius at the final epoch, while the dynamics outside this radius - and the final halo structure there are CDM-like. For the cosmological case, however, the mass that is SI-smoothed is the much larger amount that was inside that same sphere of physical radius $R_{\mathrm{TF}}$ at a time close to the initial time, at the mean matter density of the universe at that time, before expansion carried it well beyond $R_{\mathrm{TF}}$, enroute to its turn-around radius. Since SI-smoothing quickly flattens the initial perturbation out to $R_{\mathrm{TF}}$, the turn-around and recollapse of any shell interior to the one which bounds the smoothed mass will be delayed relative to their timing without SI-smoothing. That bounding sphere and every shell inside it will expand, turn-around and recollapse like a uniform, top-hat perturbation with the same mean density. Since SI-smoothing out to
$R_{\text {TF }}$ must conserve mass, the average overdensity inside the smoothed sphere must be about the same as in the unsmoothed perturbation, so the bounding sphere and its contents turn-around and recollapse just like the bounding sphere in the case without SI-smoothing. As long as the latter is destined to collapse, that means SI-smoothing does not prevent the smoothed mass from collapsing, it only delays the collapse of interior shells to the time at which the bounding sphere, itself, would have, in the absence of SI-smoothing.

This assumes, of course, that the average overdensity inside the bounding sphere in the initial perturbation was positive, as it would be for the self-similar perturbations described above in $\$ 3.2 .2$. The CDM-like perturbation that leads to an NFW-like post-shock profile, however, is a non-self-similar one, in which the overdensity is "compensated" by an underdensity at large radii, so that the average overdensity inside the sphere which encompasses mass $M_{\infty}$ is zero. Spherical shells outside that mass will, therefore, remain unperturbed and cannot collapse. In that case, if the mass affected by SI-smoothing exceeds $M_{\infty}$, as it does in Fig. 3(b), then the bounding sphere described above is actually prevented from collapsing, altogether. For those cases that $d o$ collapse, however, our simulations above tell us that the virialized object that forms inside the accretion shock will have an inner portion that resembles that in Paper I from non-cosmological (static) initial conditions, encompassing the initial SI-smoothed mass, with the core-envelope structure reported there (polytropic core of radius $R_{\mathrm{TF}}$, surrounded by a power-law density profile, $\rho \propto r^{-12 / 7}$ ), but beyond the radius containing this initial SI-smoothed mass, it will transition to the NFW-like profile.

This description above assumes we started from CDM-like initial conditions. We made this assumption as the first step in understanding the fully nonlinear dynamics of SFDM-TF halo formation. That was a reasonable first step for a range of perturbation mass scales, in view of our discussion in Paper I of the fact that, at scales beyond $R_{\mathrm{TF}}$, the dynamics of SFDM-TF is CDM-like. As such, we were able to confirm that, beyond the inner mass smoothed by SI, SFDMTF halo profiles simulated with nonlinear dynamics will follow the same NFW-like profile expected for the CDM model. However, we also learned that this SI-smoothed inner mass can be as large as the mass inside the fixed radius $R_{\mathrm{TF}}$ at early times, when the universe was much denser, and that there is an intermediate zone of density profile outside the polytropic core of radius $R_{\mathrm{TF}}$ in the final object, which differs from the NFW profile. Such a feature might be a signature of SFDM-TF that distinguishes it observationally from CDM. However, it remains to be seen from 3D-cosmological SFDM-TF simulations, from initial conditions that are self-consistently based upon linear perturbation theory for SFDM-TF, if this intermediate zone will survive, or else relax to the NFW-like profile over time.

In addition, such $3 \mathrm{D}$ simulations are required to determine what MAH results for halo growth from SFDM initial conditions and how it differs from that for CDM. The results we present here by assuming the smoothed accretion rate derived from N-body simulations of the CDM model are likely to be robust on the smallest and largest scales with respect to replacing the initial conditions with the proper MAH of SFDM. The shells at large radii are likely to follow CDM-like trajectories even with correct initial conditions, while the shells at small radii are quickly smoothed by SI Jeans filtering and so should also follow the correct trajectories.

As we shall see below, from linear perturbation analysis, SFDM initial conditions differ from those of CDM by suppressing power on small scales, due to Jeans filtering. Halos can form, however, on all mass scales, even those below that filter scale, although with lower abundance for those smaller masses. Filtering may smooth the initial density perturbation responsible for building a halo by 
spherical infall, out to a larger radius than in the case with CDMlike initial conditions, resulting in a more nearly uniform collapse. In that case, we might expect the nonlinear collapse to produce a post-shock density profile outside the polytropic core, which follows the power-law $\rho \propto r^{-12 / 7}$, over a larger range of radii than in our results above.

In the meantime, we need linear perturbation theory to determine the statistical measures of structure formation, e.g. how many haloes of a given mass are likely to form by a given epoch, in order to constrain the model parameter $R_{\mathrm{TF}}$ against observations. We consider that in the remainder of this paper.

\section{LINEAR PERTURBATION THEORY AND THE TRANSFER FUNCTION FOR SFDM-TF}

\subsection{Qualitative overview}

The growth of density perturbations in a universe with complex SFDM in the presence of a repulsive SI differs from both that in the CDM model and that in the Fuzzy Dark Matter (FDM) limit of SFDM. In all models, the assumed, primordial initial condition involves small-amplitude, 3D, Gaussian-random density fluctuations with zero mean, characterized by a scale-invariant ${ }^{9}$ power spectrum

$P\left(k, t_{i}\right)=\left|\delta_{k}\left(t_{i}\right)\right|^{2} \propto k$

where the linear overdensity $\delta_{k}(t)$ of a Fourier mode of wavenumber $k$ at a cosmic time $t$ is related to its amplitude $\delta_{k}\left(t_{i}\right)$ at initial time $t_{i}$, according to

$\delta_{k}(t)=\mathcal{T}_{k}\left(t, t_{i}\right) \delta_{k}\left(t_{i}\right)$

in terms of the transfer function $\mathcal{T}_{k}\left(t, t_{i}\right)$. Differences in the structure formation histories of these models arise while they are still in their linear amplitude phase, as characterized by different transfer functions for each model. They have one very important thing in common, however. When fluctuation modes of different proper wavelength $\lambda$ (or, equivalently, comoving $k=2 \pi a / \lambda$ ) first re-enter the horizon (i.e. near the time $t=t_{\mathrm{H}}$ at which their proper wavelength equals the Hubble diameter, i.e. $\lambda(t)=2 c / H(t)$, in which case comoving $\left.k=k_{\mathrm{H}} \equiv \frac{\pi}{c} a_{\mathrm{H}} H\left(a_{\mathrm{H}}\right)\right)$, they do so with the same, wavelength-independent, fractional overdensity, $\delta_{k}\left(t_{\mathrm{H}}\right) \ll 1$, for all models. Although modes of different $k$ enter the horizon at different times $t_{\mathrm{H}}$ (later for smaller $k$ ), and it is even possible that some modes of the same $k$ in the different models enter the horizon at different times, the value of $\delta_{k}\left(t_{\mathrm{H}}\right)$ in all cases is the same for all values of $k$ and all values of $t_{\mathrm{H}}$.

The value of $t_{\mathrm{H}}$ for a given wavenumber depends only on the timedependence of the Hubble parameter, so $t_{\mathrm{H}}(k)$ is the same in all models only if $H(t)$ is the same at all times in different models. We expect this to be the case for CDM and SFDM models, in general, in our observed Universe, for modes that re-enter the horizon during the radiation-dominated (RD) and matter-dominated (MD) eras of interest to us here, since all models are required by observational constraints to share a common expansion history from the time the wavenumbers associated with galaxy formation began to enter the horizon during the RD era through the present. This condition is not met for all of the models at all times. For modes that enter before

\footnotetext{
9 More generally, $P\left(k, t_{i}\right) \propto k^{n_{s}}$ where $n_{s}$ is the primordial spectral index of scalar perturbations, and the value we adopt here is $n_{s}=1$, the HarrisonPeebles-Zeldovich spectrum, which is consistent with current observational constraints.
}

\begin{tabular}{cc}
\hline \hline parameter & value \\
\hline$h$ & 0.67 \\
$\Omega_{\mathrm{dm}} h^{2}$ & 0.12 \\
$\Omega_{m} h^{2}$ & 0.14 \\
$\Omega_{\Lambda} h^{2}$ & 0.31 \\
$\Omega_{\mathrm{rad}} h^{2}$ & $4 \times 10^{-5}$ \\
\hline
\end{tabular}

Table 1. Cosmological parameters from Planck Collaboration et al. (2020), which we adopt for our linear perturbation analysis in $\S 4$.

the RD era - e.g. for complex SFDM, with or without SI, there is an early stiff phase $(w=1)$, prior to the RD era, in which the scalar field dominates. Hence, even though the Hubble parameter during the RD and MD eras, for example, is basically the same at a given scale factor for all models, it differs during the SFDM stiff phase at earlier times, in that case, when much smaller scales entered the horizon. In fact, even during the RD epoch, but well before the epoch of matter-radiation equality, $a_{\mathrm{eq}}$ (for both complex and real field cases), the Hubble parameter can be slightly enhanced relative to its value in CDM and FDM, by a small boost to the total energy density contributed by SFDM in the presence of SI, when its EOS is radiation-like $(\langle w\rangle=1 / 3)$, between its stiff- and matter-like phases of evolution, but this small enhancement is gone well before $a_{\mathrm{eq}} \cdot{ }^{10}$

What happens to that amplitude next, after a mode is already inside the horizon, depends upon the microscopic nature of the dark matter and other components that contribute to the total mass-energy of the Universe. It also depends on whether the background Universe is RD or MD during the phase where the galaxy- and galaxy-cluster-scale fluctuations are still linear.

In the standard CDM model, dark matter is a nonrelativistic gas of cold, collisionless particles, so the ratio $w$ of its pressure to its energydensity is well-approximated by $w=0$. In that case, its fluctuation growth rate after horizon entry depends upon whether the Universe is in the $\mathrm{RD}$ or MD era. For the scales of interest for structure formation, spanning the range of galaxy- and cluster-mass objects, horizon entry is during the RD era. At that time, radiation dominates the gravitational potential fluctuations that drive perturbation growth in CDM, but fluctuations in the radiation component do not amplify during this phase, because a relativistic fluid with $w=1 / 3$ has a speed of sound close to $c / \sqrt{3}$, and density inhomogeneities are thus smoothed out by pressure disturbances that can travel a distance comparable to the Hubble radius within the age of the universe at that time. As a result, gravitational fluctuation growth in the CDM component is driven only by its own fluctuation as a subdominant contribution to the total energy-density. This yields only a relatively small amount of growth between horizon entry at scale factor $a_{\mathrm{H}}$, and matter-radiation equality at $a_{\mathrm{eq}}$, which is a logarithmic function of those scale factors.

We adopt cosmological parameters from Planck Collaboration et al. (2020) as given in Table 1. The scale factor of matter-radiation equality is given by

$a_{\mathrm{eq}} \simeq 2.9 \times 10^{-4}$

For a comoving wavelength $\lambda_{0}$ that enters the Hubble volume during

10 When we describe the EOS parameter $w$ of the scalar field during its rapid-oscillation phase, in which the value averaged over oscillation periods, $\langle w\rangle$, evolves from $\langle w\rangle=1 / 3$ to $\langle w\rangle=0$, we will, henceforth, generally just write $w$ to refer to this averaged quantity and omit the brackets. 
the RD epoch,

$a_{\mathrm{H}} \simeq 10^{-6}\left(\frac{\lambda_{0}}{1 \mathrm{Mpc}}\right)$

And the unperturbed mass of nonrelativistic dark matter contained in a sphere of proper radius $\lambda(t) / 2$, where $\lambda(t) / a(t)=\lambda_{0} / a_{0}$, is given at all times by

$M=\frac{4 \pi}{3} \rho_{\mathrm{dm}, 0}\left(\frac{\lambda_{0}}{2}\right)^{3} \simeq 1.45 \times 10^{11} \mathrm{M}_{\odot}\left(\Omega_{\mathrm{dm}} h^{2}\right)\left(\frac{\lambda_{0}}{1 \mathrm{Mpc}}\right)^{3}$

where the mean dark matter density at present is given by

$\rho_{\mathrm{dm}, 0} \equiv \Omega_{\mathrm{dm}} \frac{3 H_{0}^{2}}{8 \pi G} \simeq 2.8 \times 10^{11} \frac{\mathrm{M}_{\odot}}{\mathrm{Mpc}^{3}}\left(\Omega_{\mathrm{dm}} h^{2}\right)$

We can also write the scale factor when a given mass fills the Hubble volume, according to

$a_{\mathrm{H}} \simeq 1.9 \times 10^{-6}\left(\Omega_{\mathrm{dm}} h^{2}\right)^{-1 / 3}\left(\frac{M}{10^{12} \mathrm{M}_{\odot}}\right)^{1 / 3}$

The comoving wavelength that entered the horizon at $a_{\mathrm{eq}}$ is given by

$\lambda_{0, \mathrm{eq}} \simeq 290 \mathrm{Mpc}$

and the mass ${ }^{11}$ that filled the Hubble volume at $a_{\mathrm{eq}}$ is, therefore, given by

$M_{\mathrm{H}, \mathrm{eq}} \simeq 3.5 \times 10^{18} \mathrm{M}_{\odot}\left(\Omega_{\mathrm{dm}} h^{2}\right)$

which, for $\Omega_{\mathrm{dm}} h^{2}=0.12$, yields $M_{\mathrm{H}, \mathrm{eq}} \simeq 4 \times 10^{17} \mathrm{M}_{\odot}$.

For CDM, modes of mass scale $M$ that enter the horizon during RD experience only logarithmic growth between $a_{\mathrm{H}}$ and $a_{\mathrm{eq}}$, given by (see equation 72 )

$\frac{\delta_{\mathrm{eq}}}{\delta_{\mathrm{H}}} \approx 15 \ln \left(\frac{a_{\mathrm{eq}}}{a_{\mathrm{H}}}\right)$

where $\delta_{\mathrm{eq}} \equiv \delta_{k}\left(a_{\mathrm{eq}}\right)$ and $\delta_{\mathrm{H}} \equiv \delta_{k}\left(a_{\mathrm{H}}\right)$. Henceforth, we will omit the subscript $k$ when referring to the Fourier mode overdensity $\delta$. By $a_{\text {eq }}$, all modes of interest in forming galaxies and clusters had already entered the horizon, so they all experience the same factor of growth between $a_{\mathrm{eq}}$ and any later $a$ (i.e. for early times during the MD era, growth is the same as in an Einstein-de Sitter universe, in which case $\delta \propto a$ ).

It is convenient to describe the growth of density fluctuations in alternative dark matter models like SFDM in terms of their growth relative to that in CDM. For a range of wavenumbers, the growth of density fluctuations in SFDM with SI in the TF regime, for modes of interest to us in galaxy and large-scale structure formation, is the same after horizon crossing as for CDM. This is the case for modes of small enough wavenumber, as we shall see.

11 If we had chosen to define the mass associated with a horizon-filling mode as the mass inside a sphere of comoving radius $\lambda_{0}$, instead of $\lambda_{0} / 2$ as we did above, then the mass associated with the mode that enters at $a_{\text {eq }}$ would be 8 times larger than this, or $3 \times 10^{18} \mathrm{M}_{\odot}$. Alternatively, if we had, instead, defined the mass associated with a mode as just $\rho_{\mathrm{dm}, 0} \lambda_{0}^{3}$, rather than $4 \pi / 3 \rho_{\mathrm{dm}, 0}\left(\lambda_{0} / 2\right)^{3}$, as above, then the mass above, which is evaluated based upon the latter, should be multiplied by $6 / \pi \simeq 1.91$, instead, which would make $M_{\mathrm{eq}} \simeq 8 \times 10^{17} \mathrm{M}_{\odot}$. These are just matters of definition, of course, as the horizon-filling wavelength and the unperturbed mass associated with a given wavelength mode are not quantities of precise meaning, but we should try to compare different mode-associated masses using the same choice of definition.
There are two effects, however, that distinguish the two models. CDM behaves as a pressure-free, matter-like gas (with $w=0$ ) at all times, both during the RD era when it is a subdominant component of the total mass-energy density of the universe and during the MD era when it dominates that energy-density. SFDM differs in that regard. SFDM with repulsive SI is only nonrelativistic and matter-like (with $w=0$ ) at late times, after it transitions from being relativistic and radiation-like (with $w=1 / 3$ ). Although this transition is not instantaneous, it is fast enough that we can characterize it as a sudden transition at scale factor $a_{t}$. As we discuss below, this transition scale factor $a_{t}$ is set by the value of the ratio $\mathrm{g} / \mathrm{m}^{2}$. As pointed out by $\mathrm{Li}$ et al. (2014), observations of the CMB anisotropy constrain the allowed values of this ratio by requiring that the EOS of the background universe must transition from RD to MD at close to the same value of $a_{\mathrm{eq}}$ in both SFDM and CDM. This places an upper bound on the ratio, by requiring that $a_{t} \leq a_{\mathrm{eq}}$. As such, i.e. as long as $a_{t} \leq a_{\mathrm{eq}}$, modes that enter the horizon before $a_{t}$, when SFDMTF behaves like a radiation-like perfect fluid $(w=1 / 3)$, will not experience the logarithmic growth between $a_{\mathrm{H}}$ and $a_{t}$ that the same modes experience in CDM. In fact, these modes that enter the horizon prior to $a_{t}$ should evolve just like those of the dominant radiation component, which oscillate like sound waves of a relativistic gas with sound speed $c / \sqrt{3}$, without changing their amplitude. If so, then these modes that entered the horizon before $a_{t}$ and before $a_{\mathrm{eq}}$ would be deprived of the interval of logarithmic growth which the same modes would have experienced after horizon entry in CDM between $a_{\mathrm{H}}$ and $a_{\mathrm{eq}}$, according to equation (50). In the absence of CDM-like growth during this interval of scale factor, therefore, we might expect a net amount of growth for these modes over the entire $\mathrm{RD}$ era, including the time between $a_{t}$ and $a_{\text {eq }}$, equal to that which would result for CDM modes if we replaced $a_{\mathrm{H}}$ in their logarithmic growth factor in equation (50) by $a_{t}$.

There is a second effect, however, which may also interfere with the growth of SFDM modes, if their proper wavelength is below the Jeans length that results from the back reaction of SI pressure forces that act to cancel their gravitational instability. To be more specific, we must estimate this Jeans length and its dependence on scale factor, and consider which of these two effects dominates, for which modes, and during which intervals of scale factor.

Let us start by finding the transition scale factor $a_{t}$ and its dependence on SI strength $g$ and particle mass $m$. Suppose we identify the transition between $w=1 / 3$ and $w=0$ in equation (3), which goes through these values monotonically with increasing scale factor, by solving that equation for the scale factor at which $w=1 / 9$, assuming that, for small $w$, it is sufficient to take the homogeneous background value of $\left\langle|\phi|^{2}\right\rangle$ as given by $\left\langle|\phi|^{2}\right\rangle \simeq \rho_{\mathrm{dm}}=\rho_{\mathrm{dm}, 0} a^{-3}$, which then yields

$a_{1 / 9}=\left(3 \rho_{\mathrm{dm}, 0} \frac{g}{m^{2} c^{2}}\right)^{1 / 3}$

We can then write the scale factor for any $w$ as follows:

$a_{w}=a_{1 / 9}\left(\frac{1}{6 w}-\frac{1}{2}\right)^{1 / 3}$

In terms of $R_{\mathrm{TF}}$ in equation (21), we can write the transition scale factor as follows:

$$
\begin{aligned}
a_{1 / 9} & =\left(\frac{12 G \rho_{\mathrm{dm}, 0} R_{\mathrm{TF}}^{2}}{\pi c^{2}}\right)^{1 / 3} \\
& \simeq 1.8 \times 10^{-5}\left(\frac{\Omega_{\mathrm{dm}} h^{2}}{0.12}\right)^{2 / 3}\left(\frac{R_{\mathrm{TF}}}{1 \mathrm{kpc}}\right)^{2 / 3}
\end{aligned}
$$


If we now take $a_{t}=a_{1 / 9}$, then $a_{t}\left(R_{\mathrm{TF}}\right)$ follows

$a_{t}\left(R_{\mathrm{TF}}\right) \propto R_{\mathrm{TF}}^{2 / 3}$

In the radiation-dominated era, for which $H(a) \propto a^{-2}$, the mass that fills the Hubble volume is

$M_{\mathrm{H}, \mathrm{RD}}(a)=\frac{4 \pi}{3} \rho_{\mathrm{dm}, 0} a^{-3}\left(\frac{c}{H(a)}\right)^{3} \propto a^{3}$

which, evaluated at $a=a_{t}$ for a given value of $R_{\mathrm{TF}}$, is then

$M_{\mathrm{H}, \mathrm{RD}}\left(a_{t}\left(R_{\mathrm{TF}}\right)\right) \propto a_{t}^{3}\left(R_{\mathrm{TF}}\right) \propto R_{\mathrm{TF}}^{2}$

Combining this with the relationship above between $a_{t}$ and $R_{\mathrm{TF}}$, we can write the horizon-filling mass at the transition scale factor in terms of $R_{\mathrm{TF}}$ as

$M_{\mathrm{H}, \mathrm{RD}}\left(a_{t}\left(R_{\mathrm{TF}}\right)\right)=M_{\mathrm{H}, \mathrm{eq}}\left(\frac{R_{\mathrm{TF}}}{R_{\mathrm{TF}, \mathrm{eq}}}\right)^{2}$

where $R_{\mathrm{TF}, \mathrm{eq}}$ is the value that makes $a_{t}\left(R_{\mathrm{TF}, \mathrm{eq}}\right)=a_{\mathrm{eq}}$.

As discussed in Paper I, when $w \simeq 0$ (i.e. $a \gtrsim a_{t}$ ), the Jeans length scale below which gravitational instability is opposed by the backreaction of SI pressure is the proper wavelength $\lambda_{\mathrm{J}, 0}=2 R_{\mathrm{TF}}$ (where the subscript " 0 " refers to $w=0$ and the subscript "SI" is removed for simplicity but is implied). The associated SFDM-TF Jeans mass scale is given by the dark matter mass inside a sphere of radius $R_{\mathrm{TF}}$, at any scale factor $a$, according to

$$
\begin{aligned}
M_{\mathrm{J}, 0}(a) & =\frac{4 \pi}{3} \rho_{\mathrm{dm}, 0} a^{-3} R_{\mathrm{TF}}^{3} \\
& \simeq 4.6 \times 10^{12} \mathrm{M}_{\odot}\left(\frac{\Omega_{\mathrm{dm}} h^{2}}{0.12}\right)\left(\frac{a}{10^{-3.5}}\right)^{-3}\left(\frac{R_{\mathrm{TF}}}{1 \mathrm{kpc}}\right)^{3}
\end{aligned}
$$

For future reference, it will be useful to define the scale factor $a_{\mathrm{J}, 0}(M)$ at which the mass $M$ equals the Jeans mass, by inverting equation (58) to write

$$
\begin{aligned}
a_{\mathrm{J}, 0}(M) & =R_{\mathrm{TF}}\left(\frac{4 \pi \rho_{\mathrm{dm}, 0}}{3 M}\right)^{1 / 3}=\frac{R_{\mathrm{TF}} k}{\pi} \\
& \simeq 5 \times 10^{-4}\left(\frac{\Omega_{\mathrm{dm}} h^{2}}{0.12}\right)^{1 / 3}\left(\frac{M}{10^{12} \mathrm{M}_{\odot}}\right)^{-1 / 3}\left(\frac{R_{\mathrm{TF}}}{1 \mathrm{kpc}}\right)
\end{aligned}
$$

We are now in a position to compare this Jeans mass scale $M_{\mathrm{J}, 0}$ given above as a function of scale factor, for a given value of $R_{\mathrm{TF}}$, with the horizon-filling mass at each scale factor, to show the range of fluctuation mass-scales that are free of Jeans filtering from the moment those fluctuations re-enter the horizon during the RD era. For those fluctuation modes, CDM-like perturbation growth can be assumed to apply to the SFDM fluctuations, as well, as long as their horizon entry took place after SFDM transitions from a radiationlike to a matter-like EOS. We plot these quantities in Fig. 4 for 4 different values of $R_{\mathrm{TF}}=\{1000,100,10,1\} \mathrm{pc}$. As the figure indicates, all modes that enter the horizon super-Jeans (i.e. $M>M_{\mathrm{J}}$ when $M<M_{\mathrm{H}}$ ) do so when the SFDM EOS was matter-like. That means that the Jeans mass calculated above for the case when SFDM is matter-like is applicable, as we have assumed. Modes of smaller wavelength, for which horizon entry preceded the transition scale factor of the SFDM EOS, were relativistic and radiation-like upon entry, so their Jeans filtering during that phase should be calculated for a relativistic fluid, instead, because their effective sound speed is close to the speed of light and the Jeans scale is comparable to the horizon size, at which scale the Newtonian approximation breaks down.

Roughly speaking, when $w=1 / 3$ (i.e. $a \lesssim a_{t}$ ), the Jeans length is

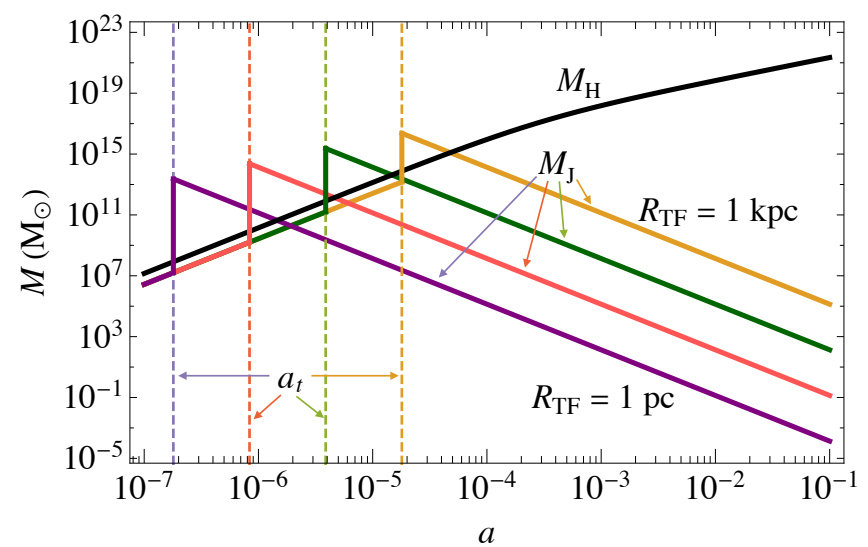

Figure 4. Cosmic evolution of the SFDM-TF Jeans-filter scale: The Incredible Shrinking Jeans Mass. The Jeans mass $M_{\mathrm{J}}\left(\right.$ i.e. $\left.M_{\mathrm{J}}=\frac{4 \pi}{3} \rho_{\mathrm{dm}, 0}\left(\pi / k_{\mathrm{J}}\right)^{3}\right)$ associated with the critical wavenumber $k_{\mathrm{J}}$ for linear density fluctuation modes in SFDM-TF that marks the boundary between growth (for $M>M_{\mathrm{J}}$ ) and oscillation (for $M<M_{\mathrm{J}}$ ), vs. scale factor $a$, for several illustrative values of the Thomas-Fermi radius $R_{\mathrm{TF}}$. Curve going from lower left to upper right is $M_{\mathrm{H}}(a)$, the mass that fills the horizon (i.e. the Hubble volume, of radius $c / H(a))$ at each scale factor. The diagonal curves from upper left to lower right represent the curves of $M_{\mathrm{J}}(a)$ for different values of the $R_{\mathrm{TF}}$ (1000, 100,10 , and $1 \mathrm{pc}$, in order from top to bottom). The dashed vertical lines and the discontinuities on each of these curves for different values of $R_{\mathrm{TF}}$ locates the value of the transition scale factor $a_{t}\left([1.8,8.4,39,180] \times 10^{-7}\right.$, in order from left to right) for the SFDM EOS, to the left of which $w=1 / 3$, while to the right $w=0$.

equal to the distance a relativistic sound wave can travel at the sound speed $c / \sqrt{3}$ in a Hubble time:

$R_{\mathrm{J}, 1 / 3}(a)=\frac{c}{H \sqrt{3}}$

The mass enclosed by this radius is the same as the mass inside the usual acoustic horizon during the RD era, which is just smaller than the mass inside the Hubble volume, according to

$$
\begin{aligned}
M_{\mathrm{J}, 1 / 3}(a) & =\frac{4 \pi}{3} \rho_{\mathrm{dm}, 0} a^{-3} R_{\mathrm{J}, \mathrm{rad}}^{3} \\
& \simeq 8.7 \times 10^{16} \mathrm{M}_{\odot}\left(\frac{\Omega_{\mathrm{dm}} h^{2}}{0.12}\right)\left(\frac{a}{10^{-3.5}}\right)^{3}
\end{aligned}
$$

independent of $R_{\mathrm{TF}}$, aside from the dependence of transition scale factor $a_{t}$ on it (see, for example, equation 54). This radiation-like Jeans mass defines the $a<a_{t}$ segment of each colored solid line in Fig. 4.

For any given mode, we can describe its cosmological evolution by drawing a horizontal line across the box in Fig. 4 at that mass scale and following it in time from small scale factor to large, for a fixed choice of SI strength, expressed by the value of $R_{\mathrm{TF}}$, as follows. At early times such that $a<a_{t}$, the SFDM-TF is radiationlike ( $w \approx 1 / 3)$, so its fluctuations behave during this phase just like those of fluctuations of the same wavenumber $k$ in the dominant radiation component. They are indistinguishable from the latter, in fact (i.e. Lagrangian volume changes in the scalar field and radiation are the same for these modes, during this phase of their evolution, so the fluctuation in dark matter mass density $\delta_{\text {SFDM }}$ for these modes is simply related to the energy-density fluctuation in the radiation $\delta_{\text {rad }}$ by $\left.\delta_{\text {SFDM }}=(3 / 4) \delta_{\text {rad }}\right)$. The smaller the value of $R_{\mathrm{TF}}$ is, the earlier this radiation-like phase of EOS will end. However, there is always a range of modes for which the mass is small enough 
(i.e. $k$ is large enough) to make horizon entry early enough that $a_{\mathrm{H}}<a_{t}$ for those modes, so they enter while the EOS of the scalar field is radiation-like. According to Fig. 4, these modes are sub-Jeans $\left(M<M_{\mathrm{J}, 1 / 3}\right)$ once they are within the horizon. Radiation fluctuations that enter the horizon during this epoch (which is RD), all start from the same $k$-independent amplitude when they are far outside the horizon, but oscillate like sound waves with constant amplitude after entry. This also describes the behavior of SFDM fluctuations of the same wavenumber, therefore, until the end of their radiation-like phase at $a=a_{t}$. At this time, the EOS of the scalar field transitions to matter-like $(w \approx 0)$ and, thereafter, the character of the evolution departs from that of the dominant radiation component, as described below.

There are three kinds of modes to track, as we follow their evolution after $a=a_{t}$, when the EOS turns matter-like. There are the smallermass modes, described above, that entered the horizon prior to this time. At the other extreme, there are modes that are so massive that they are matter-like and super-Jeans when they enter the horizon (i.e. $M>M_{\mathrm{J}, 0}$ when $M<M_{\mathrm{H}}$ ). In between these two mass ranges, there are modes which are matter-like and sub-Jeans upon horizon entry (i.e. $M<M_{\mathrm{J}, 0}$ when $M<M_{\mathrm{H}}$ ). In the first category, the high- $k$ modes that entered prior to this phase will still be sub-Jeans initially, after $a_{t}$, but with the Jeans mass now given by $M_{\mathrm{J}, 0}$, rather than $M_{\mathrm{J}, 1 / 3}$. Their fluctuations will transition from the radiation-like behavior described above to a new, matter-like behavior, thereafter. For the range of modes that entered the horizon after $a=a_{t}$, however, the EOS is matter-like at all times, thereafter. Their behavior once within the horizon depended upon whether they were above or below the Jeans mass $M_{\mathrm{J}, 0}$ at the time of their horizon entry. We expect perturbation modes that were super-Jeans upon horizon entry (i.e. modes with $M>M_{\mathrm{J}}$ when $M<M_{\mathrm{H}}$ ) during this phase to experience CDM-like growth, thereafter. For the intermediate-mass modes that were sub-Jeans $\left(M<M_{\mathrm{J}}\right)$ and matter-like $\left(w \approx 0\right.$ at $\left.a>a_{t}\right)$ when they were superhorizon $\left(M>M_{\mathrm{H}}\right)$, their evolution was also no different from that of CDM. After their horizon entry, however, these sub-Jeans modes oscillated like sound waves with an amplitude that grew as $a^{1 / 4}$, as described by Suárez \& Chavanis (2015) (see below). This sub-Jeans oscillatory behavior during the matter-like phase ( $a>$ $a_{t}$ ) of the scalar field, with amplitude growing as $a^{1 / 4}$, was also shared by the higher- $k$ modes that entered the horizon before $a=$ $a_{t}$, although these latter modes were already oscillating at constant amplitude prior to $a_{t}$, just like fluctuations in the dominant radiation component at that time.

The latter sub-Jeans behavior during the matter-like phase of SFDM-TF contrasts with the behavior of fluctuations in another matter component subject to Jeans-filtering by pressure forces, the baryons. For the baryons, subhorizon baryonic fluctuations below the Jeans scale in the CDM universe also oscillated like sound waves, but with an amplitude that actually decayed during the MD era, prior to their decoupling from photons. However, this sub-Jeans behavior of the baryonic component in which the amplitude does not grow but even decays prior to photon decoupling has a much smaller impact on structure formation in the standard CDM model than the corresponding sub-Jeans behavior of SFDM-TF described above, even though the latter involves oscillations that grow rather than decay. The reason is that the baryonic fluctuations are not required to "carry the load" as the driver of structure formation in the standard CDM model. In that model, subhorizon fluctuations in the dark matter were always able to grow. Hence, in that case, even though sub-Jeans baryonic fluctuations could not, the baryons were later able to catch up (after recombination) to those dark-matter fluctuations that had a head-start at growing during the RD era and beyond and never damped. On scales above the baryonic Jeans-filter mass after recombination, the CDM fluctuations were never filtered and so provided potential wells into which the baryons were able to fall, even if their own density fluctuations were initially negligible on these scales. Here, by contrast, SFDM is the dark matter (all of it, in fact), and, even though its Jeans-filtering bears some superficial similarity to the Jeans-filtering of baryonic fluctuations in CDM, there is no other component on which it can rely to drive its growth at later times. Hence, it is not possible for fluctuations in SFDM on these smaller scales to "re-animate" later, as those of the baryons did for the CDM model, by being driven to catch-up to perturbations in some other component that were able to grow while those in the SFDM did not.

That said, the Jeans mass in SFDM-TF decreases rapidly with time, so one might wonder whether this would free some fluctuation modes of their Jeans filtering after they transition from sub-Jeans to superJeans. The Jeans scale in the matter-like phase of SFDM corresponds to a fixed proper length, so it encompasses a smaller and smaller mass over time, scaling as it does with $a^{-3}$. As such, whatever a mode's amplitude is when it reaches this super-Jeans epoch following its sub-Jeans phase, it will be able to grow, thereafter, at the matter-like growth rate. It remains to be determined below, if that will be a significant effect, or, if not, if the growth of just those modes that were always super-Jeans from their horizon-entry epoch forward was sufficient on its own to form the full mass range of haloes at different redshifts required by astronomical observations.

\subsection{How the small-scale cut-off in the SFDM-TF transfer function depends on the polytrope radius}

The effect of Jeans-filtering described above is to introduce a smallscale cut-off in the transfer function for SFDM-TF, while for larger scales, the CDM and SFDM-TF transfer functions are the same. We will derive this effect in detail by solving the perturbation equations below. First, however, we will anticipate the result of those calculations by estimating the comoving wavenumber $k_{\text {cut }}$ and associated mass scale $M_{\text {cut }}$ at which the cut-off begins in the resulting transfer function, such that power is reduced relative to that in CDM (for $k \gtrsim k_{\text {cut }}$, or equivalently, $M \lesssim M_{\text {cut }}$ ), as follows. This cut-off will occur roughly at the wavenumber of the first mode to enter the horizon (i.e. the mode with the smallest $k$-value, corresponding to the largest mass scale) which is sub-Jeans when it enters. This is the mode for which $a_{\mathrm{J}, 0}=a_{\mathrm{H}}$, or equivalently, $R_{\mathrm{TF}}=c / H\left(a_{\mathrm{H}}\right)$ :

$R_{\mathrm{TF}}=\frac{c a_{\mathrm{H}}^{2}}{H_{0} \sqrt{\Omega_{\mathrm{rad}}}}=\frac{\pi^{2} H_{0} \sqrt{\Omega_{\mathrm{rad}}}}{c k_{\mathrm{cut}, \mathrm{TF}}^{2}}$

where $H\left(a_{\mathrm{H}}\right)$ is evaluated assuming all modes of interest enter the horizon when the Universe is $\mathrm{RD}$, and the last expression follows from our definition of $a_{\mathrm{H}}: c k / \pi=a_{\mathrm{H}} H\left(a_{\mathrm{H}}\right)$. Solving for $k_{\text {cut }}$ gives

$$
\begin{aligned}
k_{\text {cut }, \mathrm{TF}} & =\left(\frac{\pi^{2} H_{0} \sqrt{\Omega_{\mathrm{rad}}}}{c R_{\mathrm{TF}}}\right)^{1 / 2} \\
& \simeq 0.2 h \mathrm{Mpc}^{-1}\left(\frac{R_{\mathrm{TF}}}{1 \mathrm{kpc}}\right)^{-1 / 2} \\
M_{\mathrm{cut}, \mathrm{TF}} & =\frac{4 \pi}{3} \rho_{\mathrm{dm}, 0}\left(\frac{\pi}{k_{\mathrm{cut}, \mathrm{TF}}}\right)^{3} \\
& \simeq 1.4 \times 10^{15} \mathrm{M}_{\odot}\left(\frac{\Omega_{\mathrm{dm}} h^{2}}{0.12}\right)\left(\frac{R_{\mathrm{TF}}}{1 \mathrm{kpc}}\right)^{3 / 2}
\end{aligned}
$$

It will be useful to be able to compare the cut-off scale for SFDMTF to that which occurs when SI is not present and the SFDM model 
is in the FDM limit, instead. In the latter case, as we shall show below, there is a different Jeans mass which depends upon the particle mass $m$, rather than on the ratio $\mathrm{g} / \mathrm{m}^{2}$ involving SI, as it does for SFDM-TF. For FDM, the EOS is always matter-like during the galaxy-formation era (i.e. from horizon entry forward, for all modes that are relevant), and, just as for SFDM-TF and CDM, galaxy-formation modes enter the horizon when the Universe is RD. In that case, as we shall show in $\$ 4.3$, the Jeans mass and its dependence on scale factor are then given as follows:

$$
\begin{aligned}
& a_{\mathrm{J}, \mathrm{F}}=\frac{\hbar^{2} k^{4}}{16 \pi G \rho_{\mathrm{dm}, 0} m^{2}} \\
& \lambda_{\mathrm{J}, \mathrm{F}}=\left(\frac{\pi^{3} \hbar^{2}}{G \rho_{\mathrm{dm}, 0} a^{-3} m^{2}}\right)^{1 / 4} \\
& M_{\mathrm{J}, \mathrm{F}}(a)=\frac{4 \pi}{3} \rho_{\mathrm{dm}, 0} a^{-3}\left(\frac{\lambda_{\mathrm{J}, \mathrm{F}}}{2}\right)^{3} \\
& \quad \simeq 6.2 \times 10^{9} \mathrm{M}_{\odot}\left(\frac{\Omega_{\mathrm{dm}} h^{2}}{0.12}\right)^{1 / 4}\left(\frac{a}{10^{-3.5}}\right)^{-3 / 4} m_{22}^{-3 / 2}
\end{aligned}
$$

where $m_{22} \equiv m /\left(10^{-22} \mathrm{eV} / c^{2}\right)$. We can then do a similar calculation for FDM as we did above for SFDM-TF, to estimate the cut-off scale by setting $a_{\mathrm{J}, \mathrm{F}}=a_{\mathrm{H}}$, instead:

$$
\begin{gathered}
\frac{\hbar^{2} k_{\mathrm{cut}, \mathrm{F}}^{4}}{16 \pi G \rho_{\mathrm{dm}, 0} m^{2}}=\frac{c k_{\mathrm{cut}, \mathrm{F}} a_{\mathrm{H}}^{2}}{\pi H_{0} \sqrt{\Omega_{\mathrm{rad}}}}=\frac{\pi H_{0} \sqrt{\Omega_{\mathrm{rad}}}}{c k_{\mathrm{cut}, \mathrm{F}}} \\
k_{\mathrm{cut}, \mathrm{F}}=\left(\frac{16 \pi^{2} G H_{0} \rho_{\mathrm{dm}, 0} \sqrt{\Omega_{\mathrm{rad}}} m^{2}}{c \hbar^{2}}\right)^{1 / 5} \\
\simeq 3.9 h \mathrm{Mpc}^{-1}\left(\frac{\Omega_{\mathrm{dm}} h^{2}}{0.12}\right)^{1 / 5} m_{22}^{2 / 5}
\end{gathered}
$$

$$
\begin{aligned}
M_{\mathrm{cut}, \mathrm{F}} & =\frac{4 \pi}{3} \rho_{\mathrm{dm}, 0}\left(\frac{\pi}{k_{\mathrm{cut}, \mathrm{F}}}\right)^{3} \\
& \simeq 2.3 \times 10^{11} \mathrm{M}_{\odot}\left(\frac{\Omega_{\mathrm{dm}} h^{2}}{0.12}\right)^{2 / 5} m_{22}^{-6 / 5}
\end{aligned}
$$

Given this, we can equate equations (63) and (69) to find the equivalent FDM model that cuts off at the same scale as a given SFDM-TF model, in terms of $m$ and $R_{\mathrm{TF}}$. That is, the cut-off scale for a FDM model with a particle mass of $m_{22, \text { cut }}$ is the same as that for a SFDM-TF model with a TF radius of $R_{\mathrm{TF} \text {,cut }}$ given by

$R_{\mathrm{TF}, \mathrm{cut}} \simeq 3 \mathrm{pc}\left(\frac{\Omega_{\mathrm{dm}} h^{2}}{0.12}\right)^{-2 / 5} m_{22, \mathrm{cut}}^{-4 / 5}$

According to equation (71), the FDM model with particle mass $m_{22}=1$ results in suppression of small-scale structure relative to the CDM model below a cut-off scale that is comparable to the cut-off scale where suppression begins in the SFDM-TF regime if $R_{\mathrm{TF}} \approx 3 \mathrm{pc}$. This is interesting, since this fiducial value chosen for the FDM model is about the value required to flatten the density profiles of present-day dwarf galaxies relative to the CDM model, by making solitonic cores of radius equal to the de Broglie length there, of order $\sim 1 \mathrm{kpc}$. According to our results in Paper I and here in $\S 3$, however, when a halo forms in SFDM-TF, it has a core-envelope structure in which the envelope is CDM-like but the center flattens to the profile of the $(n=1)$-polytropic SI core, of radius $\simeq R_{\mathrm{TF}}$. As such, the value of $R_{\mathrm{TF}} \approx 3 \mathrm{pc}$, which our estimate above tells us will produce a cut-off of small-scale structure in the power-spectrum of density fluctuations relative to that for CDM, at a similar wavenumber to that for the FDM model when the latter has $m_{22}=1$, is very much smaller than that $\simeq 1 \mathrm{kpc}$ solitonic core size for the corresponding FDM model.

This estimate will guide our expectations from the detailed calculation of linear perturbation growth in the two models below. However, while it would be tempting to think we can use this estimate of the correspondence of the two models, with their parameters so chosen to place their small-scale cutoffs at the same wavenumber, the actual outcome of structure formation in the two models depends upon the full spectrum of perturbation modes and their cosmic evolution, down into the nonlinear era. A contrast between the two models may arise because the TF Jeans mass drops so much more rapidly with scale factor than does the FDM Jeans mass, for example, so scales that are Jeans-filtered in SFDM-TF at some epoch may be able to grow again at later times, while there is no such effect in FDM. Moreover, this effect of the "incredible shrinking Jeans mass" for SFDM-TF means that, while some structures may be underabundant relative to CDM as a result of their suppression in the linear perturbation stage - an effect which may be similar to that for the corresponding FDM model - the internal structures of haloes that result in the two models will differ considerably, if FDM solitonic cores in that model are much larger than the polytropic cores in the corresponding SFDM-TF haloes. The true comparison will require that we first derive the transfer function for the full spectrum of modes, which we do in the next section, before we can compare and distinguish the actual impact on galaxy and large-scale structure formation of the two models.

\subsection{Linear perturbations in SFDM-TF}

We are now ready to calculate the growth of linear perturbations for all modes of interest to us in the formation of galaxies and largescale structure, and from this, the transfer function for the SFDM-TF model, for comparison with those of the CDM and FDM models. We do this semi-analytically, by adopting several simplifying assumptions. The full treatment without approximation would require solving the relativistic Klein-Gordon-Einstein equations of motion for the scalar field, in a FLRW background universe, along with all other components of the standard cosmological model (i.e. radiation, baryons, neutrinos). This would be necessary to be able to consider the smooth entry of modes into the horizon, in general, from welloutside to deep within the horizon. This full treatment would also need to incorporate the non-standard expansion history which results from the evolution of the SFDM EOS, as described by Li et al. (2014, 2017), which depends upon the contribution of SFDM to the total energy density at each moment and, hence, depends on the value of particle parameters $m$ and $g / m^{2}$. In the most general case, the field can be either real or complex. In both cases, the homogeneous field can be described as a perfect fluid, which makes possible a fluid description for its evolution. However, when treating perturbations in the complex case, the perfect fluid description can break down in its stiff phase. We will, therefore, greatly facilitate the semi-analytical solution by the following approximations:

(1) We will assume that SFDM model parameters are constrained such that the structure formation era, when all modes of interest to us enter the horizon and evolve, is insignificantly affected by the "back-reaction" of the SFDM and its EOS evolution on the expansion history of the universe. It turns out that this is a very good approximation, since the departures from standard expansion history during this structure formation era are related 
to the transitions which occur between the EOS from matterlike $(w=0)$ to radiation-like $(w=1 / 3)$ and, thence, either to cosmological-constant-like $(w=-1)$ or stiff $(w=1)$, as one moves backward in time. The TF regime under consideration here ensures that the structure formation era is confined to the rapid oscillation phase of the scalar field, and the only transition that takes place during this period is that between radiation-like and matter-like. When that happens, as we said before, it must take place early enough that it does not perturb the value of $a_{\text {eq }}$ inferred from the measured CMB anisotropy. As pointed out in Li et al. (2014, 2017), this means there is an upper bound to the value of the SI strength in terms of $\mathrm{g} / \mathrm{m}^{2}$, and, hence, of the value of $R_{\mathrm{TF}}$. In particular, $g / m^{2} c^{4} \leq 4 \times 10^{-17} \mathrm{eV}^{-1} \mathrm{~cm}^{3}$ is required to satisfy $w\left(z_{\text {eq }}\right) \leq 0.001$, which is equivalent to requiring that $R_{\mathrm{TF}} \leq 5 \mathrm{kpc}$. When this bound is placed, there is a corresponding limitation placed on the contribution of the SFDM radiation-like energy density to the total during the RD era, which means that SFDM does not appreciably alter the expansion rate of the universe then.

(2) We will assume that SFDM behaves as a perfect fluid, so a fluid description is possible, with pressure and energy density related accordingly, by the EOS parameter in equation (3).

(3) We will assume that SFDM is radiation-like with $w=1 / 3$ at $a \leq a_{t}$ and matter-like with $w=0$ at $a>a_{t}$, i.e. we assume an instantaneous transition. We will take $a_{t}=a_{1 / 9}$.

(4) Once modes are well-within the horizon, we will treat them in the Newtonian approximation.

For each mode, we shall calculate as our reference solution, that for the growth of dark matter perturbations in the CDM model. The latter will be treated in the conventional way, as a nonrelativistic, collisionless, pressure-free gas (i.e. "dust"). An analytical solution is available for CDM perturbation modes when they are sub-horizon $\left(a \gg a_{\mathrm{H}}\right.$ ), given by (see, e.g., Mukhanov 2005, §6.3):

$$
\begin{aligned}
& \frac{\delta_{\mathrm{CDM}}(a)}{\delta_{\mathrm{H}}}=6\left[\ln \left(\frac{4 y}{x}\right)+\gamma_{\mathrm{E}}-\frac{7}{2}\right]\left(1+\frac{3 x}{2}\right) \\
& -6\left[\left(1+\frac{3 x}{2}\right) \ln \left(\frac{\sqrt{1+x}+1}{\sqrt{1+x}-1}\right)-3 \sqrt{1+x}\right] \\
& x \equiv \frac{a}{a_{\mathrm{eq}}} \\
& y \equiv \frac{c k a}{H_{0} \sqrt{3 \Omega_{\mathrm{rad}}}}
\end{aligned}
$$

where $\gamma_{\mathrm{E}} \simeq 0.577$ is the Euler-Mascheroni constant, and $\delta_{\mathrm{H}}$ is the asymptotic superhorizon value for the (mass) density contrast of a given mode. To match this analytical subhorizon solution in equation (72), valid when a mode is deep within the horizon, to the solution of a more exact, fully-relativistic, numerical solution that follows the perturbation smoothly as it enters the horizon from far outside to deep within, we define an effective horizon-entry scale factor for each mode, $a_{\mathrm{H} \text {,eff }}(k)$, that makes the two solutions agree when the modes are deep within the horizon. This is equivalent to our adopting

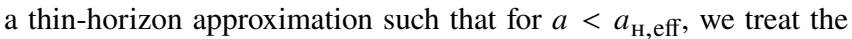
perturbations as frozen at their superhorizon values $\delta_{\mathrm{H}}$, while for $a>a_{\mathrm{H}, \text { eff }}$, the perturbations grow according to equation (72). To ensure that the density contrast is continuous across horizon-entry, then, we must choose $a_{\mathrm{H} \text {,eff }}$ such that, by equation (72),

$\delta_{\mathrm{CDM}}\left(a_{\mathrm{H}, \mathrm{eff}}\right)=\delta_{\mathrm{H}}$

for each mode $k$. For SFDM, as long as the scalar field is matterlike at this value of $a_{\mathrm{H} \text {,eff }}$ (i.e. $a_{\mathrm{H} \text {,eff }}>a_{t}$ ), we can also use this condition to set the horizon-entry value for an SFDM perturbation. That is, the superhorizon and horizon-entry values for a dark matter perturbation should be independent of the dark matter model (i.e. only their subhorizon evolution should differ):

$\delta_{\mathrm{SFDM}}\left(a_{\mathrm{H}, \mathrm{eff}}\right)=\delta_{\mathrm{CDM}}\left(a_{\mathrm{H}, \mathrm{eff}}\right)=\delta_{\mathrm{H}}$

(Henceforth, in $\$ 4.3$, the horizon-entry scale factor will refer to this $a_{\mathrm{H}, \mathrm{eff}}$, unless otherwise noted. Elsewhere, we refer more generally to "horizon-entry" in terms of a mode filling the Hubble volume, for which $k=k_{\mathrm{H}} \equiv \frac{\pi}{c} a_{\mathrm{H}} H\left(a_{\mathrm{H}}\right)$. The two definitions are quantitatively similar; we compare them in Appendix B, Fig. B1.)

For perturbations in SFDM-TF, there are two possible phases of behavior to consider, according to whether the scalar field is radiationlike or matter-like when a given mode is inside the horizon. Modes that enter the horizon when the scalar field is radiation-like (i.e. with $a_{\mathrm{H} \text {,eff }} \leq a_{t}$ ) evolve just like those in the radiation component that dominates the total energy density. Since we will only consider models for which the field transitions from radiation-like to matterlike at a scale factor $a_{t}<a_{\mathrm{eq}}$, the field will behave like radiation in a radiation-dominated universe for $a<a_{t}$, which has a known analytical solution, given by (see, e.g., Mukhanov 2005, §7.3):

$\frac{\delta_{\text {rad }}(a)}{\delta_{\text {rad }}(0)}=-3\left[\left(\frac{2-y^{2}}{y^{2}}\right)(\operatorname{sinc} y-\cos y)-\operatorname{sinc} y\right]$

where sinc $y \equiv \sin y / y$. According to this solution, such modes oscillate like sound waves in a relativistic fluid with sound speed $c / \sqrt{3}$, with constant amplitude. This analytical solution is fully-relativistic and properly follows the perturbations from far outside the horizon through horizon entry, so the time at which oscillations begin as a function of wavenumber is not arbitrary, but reflects the synchronization of horizon entry for all modes ${ }^{12}$ of a given $k$.

For modes that are inside the horizon when the scalar field is matter-like, instead (i.e. whenever $a>\max \left[a_{\mathrm{H}, \mathrm{eff}}, a_{t}\right]$ ), each mode evolves thereafter according to the following differential equation (Suárez \& Matos 2011; Chavanis 2012):

$\ddot{\delta}+2 H \dot{\delta}+\left(\frac{\hbar^{2} k^{4}}{4 m^{2} a^{4}}+\frac{\bar{c}_{s, \mathrm{SI}}^{2} k^{2}}{a^{2}}-4 \pi G \rho_{\mathrm{dm}, 0} a^{-3}\right) \delta=0$

where overdots denote differentiation with respect to cosmic time $t$, and $\bar{c}_{s, \text { SI }}$ is the sound speed of the background associated with SI pressure, given by:

$\bar{c}_{s, \mathrm{SI}}^{2}=\frac{g}{m^{2}} \rho_{\mathrm{dm}, 0} a^{-3}$

The first term inside the parentheses in equation (78) is the quantum pressure term, while the second is the effect of SI pressure. The third term is responsible for gravitational growth. The negative sign in front of that third term means that the first two, both positive-definite, act to oppose gravitational growth. The sum of all three terms inside the parentheses can be zero for a certain value of wavenumber, which we shall call the generalized Jeans wavenumber, $k_{\mathrm{J}}$. Modes with wavenumber $k<k_{\mathrm{J}}$ are able to grow, while those with $k>k_{\mathrm{J}}$ oscillate like sound waves for which the evolution of their amplitude depends upon which of those first two terms dominates. We note that the scale factor dependences of those two first terms are different, so, in principle, if one term dominates at some time, the other may dominate at other times.

12 Our only approximation in this case is that, in practice, we assign $a_{t}$ as the scale factor at which the average EOS parameter $w$ for the scalar field equals $1 / 9$, and assume that SFDM instantaneously transitions from radiation-like to matter-like at $a_{t}$. 
In the TF regime, the regime of primary interest to us here, we can take the limit in which $\hbar k^{2} / H m \rightarrow 0$ in equation (78) above and drop the first term inside the parentheses. This means that, in this linear regime, SI dominates and quantum pressure is unimportant. This contrasts the linear perturbation regime with the nonlinear regime in which inhomogeneities even on the scale of the de Broglie wavelength (and their contribution to quantum pressure) cannot be neglected, since they are responsible for transporting momentum and, thereby, affecting larger scales, even in a spatially-averaged description, as we discussed in Paper I. In this linear regime, then, subhorizon perturbations in SFDM-TF during its matter-like phase obey the following equation:

$\ddot{\delta}+2 H \dot{\delta}+\left(\frac{\bar{c}_{s, \mathrm{SI}}^{2} k^{2}}{a^{2}}-4 \pi G \rho_{\mathrm{dm}, 0} a^{-3}\right) \delta=0$

This equation is the origin of our expression for the proper TF Jeans wavelength $\lambda_{\mathrm{J}, 0}$ in $\S 4.1$, which we obtain by solving for the comoving Jeans wavenumber $\left(k_{\mathrm{J}, 0}=2 \pi a / \lambda_{\mathrm{J}, 0}\right)$ that makes the terms inside the parentheses in equation (80) sum to zero:

$k_{\mathrm{J}, 0}=a \sqrt{\frac{4 \pi G m^{2}}{g}}=\frac{\pi a}{R_{\mathrm{TF}}}$

According to equations (78) and (79), if we are in the TF regime at the end of the evolution period of interest, then we were always in the TF regime at all earlier times, as well, so equation (80) remains valid throughout this phase of matter-like EOS.

In practice, we solve equation (80) for $\delta \equiv \delta_{\mathrm{SFDM}}(a) / \delta_{\mathrm{H}}$. The remaining uncertainty is then about what initial condition to impose on the solution. There are two regimes:

(i) $a_{\mathrm{H}, \mathrm{eff}}>a_{t}-$ modes enter the horizon when the field is matterlike:

In this case, since all dark matter models should behave the same for superhorizon modes, we use the CDM horizon entry amplitude and slope

$$
\begin{aligned}
& \delta_{\mathrm{SFDM}}\left(a_{\mathrm{H}, \mathrm{eff}}\right)=\delta_{\mathrm{CDM}}\left(a_{\mathrm{H}, \mathrm{eff}}\right) \\
& \delta_{\mathrm{SFDM}}^{\prime}\left(a_{\mathrm{H}, \mathrm{eff}}\right)=\delta_{\mathrm{CDM}}^{\prime}\left(a_{\mathrm{H}, \mathrm{eff}}\right)
\end{aligned}
$$

or, equivalently,

$$
\begin{aligned}
& \delta\left(a_{\mathrm{H}, \mathrm{eff}}\right)=1 \\
& \delta^{\prime}\left(a_{\mathrm{H}, \mathrm{eff}}\right)=\delta_{\mathrm{CDM}}^{\prime}\left(a_{\mathrm{H}, \mathrm{eff}}\right) / \delta_{\mathrm{H}}
\end{aligned}
$$

(ii) $a_{\mathrm{H}, \mathrm{eff}} \leq a_{t}$-modes enter the horizon when the field is radiationlike:

In this case, since SFDM should behave like radiation prior to $a_{t}$, we use the (normalized) amplitude and slope of the radiation solution at $a_{t}$

$$
\begin{aligned}
& \delta\left(a_{t}\right)=\delta_{\mathrm{rad}}\left(a_{t}\right) / \delta_{\mathrm{rad}}(0) \\
& \delta^{\prime}\left(a_{t}\right)=\delta_{\mathrm{rad}}^{\prime}\left(a_{t}\right) / \delta_{\mathrm{rad}}(0)
\end{aligned}
$$

\subsection{The transfer function: SFDM-TF vs. CDM}

It is customary to describe the growth of density fluctuations in alternative dark matter models to CDM in terms of a normalized transfer function, defined as follows:

$$
\begin{aligned}
T_{\mathrm{SFDM}}^{2}(k, a) & \equiv \frac{\mathcal{T}_{\mathrm{SFDM}, k}^{2}\left(a, a_{i}\right)}{\mathcal{T}_{\mathrm{CDM}, k}^{2}\left(a, a_{i}\right)}=\frac{P_{\mathrm{SFDM}}(k, a)}{P_{\mathrm{CDM}}(k, a)} \\
& =\left|\frac{\delta_{\mathrm{SFDM}}(k, a)}{\delta_{\mathrm{CDM}}(k, a)}\right|^{2}
\end{aligned}
$$

(see also equation 42), where $P(k, a)$ is the linear-fluctuation power spectrum of the dark matter for each model, as labeled.

\subsection{Comparison with FDM}

As a proof of principle, we can also compute the transfer function for the FDM model, using a procedure similar to that outlined above for SFDM-TF, and compare our results to those of the publicly-available code AXIONCAMB ${ }^{13}$ by Hlozek et al. (2015). This code is presumably more accurate since it solves the fully-relativistic perturbation equations numerically, from superhorizon through subhorizon regimes for each $k$, including their coupling to fluctuations in other components of energy-density, as well. To derive the transfer function for FDM, we drop the SI-term in equation (78):

$\ddot{\delta}+2 H \dot{\delta}+\left(\frac{\hbar^{2} k^{4}}{4 m^{2} a^{4}}-4 \pi G \rho_{\mathrm{dm}, 0} a^{-3}\right) \delta=0$

This equation for self-gravitating perturbations in the free-field (noSI) case was also discussed elsewhere, e.g. Khlopov, Malomed \& Zeldovich (1985). Once again, we obtain the Jeans wavelength and wavenumber by setting the terms in parentheses equal to zero:

$k_{\mathrm{J}, \mathrm{F}}=\left(\frac{16 \pi G \rho_{\mathrm{dm}, 0} m^{2} a}{\hbar^{2}}\right)^{1 / 4}$

There are three noteworthy distinctions between the FDM and SFDM-TF models with regards to the solutions of their linear perturbation equations:

(1) when an FDM mode is sub-Jeans, it oscillates with a constant amplitude, whereas a sub-Jeans SFDM-TF mode oscillates with an amplitude that grows like $a^{1 / 4}$;

(2) the FDM Jeans mass shrinks much more slowly over time than that for SFDM-TF $\left(M_{\mathrm{J}, \mathrm{F}} \propto a^{-3 / 4}\right.$ vs. $\left.M_{\mathrm{J}, 0} \propto a^{-3}\right)$, so sub-Jeans modes of SFDM-TF can become super-Jeans sooner and with more time left to grow like CDM; and

(3) in the FDM limit, the radiation-like phase is absent, so the initial conditions are set by equations (82) - (85) for all modes.

The first two of these distinctions explain why FDM has a sharper cut-off in the transfer function than SFDM-TF does. ${ }^{14}$

\subsection{The Halo Mass Function}

The linear transfer function for the SFDM-TF model computed above can be used to calculate the halo mass function ("HMF") - defined

13 https://github.com/dgrin1/axionCAMB

14 The sharp cut-off in the transfer function for FDM was also discussed by $\mathrm{Hu}$, Barkana \& Gruzinov (2000), an important early contribution to this subject. They reported an approximate fitting formula for the solution for different wavenumbers $k$ at a given scale factor, parameterized in terms of the ratio of that wavenumber to the Jeans wavenumber at that scale factor, $k / k_{\mathrm{J}}$, which differs somewhat from the solutions presented here, which are numerical solutions of equation (89). While they do not give the linear perturbation equations they solved numerically, they say they treated FDM as a perfect fluid with an effective sound speed, which, in its rapid-oscillation phase of matter-like EOS, is given by $c_{S, \mathrm{~F}}^{2}=\hbar^{2} k^{2} /\left(4 m^{2} a^{2}\right)$. If so, then the dependence of the Jeans wavenumber on scale factor should agree with ours derived by setting the bracket in our equation (89) to zero, namely $k_{\mathrm{J}} \propto a^{1 / 4}$, in both the RD and MD eras. While agreeing with this for the MD era, however, Hu et al. (2000) states that $k_{\mathrm{J}}$ is independent of $a$ during the important $\mathrm{RD}$ era, which is not correct. 
as the comoving number density of haloes per unit logarithm of halo mass $M, \frac{d n}{d \ln M}$. There is an extensive literature on the various choices for analytical and empirical prescriptions for computing the HMF from the transfer function so as to predict the results of cosmological N-body simulation and their analysis by different halofinding algorithms (see, e.g., Knebe et al. 2013; Diemer 2018, and references therein). As our purpose here is to compare and contrast the HMF of the SFDM-TF model with those of CDM and FDM, and there are not yet $\mathrm{N}$-body results for the former with which to perform this comparison (and hardly any for FDM, either), we will adopt the most straightforward prescription for calculating the HMFs semi-analytically for all three models, from their respective linear power spectra $P(k, a)$, using the standard Press-Schechter ("PS") formalism (Press \& Schechter 1974). The latter is based upon the Gaussian statistics of the initial density perturbations, smoothed on a given mass scale, and the ansatz that a given local overdensity in the smoothed, linearly-perturbed density field will collapse and virialize at the time predicted for a spherical top-hat perturbation of that initial overdensity to reach infinite density. A more rigorous underpinning for this remarkably successful ansatz was subsequently provided by the Excursion Set Theory of Bond et al. (1991), including derivation of the PS formula for the HMF for the case of "sharp $k$-space filtering", about which we will say more below.

The PS HMF depends, not only on the underlying power spectrum of the initial, Gaussian-random density fluctuations, but also on the shape of the function adopted to smooth the density field. The purpose of this smoothing function is to squelch high-frequency modes that correspond to fluctuations of wavelength smaller than that which encompasses a given halo mass scale $M$, on average. The mass variance $\sigma_{\mathrm{M}}^{2}(M, a)$ of the linear overdensity field smoothed in coordinate space with a spherical window function $W(\boldsymbol{x}, R)$ - where $R$ is the comoving radius of a sphere in the unperturbed background universe which encompasses the mass $M, R=\left[M /\left(\frac{4 \pi}{3} \rho_{\mathrm{dm}, 0}\right)\right]^{1 / 3}$ - is related to the power spectrum $P(k, a)$ and the Fourier transform of this window function, $\mathcal{W}(k, R)$, as follows:

$\sigma_{\mathrm{M}}^{2}(M, a)=\frac{1}{2 \pi^{2}} \int_{0}^{\infty} P(k, a) \mathcal{W}(k, R) k^{2} d k$

The window function is usually taken to be a top-hat filter, expressed in Fourier space as

$\mathcal{W}_{\text {top-hat }}(k, R)=3 \frac{\sin (k R)-k R \cos (k R)}{(k R)^{3}}$

However, for models with sharp cut-offs in the power spectrum such as FDM, a sharp- $k$ filter is preferred in order to have the cut-off reflected in the HMF (Kulkarni \& Ostriker 2020):

$\mathcal{W}_{\text {sharp- } k}(k, R)= \begin{cases}1 & k<\alpha / R \\ 0 & k>\alpha / R\end{cases}$

where $\alpha$ is a free parameter which we set to 1.9 in order to make the resulting HMF agree with that computed using the top-hat filter at large mass. The PS HMF is then given by

$\frac{d n}{d \ln M}=-\sqrt{\frac{2}{\pi}} \frac{\rho_{\mathrm{dm}, 0}}{M} \frac{\delta_{c}}{\sigma_{\mathrm{M}}} e^{-\delta_{c}^{2} / 2 \sigma_{\mathrm{M}}^{2}} \frac{d \ln \sigma_{\mathrm{M}}}{d \ln M}$

where $\delta_{c}=1.686$ is the critical linear overdensity for halo formation derived from the spherical top-hat collapse model. ${ }^{15}$

15 Kulkarni \& Ostriker (2020) used $\alpha=2.5$ following the work of Benson et al. (2013), who found that value to be a good fit to warm dark matter simulations, and re-scaled $\delta_{c}$ by a factor of 1.195 in order to produce a
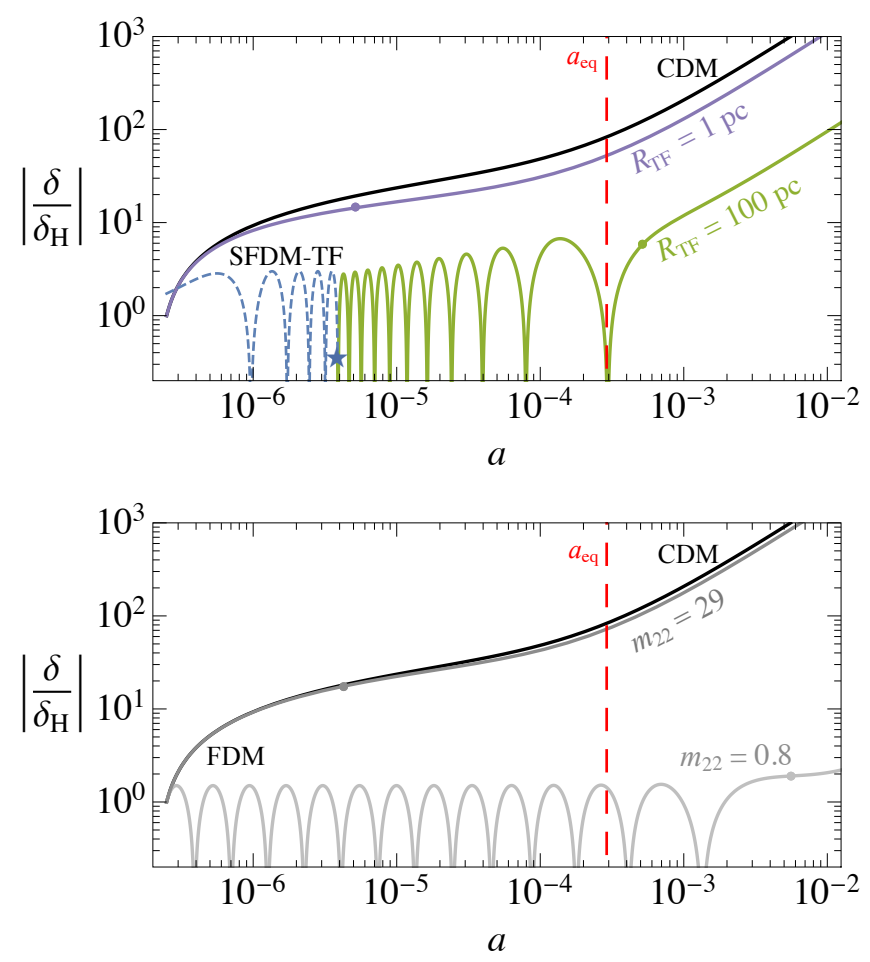

Figure 5. Linear perturbation growth after horizon entry, for a single Fourier mode. Solutions of the differential equations for the evolution of linear-perturbation fractional overdensity $\left|\delta / \delta_{\mathrm{H}}\right|$ vs. scale factor $a$ are plotted for a single Fourier mode, with wavenumber $k$ associated with a mass $M=$ $10^{9} \mathrm{M}_{\odot}\left(\right.$ where $M=\frac{4 \pi}{3} \rho_{\mathrm{dm}, 0}(\pi / k)^{3}$ and $\left.k \simeq 16 \mathrm{Mpc}^{-1}\right)$ in the SFDMTF (top panel) and FDM (bottom panel) models, for two different cases for each. The purple and green SFDM-TF lines correspond to $R_{\mathrm{TF}}=1 \mathrm{pc}$ and $R_{\mathrm{TF}}=100 \mathrm{pc}$, respectively. The light and dark grey FDM lines correspond to $m_{22}=0.8$ and $m_{22}=29$, respectively. The black line is the CDM solution. Dots of the same color as the curves on which they reside mark the Jeans scale factors, $a_{\mathrm{J}, 0}$ (SFDM-TF) and $a_{\mathrm{J}, \mathrm{F}}(\mathrm{FDM})$. The blue dashed line in the top panel shows the SFDM-TF solution for $R_{\mathrm{TF}}=100 \mathrm{pc}$ when the scalar field is radiation-like (i.e. $w=1 / 3$ ) (given by equation 77), and the blue star marks the transition scale factor $a_{t}$ for this case, after which SFDM-TF is matter-like (green solid line). The red dashed lines in both panels marks radiation-matter equality.

\subsection{Results of linear perturbation theory: SFDM-TF vs. CDM vs. FDM}

\subsubsection{Perturbation growth for a single mode}

In Fig. 5, we plot solutions of the differential equations described in $\S 4.3$ for the fractional linear overdensity $\left|\delta / \delta_{\mathrm{H}}\right|$ vs. scale factor $a$, for the subhorizon evolution of a single, representative Fourier mode $k$. We compare the analytical solution for this mode in the CDM model with our solutions for SFDM-TF and FDM, for illustrative cases with different parameters for the two kinds of SFDM models. The wavenumber $k$ for our representative mode is that associated with a

sharp- $k$ filtered HMF that was consistent with the top-hat filtered HMF at large mass. In view of the fact that we are here comparing the HMF's of CDM, SFDM-TF and FDM, these choices would not be appropriate for all three models, especially in view of the strong differences we find between SFDM-TF and FDM. However, we also find that adopting these choices would not affect our general conclusions. 
mass $M=10^{9} \mathrm{M}_{\odot}\left(\right.$ where $\left.M=(4 \pi / 3) \rho_{\mathrm{dm}, 0}(\pi / k)^{3}\right)$. For SFDM$\mathrm{TF}$, we present two cases, with $R_{\mathrm{TF}}=1$ and $100 \mathrm{pc}$, respectively. For FDM, we present two cases as well, with values of the particle mass $m_{22}=0.8$ and 29 , respectively.

The perturbation evolution for this mode in pressure-free CDM plotted in Fig. 5 shows the familiar transition from RD-era logarithmic growth to the MD-era growth rate $(\delta \propto a)$. This transition for CDM is gradual, but is centered around $a=a_{\text {eq }}$. By contrast, the perturbation evolution for both the SFDM-TF and FDM solutions plotted in Fig. 5 shows the effects of pressure and, hence, Jeans filtering, at early times. The evolution changes when this mode passes from sub-Jeans $\left(k>k_{\mathrm{J}}\right)$ to super-Jeans $\left(k<k_{\mathrm{J}}\right)$ at $a=a_{\mathrm{J}}$, where the values of $a_{\mathrm{J}}$ (marked by a dot on each curve) depend upon the model parameters and differ for each case. When a mode of either SFDMTF or FDM is sub-Jeans, its fractional overdensity oscillates like a sound wave. As such, the periods of oscillation in the two models are inversely proportional to their effective sound speeds. For SFDM-TF, this sound speed increases with $R_{\mathrm{TF}}$ when the EOS is matter-like, but is a constant, independent of $R_{\mathrm{TF}}$, when the EOS is radiationlike. The scale factor $a_{t}$ at which this transition from radiation-like to matter-like EOS occurs for SFDM-TF also depends on $R_{\mathrm{TF}}$, with $a_{t}$ increasing with $R_{\mathrm{TF}}$. For FDM, which is always matter-like during the era we study here, the sound speed decreases with increasing $m_{22}$. These points allow us to understand the behavior of the different curves in Fig. 5, as follows.

Consider, first, the curves for $m_{22}=0.8$ and $R_{\mathrm{TF}}=100 \mathrm{pc}$, respectively. For these values of $R_{\mathrm{TF}}$ and $m_{22}$, the periods of subJeans oscillation are smaller than a Hubble time during that phase of evolution. In that case, their fractional overdensities oscillate rapidly enough to pass from positive to negative and back again a number of times, so the curves of their absolute value displayed in Fig. 5 appear like trajectories of a bouncing ball. For FDM, the sub-Jeans oscillations have a constant amplitude, whereas, when the sub-Jeans SFDM-TF mode oscillates, its amplitude is initially constant for a time (the blue-dashed portion), during its radiation-like phase, but then grows like $a^{1 / 4}$ after it transitions at $a=a_{t}$ (as marked on the curve) to a matter-like EOS. In both cases, the oscillations cease near $a \approx a_{\mathrm{J}}$, and perturbation growth rates become CDM-like for $a \gg a_{\mathrm{J}}$.

In the case of the other two curves in Fig. 5, that for SFDM-TF with $R_{\mathrm{TF}}=1 \mathrm{pc}$ and FDM with $m_{22}=29$, the oscillatory behavior during their sub-Jeans phase is apparently absent, but this reflects the fact that the change of model parameters has pushed their sound speeds to be smaller and their oscillation "periods" to be larger than a Hubble time. In that case, the background universe is expanding too fast for the density to complete a single oscillation. These cases therefore follow the CDM trajectory fairly closely, albeit with their growth slightly hampered due to the brief initial sub-Jeans phase.

\subsubsection{Normalized transfer functions}

In Figs. 6(a) and (b), our solutions for linear perturbation growth for different $k$-modes are used to plot the normalized transfer functions $T_{\mathrm{SFDM}}^{2}(k, a)$ for SFDM-TF vs. $k$, for several illustrative cases, with $R_{\mathrm{TF}}=1000,100,10$, and $1 \mathrm{pc}$ (left to right). For comparison, we also plot FDM transfer functions $T_{\mathrm{FDM}}^{2}(k, a)$, for the same two masses, $m_{22}=0.8$ and 29, as in Fig. 5 above. In Fig. 6(a), transfer functions for FDM were generated by AXIONCAMB, while in Fig. 6(c), we compare those with our solutions for FDM, to show their close agreement. This agreement serves to validate our solutions for both FDM and SFDM-TF, since the former were derived by the same procedure as for our SFDM-TF solutions. Dots locate values of $k_{\text {cut }}$ derived in
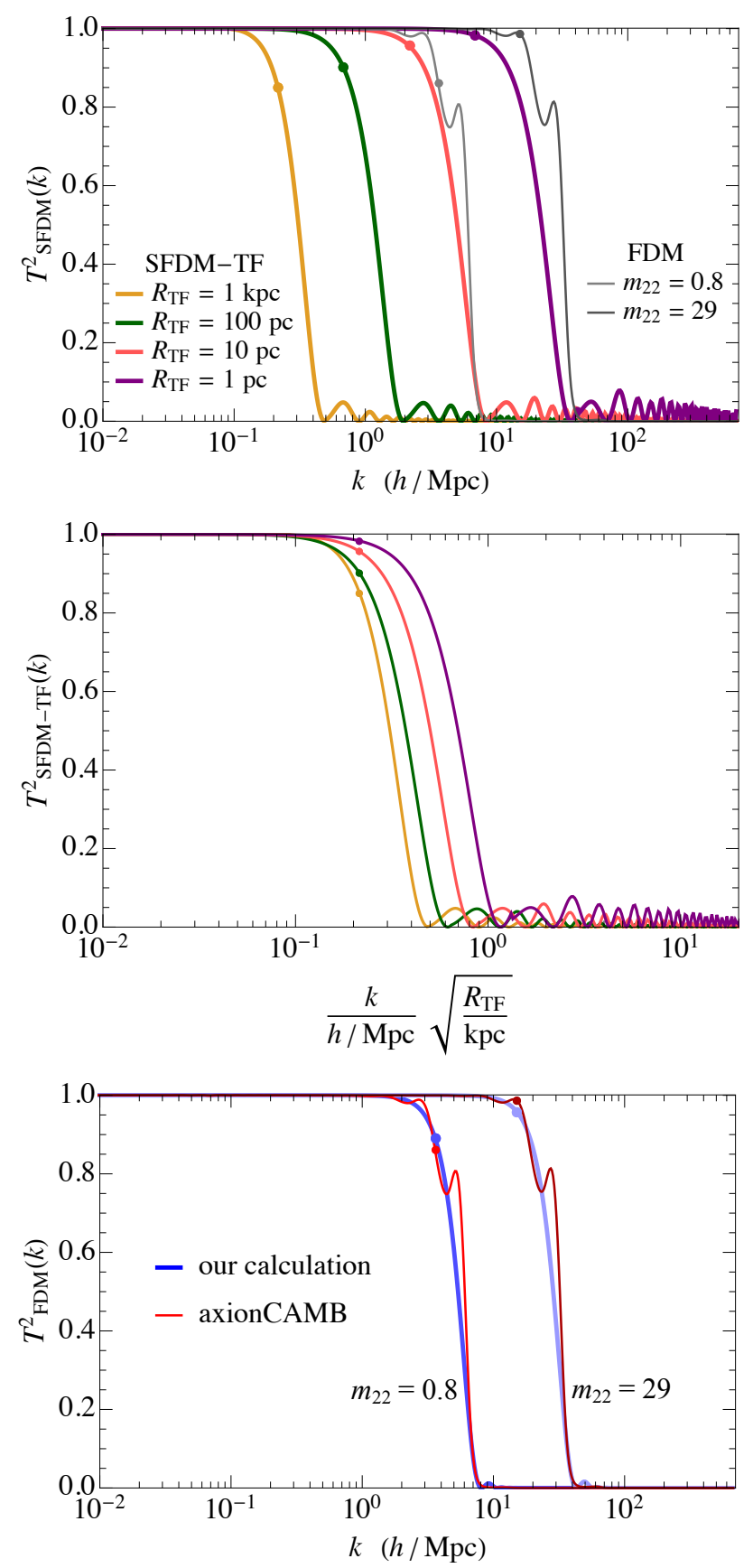

Figure 6. Linear transfer functions. (a) (top panel) Normalized transfer functions $T_{\mathrm{SFDM}}^{2}(k, a)$ for SFDM-TF vs. comoving wavenumber $k$, for $R_{\mathrm{TF}}=$ $\{1000,100,10,1\} \mathrm{pc}$ (left to right). For comparison, we plot FDM transfer functions $T_{\mathrm{FDM}}^{2}(k, a)$ for $m_{22}=\{0.8,29\}$, generated by АХIONCAMB. Dots locate values of $k_{\text {cut }}$ derived in $\$ 4.2$ to estimate the cut-off wavenumber above which high-frequency modes are suppressed relative to CDM. (b) (middle panel) SFDM-TF transfer functions in (a), but with $k$-axis rescaled by dependence of $k_{\text {cut }}$ on $R_{\mathrm{TF}}$, so that locations on the horizontal axis at which $k=k_{\text {cut }}$ are the same for all $R_{\mathrm{TF}}$. SFDM-TF curves for larger $R_{\mathrm{TF}}$ start to deviate from CDM more sharply than for smaller $R_{\mathrm{TF}}$, so $T_{\mathrm{SFDM}}^{2}\left(k_{\mathrm{cut}}\right)$ is closer to 1 for smaller $R_{\mathrm{TF}}$. (c) (bottom panel) FDM transfer functions in (a) (light and dark red lines), generated by АХIONCAMB, are compared to our solutions of subhorizon linear perturbation growth for FDM (dark and light blue), i.e. same procedure we used for SFDM-TF, but using the FDM linear perturbation equation (89), instead. 
$\$ 4.2$ to estimate the cut-off wavenumber above which high-frequency modes are suppressed relative to CDM.

In Fig. 6(b) (the middle panel), we replot the SFDM-TF transfer functions in Fig. 6(a) (the top panel) with the $k$-axis rescaled by the dependence of $k_{\text {cut }}$ on $R_{\mathrm{TF}}$, so that locations on the horizontal axis at which $k=k_{\text {cut }}$ are the same for all $R_{\mathrm{TF}}$. This shows that the smaller the value of $R_{\mathrm{TF}}$ is, the more gradual is the decline relative to CDM at wavenumbers where the SFDM-TF transfer function begins to decline relative to CDM at high $k$. In addition, since curves for larger $R_{\mathrm{TF}}$ start to deviate from CDM more sharply than for smaller $R_{\mathrm{TF}}$, $T_{\mathrm{SFDM}}^{2}\left(k_{\mathrm{cut}}\right)$ is closer to 1 for smaller $R_{\mathrm{TF}}$. We can also see from this figure that the amplitudes of the oscillations at high wavenumber are smaller for larger $R_{\mathrm{TF}}$. This may be due, in part, to the fact that, for larger $R_{\mathrm{TF}}$, modes with $k \gg k_{\text {cut }}$ have more of their subJeans evolution occur during the matter-dominated era $\left(a>a_{\mathrm{eq}}\right)$. Since CDM modes grow faster during this era (linearly with scale factor, as opposed to logarithmically for $a<a_{\text {eq }}$ ), the $k \gg k_{\text {cut }}$ SFDM-TF modes for larger $R_{\mathrm{TF}}$ cases "miss out" on more of this comparatively rapid, linear growth phase than the corresponding modes of the smaller $R_{\mathrm{TF}}$ cases, and so are more suppressed relative to $\mathrm{CDM}$.

According to our analytical estimates in $\$ 4.1$, it is possible to choose values of $R_{\mathrm{TF}}$ for SFDM-TF and $m_{22}$ for FDM so as to facilitate their direct comparison, by selecting values that make the small-scale cut-offs in their respective transfer functions occur at roughly the same wavenumber (see equation 71). In that case, for example, our solution for FDM with $m_{22}=0.8$ can be compared with that for SFDM-TF with $R_{\mathrm{TF}} \simeq 10 \mathrm{pc}$. In this way, observational constraints on FDM can also serve as a rough proxy for constraints on SFDM-TF, since both models have perturbation growth which is CDM-like at small $k$ but suppressed at high $k$, i.e. values of $k$ above the cut-off.

This estimator for the $R_{\mathrm{TF}}$-values that make the transfer function cut-offs for SFDM-TF and FDM for a given $m_{22}$ "align" in wavenumber by solving equation (71) should be used with caution, however. The shapes of their transfer functions below the cut-off wavenumber differ somewhat. As a result, if different observables measure the amount of structure on different scales, i.e. at different $k$, then the correspondence between the $m_{22}$ - and $R_{\mathrm{TF}}$-values that make the two model predictions agree may depend upon which observable is used to constrain them. As an example, the two models can have very different half-power wavenumbers, $k_{1 / 2}$ in their transfer functions, even when their $k_{\text {cut }}$-values are identical.

The two illustrative values of FDM particle mass we chose for Figs. 5 and 6 will appear again in later figures, too, in which we compare the SFDM-TF and FDM models further. These values roughly span the current range of constraints in the FDM literature, placed by various observational probes, namely, $m_{22}=0.8$ (e.g. fiducial case of Robles et al. 2019 and Paper I) and $m_{22}=29$ (e.g. lower bound from Nadler et al. 2021). For these values of $m_{22}$, de Broglie wavelengths inside a present-day dwarf galaxy of mass $10^{10} \mathrm{M}_{\odot}$ with velocity dispersion $\sigma_{\mathrm{v}} \approx 30 \mathrm{~km} \mathrm{~s}^{-1}$, for example, are $\approx 4 \mathrm{kpc}$ and $0.1 \mathrm{kpc}$, respectively, so haloes of this mass that formed in FDM would have solitonic cores of these sizes. If we chose $R_{\mathrm{TF}}$-values of this size, so as to make the SI-polytropic cores inside SFDM-TF haloes as large as this, then the $k_{\text {cut }}$-values for SFDM-TF will be much smaller than the $k_{\text {cut }}$-values for these FDM models. The transfer function for SFDM-TF in that case would begin to cut-off at a much higher mass scale than FDM, so the amount of structure formed on such small mass scales in SFDMTF would be well below that in the corresponding, core-size-matched FDM model. On the other hand, if we align the $k_{\text {cut }}$-values of the two models, instead, which Fig. 6 tells us requires $R_{\mathrm{TF}} \lesssim 1$ pc, then much more small-scale power is retained below the cut-off in SFDM-TF than in FDM, as will be even clearer from the results for their power spectra and HMFs in the sections below. Hence, lower limits like those above on the particle masses for FDM, derived to keep FDM from underproducing structure on small scales, when used to infer upper limits on $R_{\mathrm{TF}}$ based upon $k_{\text {cut }}$-matching FDM, might then be overly restrictive in limiting $R_{\mathrm{TF}}$.

\subsubsection{Power spectra and RMS mass fluctuations}

In $\$ 4.7 .2$ above, we presented the normalized transfer function results for SFDM-TF and FDM, which are, by definition, expressed in units of the transfer function for CDM. In Fig. 7(a), we also present the power spectra $P(k)$ vs. $k$ for each model, separately, by multiplying those normalized transfer functions by the CDM transfer function generated by the publicly-available самв code. We present these power spectra, for several illustrative values of $R_{\mathrm{TF}}$ for SFDM-TF and $m_{22}$ for FDM, respectively, as labeled, linearly-extrapolated to $z=0$. This takes proper account of the effect of the smooth transition of our observed background Universe at late times, from MD to $\Lambda$-dominated (" $\Lambda \mathrm{D}$ ”), since all modes of interest to us have, by then, long-since transitioned to pressure-free CDM-like perturbation growth according to linear theory.

With these power spectra, we can also compute the RMS mass fluctuations $\sigma_{\mathrm{M}}(M)$ and their logarithmic derivatives, as plotted vs. $M$ in Figs. 7(b) and (c). The curves of $\sigma_{\mathrm{M}}(M)$ for SFDM-TF and FDM follow that for CDM at high mass, increasing towards smaller $M$, but they flatten to a contant value in the mass range associated with wavenumbers of modes which are suppressed by Jeans-filtering relative to the growth of CDM perturbations of the same wavenumber. From the plots of $\sigma_{\mathrm{M}}(M)$, alone, it is difficult to appreciate the differences between the SFDM-TF and FDM results, even when overplotting them so as to compare them directly, by choosing model parameters for SFDM-TF and FDM which give them similar values of $k_{\text {cut }}$. In particular, it is difficult to see from the curves in Fig. 7(b), the important distinction between the behaviors of the two models when switching between top-hat and sharp- $k$ filtering. The difference is more evident, however, when viewed by plotting the logarithmic derivatives of $\sigma_{\mathrm{M}}(M)$, instead, as shown in Fig. 7(c). For FDM, this logarithmic derivative at values of $M$ associated with wavenumbers above the cut-off in the transfer function approaches zero (from below) much more rapidly as $M$ decreases, for sharp- $k$ filtering than for top-hat filtering. We will see just how significant this difference is below, when we apply these results to calculate the HMF for FDM. For SFDM-TF, however, the logarithmic derivative shown in Fig. 7(c) for top-hat filtering captures the envelope of the rapidly-oscillating behavior when a sharp- $k$-filter is adopted, instead, so, when averaged over these oscillations, the results for the two filter functions are essentially the same.

\subsubsection{Halo mass functions}

In Fig. 8, we plot HMFs at $z=0$, computed from the RMS mass fluctuations and their logarithmic derivatives plotted in Fig. 7, for SFDM-TF (for $R_{\mathrm{TF}}=1000,100,10$ and $1 \mathrm{pc}$ ), FDM (for $m_{22}=0.8$ and 29), and CDM. As expected, all three models produce the same HMF at high mass, well above the scales affected by Jeans-filtering. Since Jeans-filtering of small-scale structure produces cut-offs at high wavenumber in the power spectra and transfer functions of both FDM and SFDM-TF, cut-offs appear in both their HMFs at small halo mass $M$, as well. However, as Fig. 8 makes clear, the shape and depth 

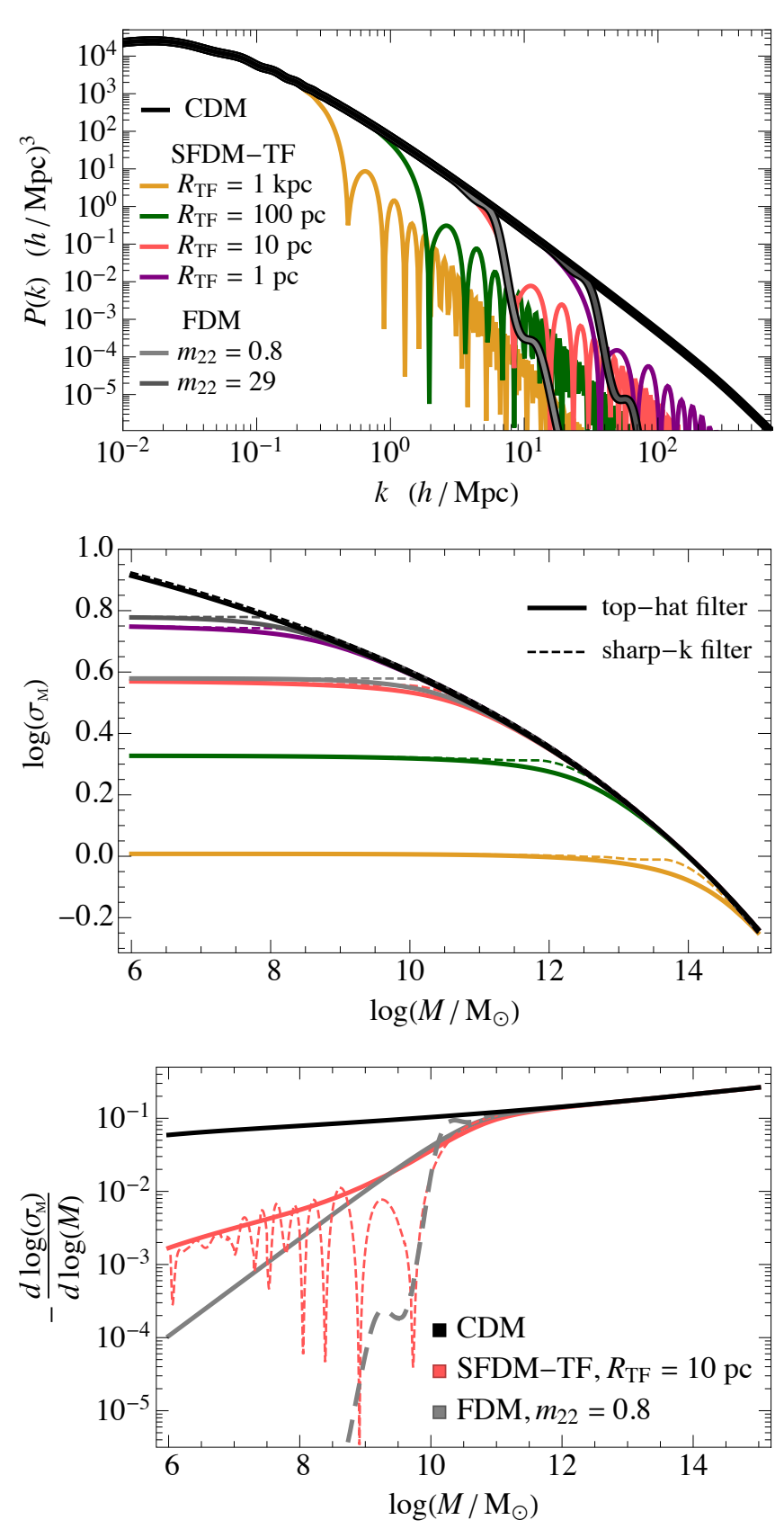

Figure 7. Power spectrum and RMS mass fluctuation at $z=0$. (a) (top panel) Present-day matter power spectra $P(k)$, (b) (middle panel) RMS mass fluctuation $\sigma_{\mathrm{M}}(M)$, and (c) (bottom panel) the logarithmic derivative of $\sigma_{\mathrm{M}}$, for CDM (black), FDM (grey), and SFDM-TF (colored) models, as labeled. The CDM and FDM curves are generated using AxionCAMB. The SFDM-TF curves are generated by multiplying the transfer functions of Fig. 6 (obtained through our linear perturbation calculation) by the CDM power spectrum from AXIONCAMB. In the middle and bottom panels, solid lines represent tophat filtering, while dashed lines represent sharp- $k$ filtering. The logarithmic derivative is much steeper for sharp- $k$ filtering than for top-hat filtering for FDM, while for SFDM-TF, top-hat filtering captures the envelope of the rapidly-oscillating sharp- $k$-filtered result, so they are essentially the same.
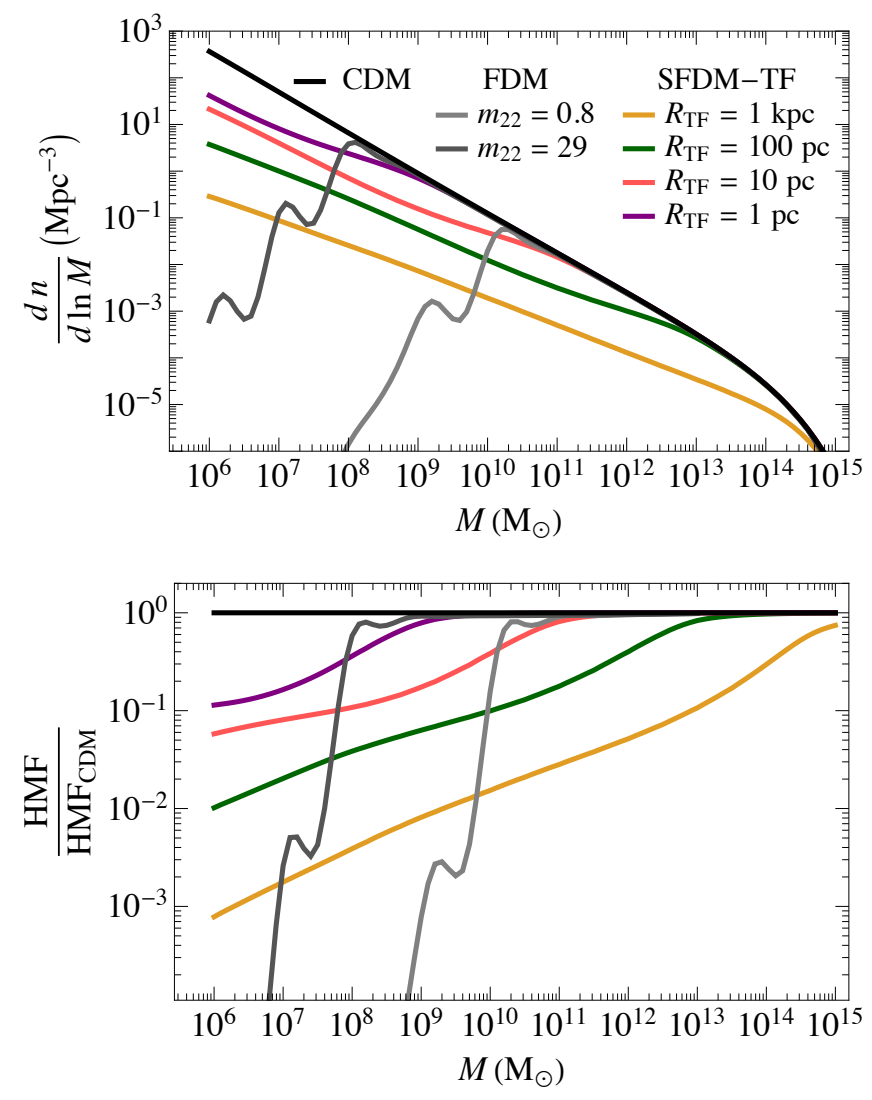

Figure 8. Halo mass function at $z=\mathbf{0}$. (a) (top panel) $\mathrm{HMFs}, d n / d \ln M$, are plotted vs. halo mass $M$ for SFDM-TF, CDM and FDM. SFDM-TF curves are for different $R_{\mathrm{TF}}$-values, $R_{\mathrm{TF}}=\{1000,100,10,1\}$ pc (colored curves from lowest to highest). CDM HMF (black curve) is computed from the transfer function generated by САмв. FDM HMFs (light and dark grey curves) are for different particle masses $m_{22}=\{0.8,29\}$, respectively, as computed from the transfer functions generated by Ахіолсамв. (b) (bottom panel) Same as (a), but normalized by the CDM HMF.

of this HMF cut-off for SFDM-TF are dramatically different from that for FDM. In particular, the cut-off for SFDM-TF is much weaker and more gradual than that for FDM. This will have strong consequences for the comparison between any observables for the two models that reflect a turn-down in their abundance of small-mass galactic haloes relative to that in the standard CDM model.

The origin of this difference can be understood from the curves for $\sigma_{\mathrm{M}}(M)$ and its logarithmic derivative for the two models, in Fig. 7(b) and (c). As we noted above, the sharp- $k$-filtered result for FDM is much more sharply cut-off at small mass than is the top-hat-filtered result. This is why sharp- $k$-filtered results are strongly preferred for this model (Kulkarni \& Ostriker 2020). This is generally true for other models with a similar cut-off of power on small scales, e.g. warm dark matter ("WDM"), as well. For this reason, we used the sharp- $k$-filtered results to compute the HMF for FDM in Fig. 8. For SFDM-TF, however, the choice of filter does not make a difference in how sharply $\sigma_{\mathrm{M}}(M)$ cuts off. Using the top-hat filter for SFDM-TF merely smooths over high-frequency oscillations in the logarithmic derivative of the sharp- $k$-filtered $\sigma_{\mathrm{M}}(M)$, but still traces their envelope very well, so the sharpness of the cut-off is the same for the two filters. Regardless of which filter is used, that cut-off is much more gradual and less severe for the SFDM-TF model than for FDM. 

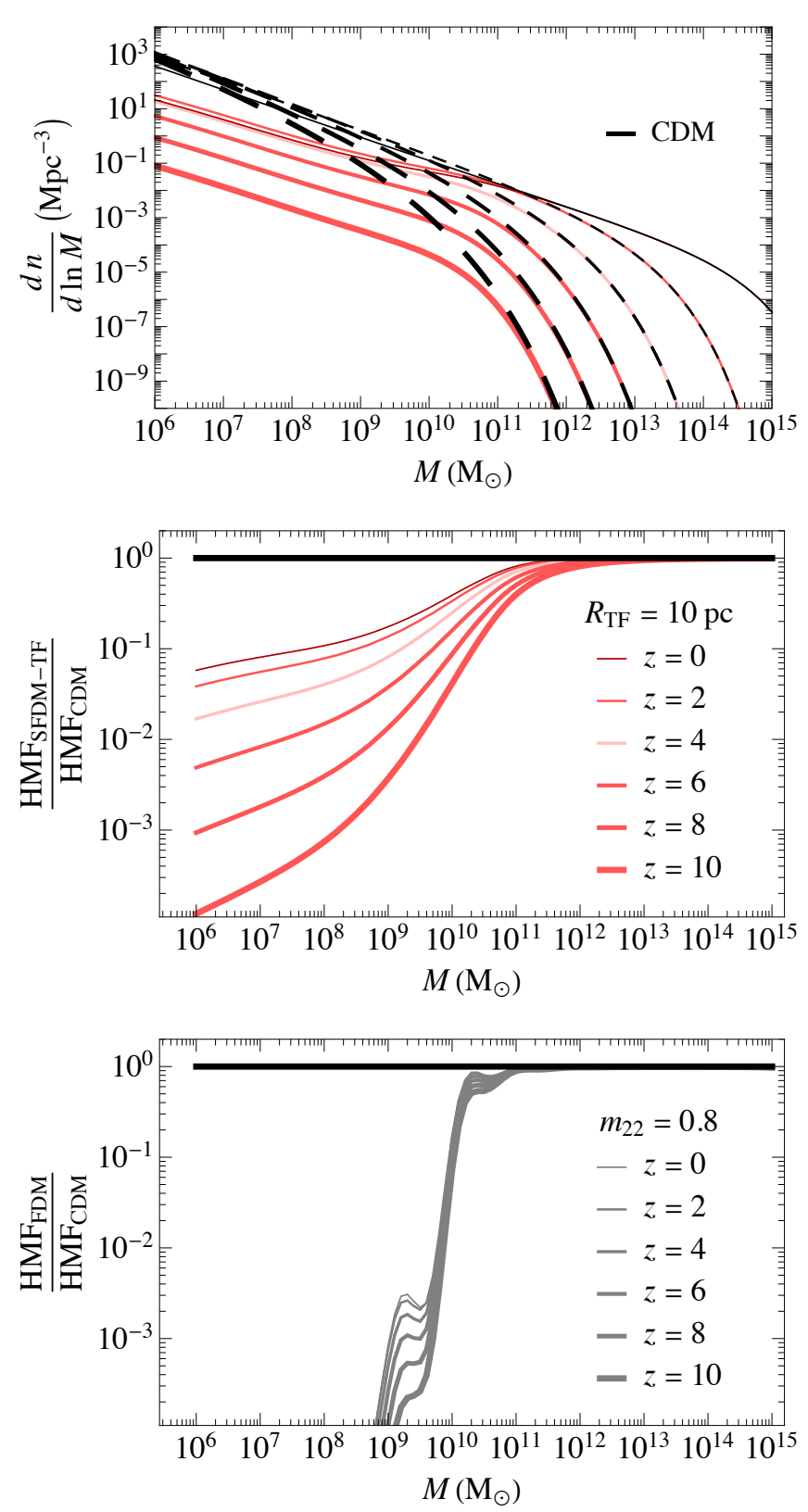

Figure 9. Evolution of the halo mass function. Same as Fig. 8, except for different redshifts as labeled, and the SFDM-TF curves are all for $R_{\mathrm{TF}}=10 \mathrm{pc}$, while the FDM curves are all for $m_{22}=0.8$. (a) (top panel) Same as Fig. 8(a) (but note the expanded $y$-axis scale), except for different redshifts and a single SFDM-TF case with $R_{\mathrm{TF}}=10 \mathrm{pc}$. CDM curves are dashed for readability. (b) (middle panel) Same as Fig. 8(b), except for different redshifts and a single SFDM-TF case with $R_{\mathrm{TF}}=10 \mathrm{pc}$. (c) (bottom panel) Same as (b), except for a FDM case with $m_{22}=0.8$.

We chose to plot the SFDM-TF HMF using the top-hat filter, rather than the sharp- $k$-filter, to avoid giving false prominence to the corresponding oscillations that appear in the HMF in this case, if the latter were used, instead. Their prominence and phases are artificially related to our thin-horizon and thin-EOS-transition approximations. However, our conclusion that the overall cut-off for the SFDM-TF case is much shallower and more gradual than for FDM is robust, as it is the same for both filters.
In view of the importance of this difference in the $z=0 \mathrm{HMFs}$ for SFDM-TF and FDM, we have explored the time-dependence of this difference in Fig. 9. By comparing the HMFs for the two models at redshifts ranging from $z=0$ to $z=10$, it is apparent that the smallmass cut-off for FDM, relative to that for CDM, is much sharper and much deeper than that for SFDM-TF, at all redshifts in this range. Moreover, there is a strong trend in the HMF for SFDM-TF at masses below the cut-off, for it to recover over time, gradually rising toward that for CDM. There is no comparable recovery for the more sharplyreduced HMF in the FDM case, sufficient to fill in the missing halo abundance at small mass relative to that of CDM.

For some purposes, when comparing predictions of "cut-off models" like these to CDM and to observations, the integrated HMFs over some low-mass halo ("LMH") mass range affected by the cut-off is also of interest. In Table 2, we show the integrated halo number and mass densities, given by

$n_{\mathrm{LMH}}=\int \frac{d n}{d \ln M} d \ln M=\int \frac{1}{M} \frac{d n}{d \ln M} d M$

and

$\rho_{\text {LMH }}=\int \frac{d n}{d \ln M} d M$

evaluated at $z=0$, for halo masses in the ranges $10^{6} \leq M / \mathrm{M}_{\odot} \leq$ $10^{11}$ and $10^{8} \leq M / \mathrm{M}_{\odot} \leq 10^{11}$, for two illustrative sets of pairings of model parameters for SFDM-TF vs. FDM, $R_{\mathrm{TF}}=10$ pc vs. $m_{22}=$ 0.8 , and $R_{\mathrm{TF}}=1$ pc vs. $m_{22}=29$, respectively, plotted in previous figures, including their results relative to CDM. When the minimum halo mass is $10^{8} \mathrm{M}_{\odot}$, SFDM-TF with $R_{\mathrm{TF}}=1 \mathrm{pc}$ has a halo number comparable to that of FDM with $m_{22}=29$, and within a factor of 2 of that for CDM, while the masses in all three models are very close. For $R_{\mathrm{TF}}=10 \mathrm{pc}$, however, SFDM-TF has many more haloes than does FDM with $m_{22}=0.8$, by an order of magnitude, and $14 \%$ of the CDM number, while the masses in both SFDM-TF and FDM are comparable and $\approx 1 / 3$ of that for CDM. If the minimum halo mass is lowered to $10^{6} \mathrm{M}_{\odot}$, however, SFDM-TF retains many more haloes than FDM for both example cases - by an order of magnitude for $R_{\mathrm{TF}}=1$ pc vs. $m_{22}=29$, and by more than a factor of 300 for $R_{\mathrm{TF}}=10$ pc vs. $m_{22}=0.8-$ and somewhat more halo mass in this low-mass range than the corresponding FDM models, despite the fact that the SFDM-TF cases have slightly smaller cut-off mass scales ( $M_{\text {cut }}$ ) than the corresponding FDM cases. In fact, the $R_{\mathrm{TF}}=10 \mathrm{pc}$ case even retains more haloes (by number) than the $m_{22}=29$ case. These examples make it clear that one must be careful when treating lower limits on $m_{22}$ for FDM as a proxy for providing upper limits on $R_{\mathrm{TF}}$ for SFDM-TF. If the former is derived to ensure that FDM has a high enough HMF at low mass, it may be overly restrictive if used to place an upper limit on the SFDM-TF $R_{\mathrm{TF}}$ simply by solving for the value that makes their cut-off wavenumbers the same.

This gradual cut-off of the HMF is a defining feature of SFDM-TF and distinguishes it from any other DM alternative with a cut-off in its power spectrum that reduces small-scale power relative to CDM as sharply as does FDM. Its origin is rooted in the "incredible shrinking Jeans mass" of SFDM-TF, which leads to a "recovery" of small-scale perturbations that were initially retarded below the cut-off when the Jeans scale was large, then free to grow like CDM after exceeding the Jeans scale, with plenty of time left to close the gap with CDM, at least partially. As a result, even haloes of mass well below $M_{\text {cut }}$ in the transfer function can still be present in significant numbers at late times.

In principle, this suggests that SFDM-TF might have an advantage over FDM, when it comes to resolving the problem CDM has of overpredicting dwarf satellites compared with observations of the 


\begin{tabular}{lcccc} 
& \multicolumn{2}{c}{$10^{6} \leq M / \mathrm{M}_{\odot} \leq 10^{11}$} & \multicolumn{2}{c}{$10^{8} \leq M / \mathrm{M}_{\odot} \leq 10^{11}$} \\
\hline \hline$R_{\mathrm{TF}}=10 \mathrm{pc}$ & $n_{\mathrm{LMH}}\left(\mathrm{Mpc}^{-3}\right)$ & $\rho_{\mathrm{LMH}}\left(10^{9} \mathrm{M}_{\odot} \mathrm{Mpc}^{-3}\right)$ & $n_{\mathrm{LMH}}\left(\mathrm{Mpc}^{-3}\right)$ & $\rho_{\mathrm{LMH}}\left(10^{9} \mathrm{M}_{\odot} \mathrm{Mpc}^{-3}\right)$ \\
$m_{22}=0.8$ & 29 & 3.2 & 1.1 & 3.0 \\
$R_{\mathrm{TF}}=1 \mathrm{pc}$ & 0.086 & 2.6 & 0.086 & 2.6 \\
$m_{22}=29$ & 61 & 7.1 & 4.2 & 6.7 \\
\hline & 7.6 & 7.0 & 6.0 & 6.9 \\
\hline$R_{\mathrm{TF}}=10 \mathrm{pc}$ & $n_{\mathrm{LMH}} / n_{\mathrm{LMH}, \mathrm{CDM}}$ & $\rho_{\mathrm{LMH}} / \rho_{\mathrm{LMH}, \mathrm{CDM}}$ & $n_{\mathrm{LMH}} / n_{\mathrm{LMH}, \mathrm{CDM}}$ & $\rho_{\mathrm{LMH}} / \rho_{\mathrm{LMH}, \mathrm{CDM}}$ \\
$m_{22}=0.8$ & 0.07 & 0.32 & 0.14 & 0.39 \\
$R_{\mathrm{TF}}=1 \mathrm{pc}$ & 0.0002 & 0.26 & 0.011 & 0.33 \\
$m_{22}=29$ & 0.14 & 0.71 & 0.55 & 0.87 \\
\hline
\end{tabular}

Table 2. Integrated halo number density and mass density of low-mass haloes ("LMH") in the range $10^{6} \leq M / \mathrm{M}_{\odot} \leq 10^{11}$ (left 2 columns) and $10^{8} \leq M / \mathrm{M}_{\odot} \leq 10^{11}$ (right 2 columns) at $z=0$ for SFDM-TF and FDM, including their ratios to the CDM quantities, for illustrative values of $R_{\mathrm{TF}}$ and $m_{22}$.

Milky Way and Local Group. In particular, if FDM uses its cut-off to suppress the abundance of dwarf satellites so as to reconcile with observations at the higher-mass end (i.e. at the scale of the so-called "too big to fail" dwarfs - henceforth, "TBTF"), it tends to eliminate lower-mass haloes (e.g. the ultra-faint dwarfs) too drastically. Since the more gradual cut-off in the HMF of SFDM-TF might avoid this difficulty, we consider this further, as follows.

Predicted dwarf satellite abundances can be expressed in terms either of the number of haloes per interval of halo mass or their number per interval of halo circular velocity (as measured at some radius), which decline with increasing halo mass and velocity, respectively. The observational problem for CDM is usually expressed in terms of the latter, more observable property, involving velocity, since it can be determined from a rotation curve, observed only part-way out in total mass. There are, in principle, two ways to modify the predicted velocity distribution function in the TBTF mass range so as to reduce the overabundance in CDM N-body simulations to make them agree with the observed distribution:

(1) reduce the halo number per unit mass without altering their internal densities relative to CDM haloes, or

(2) reduce the internal densities of the haloes without changing their number, so haloes of a given mass appear at lower velocity but match the lower abundance of CDM haloes of higher velocity.

(TBTF haloes are so named because they are too massive to be made undetectable by suppressing their ability to form stars, which would otherwise be a third option for avoiding their apparent overabundance in CDM.)

FDM has trouble with both options, as follows. If $m_{22} \approx 1$, TBTF haloes (e.g. with $M \approx 10^{10} \mathrm{M}_{\odot}$ ) have their internal densities lowered by the presence of solitonic cores as large as their de Broglie wavelengths of a few kpc or more. However, while this helps to solve the TBTF problem by option (2), it also reduces the abundance in the HMF, including a catastrophic reduction at lower-mass where ultra-faint dwarfs are detected. If, on the other hand, FDM tries to avoid the latter problem by choosing a large enough $m_{22}, m_{22} \geq 29$ (Nadler et al. 2021), then neither option (1) nor (2) are available to solve the TBTF problem. While the value of $m_{22}=29$ is just large enough to make sure the HMF in the range of ultrafaint dwarfs is not over-reduced, the cut-off is so sharp that the number of haloes is then not significantly reduced in the TBTF mass range, either, so option (1) fails. And, unfortunately, the solitonic cores of the TBTF mass range are smaller in that case, since their de Broglie wavelengths are then $\lesssim 0.1 \mathrm{kpc}$, which is too small to flatten the central density profiles enough to lower the rotation-curve velocities to make option (2) possible. Such small cores are also too small for FDM to solve the cusp-core problem of CDM. For that problem, it turns out, even the smaller value of $m_{22} \approx 1$ is not a good solution, since the solitonic cores of galaxies even more massive than TBTF galaxies (e.g. $M \gtrsim 10^{11} \mathrm{M}_{\odot}$ ) are then too dense, even denser than in CDM (Robles et al. 2019).

For SFDM-TF, on the other hand, option (2) requires $R_{\mathrm{TF}} \gtrsim 1 \mathrm{kpc}$, in order to make large enough polytropic cores to lower the densities of TBTF haloes sufficiently, which would also help to resolve the cusp-core problem (Paper I). However, for halo formation from cosmological initial conditions, our results in Fig. 8 show that, if $R_{\mathrm{TF}}$ is this large, the cut-off mass in the HMF is so large that halo abundances at all galactic halo masses are substantially reduced relative to CDM. Hence, even though it is a much more gradual cut-off than FDM's, it still over-reduces the dwarf satellite abundance relative to CDM. So, option (2) is not available to SFDM-TF, either. According to the results in Table 2, in fact, if $m_{22} \geq 29$ is required for FDM to avoid under-predicting the observed abundance of ultrafaint dwarfs, then SFDM-TF probably requires $R_{\mathrm{TF}} \lesssim 1 \mathrm{pc}$. According to the HMF in Fig. 8 for $R_{\mathrm{TF}}=1 \mathrm{pc}$, however, the halo abundance at the TBTF mass scale is then just the same as for CDM, so option (1) is unavailable just as it was for FDM with $m_{22}=29$. This is true despite the fact that the FDM HMF cut-off just below the assumed ultrafaint dwarf mass range of $\gtrsim 10^{8} \mathrm{M}_{\odot}$ is much sharper than that for SFDM-TF with $R_{\mathrm{TF}}=1 \mathrm{pc}$. That property does give SFDM-TF one advantage over FDM, however, since it enables SFDM-TF to contribute a population of even smaller-mass subhalos which may help to account for perturbers of stellar streams observed in the Milky Way, which FDM may not (e.g. Banik et al. 2021).

While this may suggest that SFDM-TF is not much better at resolving the TBTF problem than FDM, in the face of the recently tightened constraint on the latter imposed by Nadler et al. (2021) based on the abundance of ultrafaint dwarfs in the Milky Way and Local Group, there are caveats to this conclusion. Firstly, our discussion is so far based only upon the comparison of the global HMFs of SFDM-TF, FDM and CDM, while dwarf satellites are subhalos within these larger halos. A conditional HMF which predicts the abundances of subhalos of a given mass within a halo of a larger mass at a given epoch might make the comparison between FDM and SFDM-TF different from that based upon comparing their global HMFs. Sec- 
ondly, if the masses of ultrafaint dwarfs, which are uncertain, are determined to be below $10^{8} \mathrm{M}_{\odot}$, then the more gradual cut-off of SFDM-TF gives it a much better chance of explaining TBTF than FDM, by allowing a larger value of $R_{\mathrm{TF}}$ (e.g. $R_{\mathrm{TF}} \gtrsim 10 \mathrm{pc}$ ) without then fatally underproducing the ultrafaint dwarfs. This is apparent if we compare the results for the two halo mass ranges in Table 2: in the range $10^{6} \leq M / \mathrm{M}_{\odot} \leq 10^{11}$, the $R_{\mathrm{TF}}=10 \mathrm{pc} \mathrm{SFDM-TF}$ model contains more haloes than the $m_{22}=29$ FDM limit, while this is not true in the range $10^{8} \leq M / \mathrm{M}_{\odot} \leq 10^{11}$. Thirdly, comparisons of the observations with these predicted HMFs also says nothing about the impact of dynamical processes inside the parent halo which can alter the subhalo HMFs there over time, e.g. by tidal disruption, which may also be different for SFDM-TF and FDM. Ultimately, 3D-cosmological simulations with high resolution and dynamic range, from initial conditions that led to the formation of the Local Group and its surroundings, may be required to settle the matter.

For values of $R_{\mathrm{TF}} \ll 1$ pc or $m_{22} \gg 29$, the HMFs and internal halo structure of both models approach that of CDM, for most observables. In that case, the same solutions to the small-scale structure problems of CDM that depend upon modifications of halo structure and abundance at the low-mass end by baryonic effects, are also available to solve the same problems for SFDM-TF or FDM. However, it may still be possible to distinguish the three models at even smaller halo mass, e.g. in the minihalo mass range, below $\approx 10^{8} \mathrm{M}_{\odot}$. According to the results for $z=0$ in Table 2, the more gradual cut-off of the HMF for SFDM-TF, relative to FDM, means that, for $R_{\mathrm{TF}}=1$ pc, there is still a significant population of minihalos for SFDM-TF, albeit several times reduced from that for CDM, but an order of magnitude more than for FDM with $m_{22}=29$.

\section{SUMMARY AND CONCLUSIONS}

In this paper and its companion Paper I, we considered the gravitational dynamics and quantum hydrodynamics of an alternative form of cosmic dark matter to standard CDM - SFDM-TF - a scalar field comprised of ultralight bosons with a repulsive self-interaction (SI) strong enough to make the length scale, which characterizes that SI, larger than the de Broglie wavelength (i.e. the Thomas-Fermi (TF) regime). Here, in Paper II, we studied structure formation in a universe with this kind of dark matter in a cosmological context, including the dynamics of both nonlinear collapse leading to halo formation and the linear perturbations which form the initial conditions for structure formation in this model. For the former, we applied the tools developed in Paper I to simulate cosmological infall and collapse to form individual virialized haloes. As the first step in understanding how cosmological initial and boundary conditions affect the outcome, we replaced the non-cosmological initial conditions of Paper I with CDM-like linear initial conditions. The latter were derived in order to make spherical infall occur at the rate which matches the empirical mass-accretion history (MAH) of haloes derived from N-body simulations of CDM. Our previous application of this method for fixing the initial perturbation for CDM simulations showed that CDM haloes formed in this way have NFW-like mass and phase-space density profiles, including the time-dependence of the NFW parameters of an individual halo as it grows, in close agreement with CDM N-body simulations (Alvarez et al. 2003; Shapiro et al. 2004, 2006). So, this provided an excellent test-bed for determining the impact on this problem of replacing CDM with SFDM-TF in the cosmological context of the expanding universe.

The SFDM-TF haloes that resulted (see §3.3) shared some prop- erties of those in Paper I, namely, polytropic cores within radius $R_{\mathrm{TF}}$ and outer envelopes that matched those of a simulation with CDM dynamics from the same initial conditions, although in this case, the outer-envelope profiles were NFW-like, as expected. In Paper I, the envelopes were power-law profiles, $\rho \propto r^{-12 / 7}$, everywhere outside the cores, just as for CDM simulations from the same non-cosmological (i.e. static) initial conditions. In the cosmological case here, however, between the core and the NFW-like outer envelope was an intermediate zone with the same power-law profile, $\rho \propto r^{-12 / 7}$, as Paper I found for its entire envelope outside the core. The result of Paper I that haloes form with polytropic cores surrounded by CDM-like envelopes is still an accurate description of the cosmological case, too, because the infall and collapse that leads to accretion shocks and virialized regions inside the shock are essentially CDM-like dynamics outside the polytropic cores in both cases. In other words, the term CDM-like characterizes the structure outside the cores in both cases, just from different initial conditions. The reason the cosmological SFDM-TF outcome exhibits both kinds of CDM-like envelope structure in the same halo - a power-law like the CDM simulation from non-cosmological initial conditions, surrounded by the NFW-like profile of CDM simulated in the cosmological case - is explained by the early smoothing effect that SI has on those initial conditions while they are still linear. An SI-pressuredriven sound wave was found to smooth-out the mass within a proper radius $R_{\mathrm{TF}}$ of the center, in a time short compared to the time for that mass to expand cosmologically, turn around and recollapse. Once smoothed, that mass continues expanding, but thereafter evolves like a top-hat perturbation, at the smoothed-out overdensity. When this mass collapses, the outcome for it is just like that in Paper I, while the mass outside it evolves just as it would for those CDM-like initial conditions with no smoothing effect.

This has profound consequences for halo formation from cosmological perturbations in SFDM-TF. If SI is too strong, i.e. $R_{\mathrm{TF}}$ is too large, the SI-smoothing sound wave can smooth-out a mass as large as that of the halo destined to form at some epoch in CDM from the same initial conditions, preventing its formation, altogether, or at least delaying it significantly. In Paper I, we showed how SFDM-TF haloes with the right value of $R_{\mathrm{TF}}$ could resolve the cusp-core and TBTF problems of CDM, without suffering from the problem identified for FDM by Robles et al. (2019) in which the solitonic cores of larger-mass FDM haloes were too dense to be consistent with the rotation curves of more massive galaxies, if FDM particle mass were tuned to $m_{22} \simeq 1$ to solve the other problems. We found that $R_{\mathrm{TF}} \gtrsim 1 \mathrm{kpc}$ is required. It was left to Paper II, here, to determine if such a value of $R_{\mathrm{TF}}$ was consistent with the cosmological formation of those haloes. Our cosmological simulations here indicate that the SI-smoothed mass at early times is large enough to suppress the formation of such dwarf galaxies if $R_{\mathrm{TF}} \gtrsim 1 \mathrm{kpc}$, if we start from CDM initial conditions. At the other extreme, if $R_{\mathrm{TF}} \lesssim 1 \mathrm{pc}$, then the internal structure of haloes that form from such CDM-like initial conditions would closely resemble those of CDM, over most of the observable range of radii.

This still left open the question of what realistic cosmological initial conditions are for SFDM-TF halo formation, as distinct from those for CDM. To answer that requires linear perturbation theory, from which the statistical likelihood of finding haloes of different masses at different epochs, can be determined. That was the subject of $\S 4$. There, we derived the transfer function for SFDM-TF, normalized by that for CDM, and, for comparison, following the same procedure, did the same for FDM, to contrast the three models. With the transfer function, we were able to calculate statistical measures of structure formation for the SFDM-TF model, for the first time, 
including its halo mass function (HMF). Just as the FDM model is fully parameterized by its particle mass $m$, so SFDM-TF is parameterized entirely by its SI strength parameter, $\mathrm{g} / \mathrm{m}^{2}$, which is directly related to the radius $R_{\mathrm{TF}}$ of the ( $\left.n=1\right)$-polytropic sphere in hydrostatic equilibrium in which gravity is balanced by SI pressure. Like FDM, SFDM-TF is a model with a cut-off of power on small scales, reflected as a cut-off at high wavenumber in the transfer function. As a result, we used these findings to make a first cut at estimating the range of $R_{\mathrm{TF}}$-values which are consistent with astronomical observations, by using constraints on the FDM model derived elsewhere, expressed as constraints on its $m_{22}$, as a proxy.

According to our linear perturbation theory presented in $\S 4$, the Fourier mode at which SFDM-TF cuts off relative to CDM has a comoving mass associated with it that equalled the time-varying Jeans mass at the moment that mode entered the horizon (i.e. filled the Hubble volume). With a Jeans length equal to $R_{\mathrm{TF}}$, fixed in proper coordinates, the comoving Jeans mass during this phase decreased rapidly with scale factor, as $M_{\mathrm{J}, 0} \propto a^{-3}$. Modes of smaller wavenumber entered the horizon later, with larger associated masses, above the Jeans mass, so they always grew like CDM. Modes of higher wavenumber entered earlier, however, when their associated masses were below the Jeans mass and, so, were initially retarded relative to $\mathrm{CDM}$, but, as soon as that rapidly shrinking Jeans mass reached their mass scale, they, too, were free, thereafter, to grow like CDM. The cosmological story of structure formation in SFDM-TF is, therefore, very much a tale of "the incredible shrinking Jeans mass", which is the key to understanding how it differs from other cut-off models like FDM (for which the Jeans mass only drops weakly, as $M_{\mathrm{J}, \mathrm{F}} \propto a^{-3 / 4}$ ) which do not share this unique property. Another factor that distinguishes SFDM-TF from FDM, in particular, is that, even when SFDM-TF modes are sub-Jeans, they still grow, albeit modestly, as $a^{1 / 4}$ (when the field is matter-like), while for FDM, there is no sub-Jeans growth.

This explains why, when we applied the same treatment to FDM to identify its cut-off wavenumber (as a function of $m_{22}$ ), and solved for the $R_{\mathrm{TF}}$-values required to align the cut-off wavenumbers of the two models, SFDM-TF haloes had SI-polytropic halo cores (of radius $R_{\mathrm{TF}}$ ) much smaller than the solitonic cores of FDM haloes of the same mass. For example, FDM with $m_{22} \simeq 1$, for which a $10^{10} \mathrm{M}_{\odot}$ halo has a solitonic core size (of order the de Broglie wavelength) of $\simeq 4 \mathrm{kpc}$, cuts off at close to the same wavenumber as SFDM-TF with $R_{\mathrm{TF}} \simeq 3 \mathrm{pc}$, which gives a halo of that mass a polytropic core $\approx 1000$ times smaller. This is consistent with our simulations in $\S 3.3$, which showed that halo formation in SFDM-TF from a CDM-like perturbation experiences early SI-smoothing that, in effect, "Jeansfilters" a mass closer to the initial value of the Jeans mass than to its smaller value after the collapse is finished. As a result, although SFDM-TF haloes with flattened cores as large as $R_{\mathrm{TF}} \gtrsim 1 \mathrm{kpc}$, formed by Jeans instability and collapse from a static initial condition, were shown in Paper I to be capable of resolving the cusp-core problem of CDM while simultaneously solving its TBTF problem, as well, this unified explanation is disfavored, once formation is placed in the context of cosmological perturbation growth.

Observational constraints on FDM can be used as a proxy for those on SFDM-TF by solving for the matching $m_{22}$ and $R_{\mathrm{TF}}$ values that make their respective transfer functions cut off at the same wavenumber, e.g. as estimated analytically by equation (71). Care should be taken to avoid FDM constraints that are based upon phenomenology that might distinguish it from SFDM-TF because of their different dynamics. Since lowering $m_{22}$ increases the de Broglie wavelength and extends the mass range suppressed by FDM to larger scales, many of its observational constraints are expressed as lower limits on $m_{22}$ required to avoid over-suppressing structure. There is a long list of attempts to estimate or limit $m_{22}$, with results roughly spanning the range from 1 to 30 (as summarized, e.g., in Grin et al. 2019). Some are based upon the comparison of the abundance of haloes or subhaloes with the predicted HMF for FDM. Nadler et al. (2021), for example, compare the predicted HMF of subhaloes to the observed dwarf galaxies in the Local Group and find that $m_{22} \gtrsim 29$ is required, corresponding to $\lambda_{\mathrm{deB}} \lesssim 0.5 \mathrm{kpc}$. Others are based upon more complicated, nonlinear dynamical phenomena. For example, the requirements of not dynamically over-heating the Milky Way disk or its stellar streams were used to place bounds of $m_{22} \gtrsim 0.6-1.5$ (Amorisco \& Loeb 2018; Church et al. 2019). Still others are based upon structure on larger scales, at epochs when it is only quasi-linear, but where one must model effects on the baryonic component, too, such as fluctuations in the matter-density field inferred from the power spectrum of measured fluctuations in the Lyman- $\alpha$ forest. Bounds inferred from the Lyman- $\alpha$ forest were the first to pose the "catch-22 problem" for FDM, in fact, finding $m_{22} \gtrsim 20-30$ (Armengaud et al. 2017; Iršič et al. 2017), now consistent with the recent lower limits quoted above from the abundance of dwarf satellites in the Local Group.

If the observational constraints on FDM we use as our proxy for those on SFDM-TF are taken to be $1 \lesssim m_{22} \lesssim 30$, then the range of $R_{\mathrm{TF}}$-values for which the two models have the same cut-off wavenumber corresponds roughly to $3 \mathrm{pc} \gtrsim R_{\mathrm{TF}} \gtrsim 0.2 \mathrm{pc}$. Of all the methods for constraining the FDM model we might use, as a more specific proxy than this to constrain SFDM-TF, those comparing their HMFs are, to first approximation, less affected by additional differences in their dynamics than others. For this reason, we focused most of our additional comparison here on the results of their HMF predictions, as discussed in $\$ 4.7 .4$. We showed there that, for the same cut-off wavenumbers, the SFDM-TF HMF is much more gradually cut off than that of FDM, so it retains many more haloes at the lowmass end. As a result, if we were, instead, to solve for the $R_{\mathrm{TF}}$-value that yields the same halo number as FDM for some value of $m_{22}$, the corresponding $R_{\mathrm{TF}}$-value is generally larger than the one that makes the cut-off wavenumbers match, described above. For example, if we integrate over their HMFs to count haloes above $10^{8} \mathrm{M}_{\odot}$, SFDM-TF with $R_{\mathrm{TF}}=1 \mathrm{pc}$ has a halo number comparable to that of FDM with $m_{22}=29$, and within a factor of 2 of that for CDM, while the integrated collapsed fractions in this halo mass range in all three models are very close. In that case, we should multiply the $R_{\mathrm{TF}^{-}}$ value above that matches the cut-off wavenumber for this $m_{22}$ by a factor of 5 , from 0.2 to $1 \mathrm{pc}$, to use the HMF FDM constraint as a proxy for SFDM-TF. Moreover, if $R_{\mathrm{TF}}=10 \mathrm{pc}$, SFDM-TF has many more haloes than does FDM with $m_{22}=0.8$, by an order of magnitude, and still has $14 \%$ of the CDM number. In that case, we should refine the first proxy range estimated above, upward, at least to $10 \mathrm{pc} \gtrsim R_{\mathrm{TF}} \gtrsim 1 \mathrm{pc}$. If we compare the integrated halo counts in the two models down to even smaller halo mass, the corresponding $R_{\mathrm{TF}}$-values shift upward even further. If the minimum halo mass is lowered to $10^{6} \mathrm{M}_{\odot}$, for example, then SFDM-TF with $R_{\mathrm{TF}}=1 \mathrm{pc}$ has an order of magnitude more haloes than does FDM with $m_{22}=29$, while if $R_{\mathrm{TF}}=10 \mathrm{pc}$, it has more by a factor of 300 than FDM with $m_{22}=0.8$. This indicates that one must be careful not to take the simple alignment of the cut-off wavenumbers of SFDM-TF and FDM as a basis for using the observational constraints on $m_{22}$ to solve for the corresponding constraints on $R_{\mathrm{TF}}$ - that will generally be overly restrictive, with upper limits on $R_{\mathrm{TF}}$ which are too low. It further suggests that SFDM-TF may have some advantages over FDM since, even when their model parameters are tuned so as to match HMFs at larger mass scales, SFDM-TF may still have more smaller-mass 
haloes. For example, when FDM and SFDM-TF are both limited so as to avoid over-suppressing the dwarfs in the Local Group, SFDMTF may still have enough even-smaller-mass subhalos to account for the perturbers of stellar streams observed in the Milky Way, even if FDM does not (e.g. Banik et al. 2021).

To test SFDM-TF against observations more directly in future work, it will be necessary to revisit the full list of phenomenological constraints that have been applied to FDM and other models that suppress small-scale structure as a built-in feature (e.g. warm dark matter), with SFDM-TF initial conditions and dynamics, instead. This will require us to apply the cosmological initial conditions derived here for SFDM-TF in fully 3D simulations of galaxy and large-scale structure formation, which are under development. This includes the coupling of dark matter and baryonic components, as well as pure dark matter simulation. For "cut-off models" like these, the nature of structure formation on scales at or below the cut-off is different for different types of dark matter, even when they start from transfer functions that have similar cut-off scales, as our results here demonstrate by comparing the HMFs for SFDM-TF and FDM. Additional effects, like fragmentation of pancakes and filaments, for example, can also introduce novel small-scale structures, unfamiliar from the hierarchical clustering paradigm of CDM (e.g. Mocz et al. 2019; Valinia et al. 1997). The small- $R_{\mathrm{TF}}$-regime favored thus far by our results presented above, for which the polytropic core sizes are subgalactic (i.e. sub-kpc), may present other novel features which are detectable, as well, worth exploring further. As discussed in Padilla et al. (2021), for example, such cores, if dense enough, may be subject to general relativistic instability leading to collapse to form supermassive black holes or their seeds.

In this paper, we considered the rôle of SI in structure formation in the SFDM model, in the TF regime. We also compared the results in this regime with the other limiting case, of FDM, in which SI is absent. As we showed in $\S 4.3$, as long as the particle mass and interaction strength place the scalar field in this regime at late times (e.g. the present), it is in the TF regime at earlier times, as well. In the future, we will consider the possibility of a transitional regime between these two limits.

\section{ACKNOWLEDGEMENTS}

This material is based upon work supported by the National Science Foundation Graduate Research Fellowship Program under Grant No. DGE-1610403. Any opinions, findings, and conclusions or recommendations expressed in this material are those of the authors and do not necessarily reflect the views of the National Science Foundation. T.R.-D. is supported by the Austrian Science Fund FWF through an Elise Richter fellowship, Grant No. V 656-N28. Simulations presented here were conducted on the Texas Advanced Computing Center's Stampede2 supercomputer under accounts asoz-630 and NSF XSEDE account TG-AST090005. The authors gratefully acknowledge The Incredible Shrinking Man (1957) [Film], directed by Jack Arnold, Universal-International Pictures Co., Inc., as the inspiration for our subtitle.

\section{DATA AVAILABILITY STATEMENT}

The data underlying this article are available in the article and in its online supplementary material.

\section{REFERENCES}

Ahn K., Shapiro P. R., 2005, MNRAS, 363, 1092

Alvarez M. A., Ahn K., Shapiro P. R., 2003, in Reyes-Ruiz M., VázquezSemadeni E., eds, Revista Mexicana de Astronomia y Astrofisica Conference Series Vol. 18, Revista Mexicana de Astronomia y Astrofisica Conference Series. pp 4-7 (arXiv: astro-ph/0302336)

Amorisco N. C., Loeb A., 2018, preprint, arXiv:1808.00464

Armengaud E., Palanque-Delabrouille N., Yèche C., Marsh D. J. E., Baur J., 2017, MNRAS, 471, 4606

Balberg S., Shapiro S. L., Inagaki S., 2002, ApJ, 568, 475

Banik N., Bovy J., Bertone G., Erkal D., de Boer T. J. L., 2021, MNRAS, 502,2364

Bardeen J. M., Bond J. R., Kaiser N., Szalay A. S., 1986, ApJ, 304, 15

Benson A. J., et al., 2013, MNRAS, 428, 1774

Bond J. R., Cole S., Efstathiou G., Kaiser N., 1991, ApJ, 379, 440

Bullock J. S., Boylan-Kolchin M., 2017, ARA\&A, 55, 343

Chavanis P. H., 2012, A\&A, 537, A127

Church B. V., Mocz P., Ostriker J. P., 2019, MNRAS, 485, 2861

Dawoodbhoy T., Shapiro P. R., Rindler-Daller T., 2021, MNRAS, 506, 2418 (Paper I)

Desjacques V., Kehagias A., Riotto A., 2018, Phys. Rev. D, 97, 023529

Diemer B., 2018, ApJS, 239, 35

Goodman J., 2000, New Astron., 5, 103

Grin D., Amin M. A., Gluscevic V., Hlozek R., Marsh D. J. E., Poulin V., Prescod-Weinstein C., Smith T., 2019, BAAS, 51, 567

Guzmán F. S., Ureña-López L. A., 2004, Phys. Rev. D, 69, 124033

Hlozek R., Grin D., Marsh D. J. E., Ferreira P. G., 2015, Phys. Rev. D, 91 , 103512

Hoffman Y., Shaham J., 1985, ApJ, 297, 16

Hu W., Barkana R., Gruzinov A., 2000, Phys. Rev. Lett., 85, 1158

Iršič V., Viel M., Haehnelt M. G., Bolton J. S., Becker G. D., 2017, Phys. Rev. Lett., 119, 031302

Khlopov M. I., Malomed B. A., Zeldovich I. B., 1985, MNRAS, 215, 575

Klypin A., Yepes G., Gottlöber S., Prada F., Heß S., 2016, MNRAS, 457, 4340

Knebe A., et al., 2013, MNRAS, 435, 1618

Kulkarni M., Ostriker J. P., 2020, preprint, arXiv:2011.02116

Lee J.-W., Koh I.-G., 1996, Phys. Rev. D, 53, 2236

Li B., Rindler-Daller T., Shapiro P. R., 2014, Phys. Rev. D, 89, 083536

Li B., Shapiro P. R., Rindler-Daller T., 2017, Phys. Rev. D, 96, 063505

Marsh D. J. E., 2016, Phys. Rep., 643, 1

Matos T., Guzmán F. S., Ureña-López L. A., 2000, Classical and Quantum Gravity, 17, 1707

Matos T., Vázquez-González A., Magaña J., 2009, MNRAS, 393, 1359

Mo H., van den Bosch F., White S., 2010, Galaxy Formation and Evolution. Cambridge University Press, doi:10.1017/CBO9780511807244

Mocz P., Vogelsberger M., Robles V. H., Zavala J., Boylan-Kolchin M., Fialkov A., Hernquist L., 2017, MNRAS, 471, 4559

Mocz P., Lancaster L., Fialkov A., Becerra F., Chavanis P.-H., 2018, Phys. Rev. D, 97, 083519

Mocz P., et al., 2019, Phys. Rev. Lett., 123, 141301

Mukhanov V., 2005, Physical Foundations of Cosmology. Cambridge University Press, doi:10.2277/0521563984

Nadler E. O., et al., 2021, Phys. Rev. Lett., 126, 091101

Navarro J. F., Frenk C. S., White S. D. M., 1997, ApJ, 490, 493

Padilla L. E., Rindler-Daller T., Shapiro P. R., Matos T., Vázquez J. A., 2021, Phys. Rev. D, 103, 063012

Peebles P. J. E., 2000, ApJ, 534, L127

Penston M. V., 1969, MNRAS, 144, 425

Planck Collaboration et al., 2020, A\&A, 641, A6

Press W. H., Schechter P., 1974, ApJ, 187, 425

Rindler-Daller T., Shapiro P. R., 2012, MNRAS, 422, 135

Rindler-Daller T., Shapiro P. R., 2014, Modern Physics Letters A, 29, 1430002

Robles V. H., Bullock J. S., Boylan-Kolchin M., 2019, MNRAS, 483, 289

Schive H.-Y., Chiueh T., Broadhurst T., 2014, Nature Physics, 10, 496

Schumann M., 2019, Journal of Physics G Nuclear Physics, 46, 103003

Schwabe B., Niemeyer J. C., Engels J. F., 2016, Phys. Rev. D, 94, 043513 
Shapiro P. R., Iliev I. T., Martel H., Ahn K., Alvarez M. A., 2004, preprint, arXiv:astro-ph/0409173

Shapiro P. R., Ahn K., Alvarez M., Iliev I. T., Martel H., 2006, in Mamon G. A., Combes F., Deffayet C., Fort B., eds, EAS Publications Series Vol. 20, EAS Publications Series. pp 5-10 (arXiv:astro-ph/0510146), doi:10.1051/eas:2006036

Sin S.-J., 1994, Phys. Rev. D, 50, 3650

Skodje R. T., Rohrs H. W., Vanbuskirk J., 1989, Phys. Rev. A, 40, 2894

Suárez A., Chavanis P.-H., 2015, Phys. Rev. D, 92, 023510

Suárez A., Chavanis P.-H., 2017, Phys. Rev. D, 95, 063515

Suárez A., Matos T., 2011, MNRAS, 416, 87

Suárez A., Robles V. H., Matos T., 2014, in Moreno González C., Madriz Aguilar J. E., Reyes Barrera L. M., eds, Accelerated Cosmic Expansion Vol. 38, Accelerated Cosmic Expansion. p. 107 (arXiv: 1302.0903), doi:10.1007/978-3-319-02063-1_9

Valinia A., Shapiro P. R., Martel H., Vishniac E. T., 1997, ApJ, 479, 46

Wechsler R. H., Bullock J. S., Primack J. R., Kravtsov A. V., Dekel A., 2002, ApJ, 568, 52

Widrow L. M., Kaiser N., 1993, ApJ, 416, L71

\section{APPENDIX A: OVERDENSITY PROFILE FOR NON-SELF-SIMILAR SPHERICAL INFALL: NFW-PRODUCING INITIAL CONDITIONS}

In order to obtain an exact expression for equations (37) and (38), we calculate the initial mean overdensity of Lagrangian mass shells that accrete onto the halo (i.e. reach close to $R_{200}$ ) at times that are consistent with the MAH of equation (36). Prior to the scale factor at which each shell has accreted, $a_{200}(M)$, the pressure forces are negligible, so the shell's trajectory is well-described by pressurefree, spherical top-hat collapse, governed by Newtonian gravitational motion:

$$
\begin{aligned}
\frac{d^{2} r}{d t^{2}} & =-\frac{G M}{r^{2}} \\
& =-\frac{2}{9} \frac{r}{t^{2}}(1+\Delta)
\end{aligned}
$$

where the last expression comes from the definition of the mean overdensity of a shell embedded in an EdS background universe for which $\rho_{\text {crit }}=\left(6 \pi G t^{2}\right)^{-1}$,

$1+\Delta=\frac{M}{\frac{4 \pi}{3} r^{3} \rho_{\text {crit }}}=\frac{9}{2} \frac{G M t^{2}}{r^{3}}$

Equation (A2) yields parametric solutions for each $M$ (see, e.g., Mo et al. 2010, §5.1.1):

$$
\begin{aligned}
r(M, a) & =\frac{1}{2} \frac{r\left(M, a_{i}\right)}{5 \Delta_{\mathrm{L}}\left(M, a_{i}\right) / 3}(1-\cos \theta) \\
t(a) & =\frac{3}{4} \frac{t\left(a_{i}\right)}{\left[5 \Delta_{\mathrm{L}}\left(M, a_{i}\right) / 3\right]^{3 / 2}}(\theta-\sin \theta) \\
1+\Delta(M, a) & =\frac{9}{2} \frac{(\theta-\sin \theta)^{2}}{(1-\cos \theta)^{3}}
\end{aligned}
$$

where $\theta=\theta(M, a)$. Replacing cosmic time for scale factor in equation (A5) $\left(a \propto t^{2 / 3}\right.$ for EdS), we can express the initial overdensity in terms of $a_{200}(M)$ :

$$
\Delta_{\mathrm{L}}\left(M, a_{i}\right)=\frac{3}{5} \frac{a_{i}}{a_{200}(M)}\left(\frac{3}{4}\left(\theta_{200}-\sin \theta_{200}\right)\right)^{2 / 3}
$$

where $\theta_{200} \simeq 4.8$ is the $\theta$ parameter for which $1+\Delta=200$ (i.e. the parameter at which the shell reaches $r=R_{200}$, and at which $\left.a=a_{200}\right)$. For a given shell at this point in its trajectory, its enclosed mass will now be identical to the total mass of the halo, so by equation (36)

$$
\begin{aligned}
& M=M_{200}=M_{\infty} e^{-s a_{f} / a_{200}(M)} \\
& a_{200}(M)=\frac{s a_{f}}{\ln \left(M_{\infty} / M\right)}
\end{aligned}
$$

Finally, substituting this into equation (A7), we obtain the exact form of equation (37):

$$
\begin{aligned}
\Delta_{\mathrm{L}}\left(M, a_{i}\right) & =\frac{3}{5} \frac{a_{i}}{s a_{f}}\left(\frac{3}{4}\left(\theta_{200}-\sin \theta_{200}\right)\right)^{2 / 3} \ln \left(M_{\infty} / M\right) \\
& \simeq 0.8 \frac{a_{i}}{a_{f}} \ln \left(M_{\infty} / M\right)
\end{aligned}
$$

\section{APPENDIX B: SETTING THE HORIZON-ENTRY SCALE FACTOR}

In our treatment of linear perturbations in the SFDM model, we use the thin-horizon approximation and define a "horizon-entry" scale factor that specifies when a mode transitions from superhorizon to subhorizon. A physically-motivated definition of horizon entry is roughly the scale factor at which the wavelength of a mode (with wavenumber $k$ ) equals the diameter of the Hubble sphere, so that:

$k=\frac{\pi}{c} a_{\mathrm{H}} H\left(a_{\mathrm{H}}\right)$

In the radiation-dominated era, this can be approximated by

$a_{\mathrm{H}} \approx \pi \frac{H_{0} \sqrt{\Omega_{\mathrm{rad}}}}{c k}$

However, in order to ensure that the perturbation amplitude is continuous across the horizon-entry transition, we must require that the horizon-entry scale factor be such that the equation describing the subhorizon evolution of the perturbation be equal to its superhorizon amplitude at the horizon-entry scale factor. For CDM perturbations, the subhorizon evolution is given by equation (72), so we define the

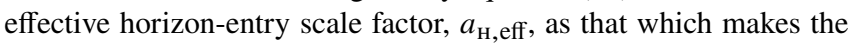
right side of this equation equal to 1 . In the radiation-dominated era, this can be approximated by

$a_{\mathrm{H}, \mathrm{eff}} \approx e^{2 / 3-\gamma_{\mathrm{E}} \sqrt{3} \frac{H_{0} \sqrt{\Omega_{\mathrm{rad}}}}{c k}} \simeq 2 \frac{H_{0} \sqrt{\Omega_{\mathrm{rad}}}}{c k}$

which is not far from the physically-motivated definition of $a_{\mathrm{H}}$. We compare both of these definitions as a function of $k$ in Fig. B1.

This paper has been typeset from a $\mathrm{T}_{\mathrm{E}} \mathrm{X} / \mathrm{LT} \mathrm{T} \mathrm{X}$ file prepared by the author. 


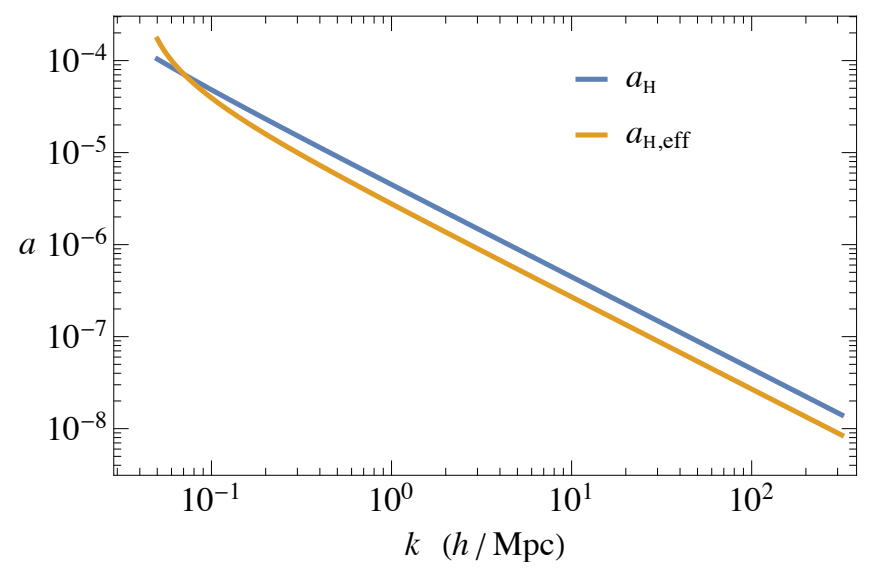

Figure B1. Comparison of the horizon-entry scale factors, $a_{\mathrm{H}}$ and $a_{\mathrm{H}, \mathrm{eff}}$, as defined by equations (B1) and (75), respectively. 